\title{
Burkina Faso
}

ATLAS POLITIQUE DE LA SÉCURITÉ ALIMENTAIRE ET NUTRITIONNELLE ET DE LA RÉSILIENCE

Wim Marivoet

John M. Ulimwengu

Leysa Maty Sall

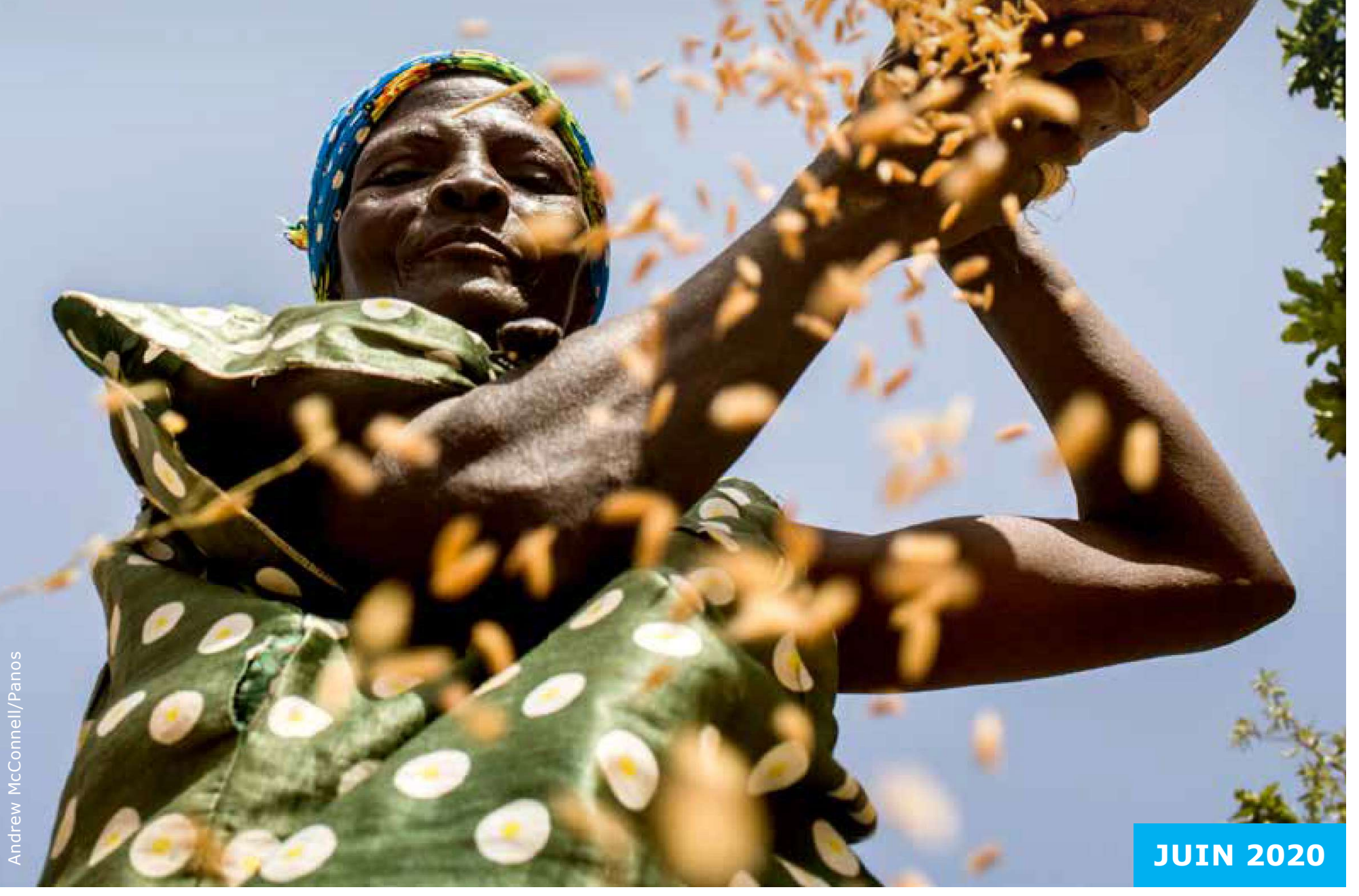




\section{A PROPOS DU PROGRAMME VOICE FOR CHANGE PARTNERSHIP (V4CP)}

Le partenariat «Voice for Change » (V4CP) est un programme multisectoriel financé par le ministère néerlandais des Affaires étrangères (DGIS), géré conjointement par l'Organisation néerlandaise de développement (SNV) et l'Institut international de recherche sur les politiques alimentaires (IFPRI) et mis en œuvre dans six pays : Burkina Faso, Ghana, Honduras, Indonésie, Kenya et Rwanda. Le Programme V4CP soutient les activités de plaidoyer des organisations de la société civile (OSC) afin d'influencer les politiques et les décisions. Grâce au renforcement de leurs capacités en matière de leadership, de développement des connaissances, de compétences de plaidoyer et de durabilité organisationnelle, les OSC sont habilitées à parler d'une voix plus forte. Le Programme V4CP forme les membres des OSC à l'utilisation des résultats de recherche, des données et des études de cas qu'ils peuvent ainsi utiliser pour renforcer leurs stratégies de plaidoyer. Le Programme V4CP aborde quatre questions - la sécurité alimentaire et nutritionnelle, la résilience, les énergies renouvelables et l'eau, I'assainissement et I'hygiène (WASH) tout en s'intéressant également au déséquilibre entre les genres et à l'atténuation du changement climatique. En collaborant avec des partenaires nationaux et internationaux, avec des entités à différents niveaux de gouvernement et avec le secteur privé, les OSC contribuent à établir un « environnement favorable ». Le renforcement de la voix des OSC dans l'élaboration des politiques et la mise en œuvre des services garantit une meilleure défense des intérêts des communautés qu'elles représentent.

\section{REMERCIEMENTS}

Ce rapport a été élaboré à partir de divers produits de connaissance et matériels de formation regroupés sous l'appellation « eAtlas imprimé », qui ont été développés et partagés avec les OSC dans le cadre du programme V4CP. Les auteurs sont reconnaissants aux membres de la SNV et des OSC pour les échanges fructueux et les commentaires constructifs formulés lors de plusieurs ateliers de renforcement des capacités et d'activités d'apprentissage. Il va de soi que toutes les erreurs demeurent sous la seule responsabilité des auteurs. 


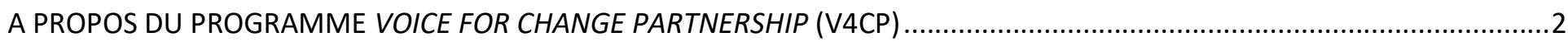

REMERCIEMENTS

Chapitre 1 : DES RESSOURCES À L'ÉLABORATION DES POLITIQUES : UN PARCOURS GÉOGRAPHIQUEMENT DÉSAGRÉGÉ ........5

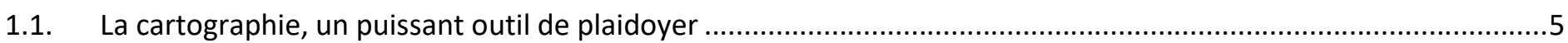

1.2. Les organisations de la société civile travaillant dans le cadre du Programme V4CP au Burkina Faso.....................5

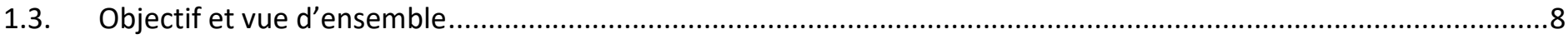

Chapitre 2 : TYPOLOGIE COMPLÈTE DES INTERVENTIONS DE SÉCURITÉ ALIMENTAIRE ET NUTRITIONNELLE .........................9

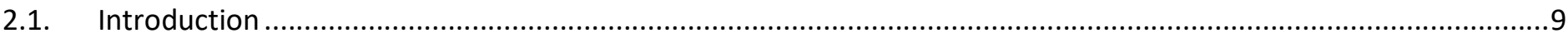

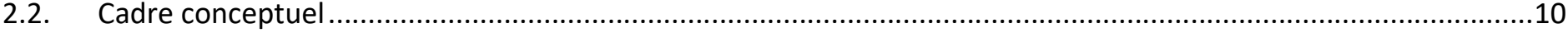

2.3. Les données sur la sécurité alimentaire et nutritionnelle .........................................................................12

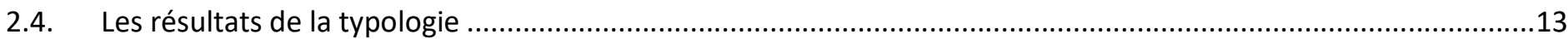

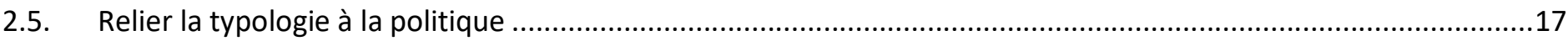

Chapitre 3 : CARTES D’ADÉQUATION DES NUTRIMENTS POUR DES INTERVENTIONS POLITIQUES CIBLÉES ...........................19

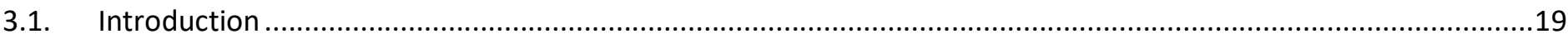

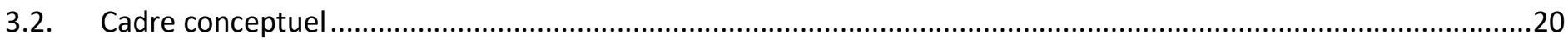

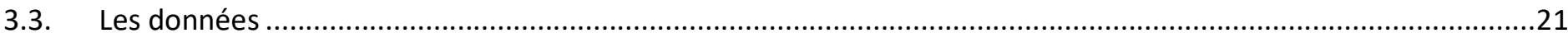

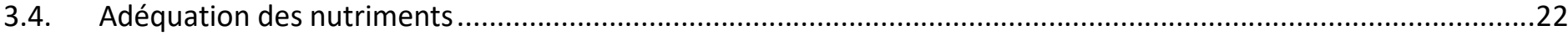

3.5. Retour aux produits alimentaires et politiques ciblées à partir des carences nutritionnelles..............................27

Chapitre 4 : CARTOGRAPHIE DE L'ADÉQUATION RELATIVE DES INFRASTRUCTURES D'ÉLEVAGE POUR UNE MEILLEURE

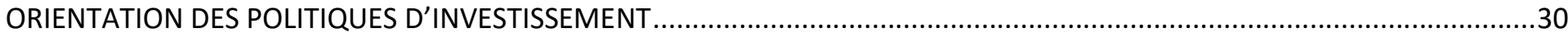

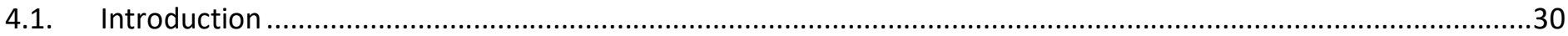

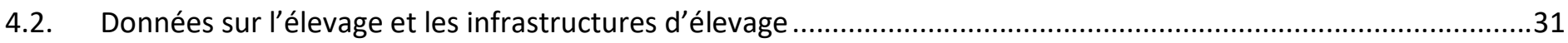

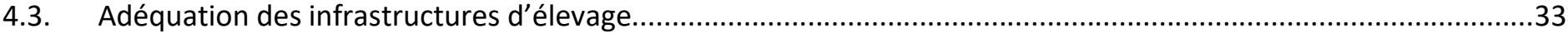

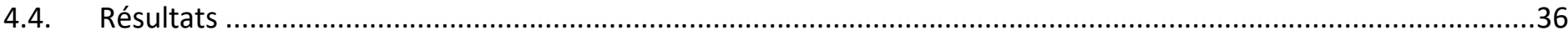

4.5. Vers une politique d'investissement plus ciblée sur le plan géographique .......................................................39

Chapitre 5 : LES OBSTACLES ET LES SOLUTIONS DE PLAIDOYER EN FONCTION DES ZONES GÉOGRAPHIQUES......................43

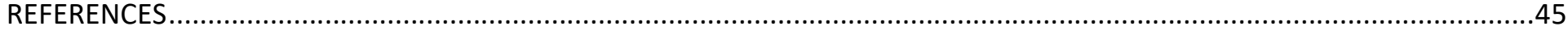




\section{Chapitre 1}

DES RESSOURCES À L'ÉLABORATION DES POLITIQUES : UN PARCOURS GÉOGRAPHIQUEMENT DÉSAGRÉGÉ

\subsection{LA CARTOGRAPHIE, UN PUISSANT OUTIL DE PLAIDOYER}

Un dicton populaire dit qu'un « bon croquis vaut mieux qu'un long discours ». En ce sens, les cartes sont des représentations de notre monde et de ses conditions. Elles illustrent les relations entre les conditions d'une région et celles des régions voisines. Les cartes peuvent exposer les frontières politiques, les routes, la population, l'état de santé, les conditions climatiques, les ressources naturelles, la pauvreté, les taux de scolarisation ou les activités économiques telles que la production agricole. Elles illustrent des informations concernant des emplacements géographiques, révèlent des schémas spatiaux et peuvent être utilisées pour comparer des schémas entre différentes caractéristiques physiques ou phénomènes sociaux.

Parce que les cartes nous aident à comprendre les relations spatiales entre différents phénomènes ou conditions, elles sont utiles pour comprendre où il convient de cibler notre attention et ainsi établir des priorités en conséquence. En effet, les cartes peuvent interagir avec divers types de données ; ce qui nous permet de concevoir des programmes plus ciblés et plus complets en illustrant les endroits où les besoins sont les plus importants, les obstacles qui entravent les progrès ainsi que les relations géographiques entre les différents défis. Les cartes peuvent être des outils puissants pour informer les décideurs politiques rapidement en mettant en évidence un problème particulier ainsi que les solutions concises et attrayantes pour le résoudre.

Les cartes de cet atlas politique utilisent des données et des statistiques sur l'agriculture, l'élevage et la nutrition pour concevoir des stratégies intégrant un point de vue géographique dans le but d'améliorer la sécurité alimentaire et nutritionnelle (SAN). Elles offrent une capture instantanée de réalités en constante évolution et donc susceptibles de changer lorsque des événements se produisent ou que des données plus précises deviennent disponibles. Pour obtenir les cartes les plus récentes, veuillez consulter I'eAtlas du Burkina Faso sur le site web du ReSAKSS (eatlas.resakss.org/Burkina-faso/fr) qui fournit également des données spatiales et des cartes supplémentaires en plus de celles utilisées pour ce rapport.

\section{Encadré 1.1. Les limites d’une approche spatiale}

Les représentations géographiques peuvent être trompeuses car les surfaces ne sont pas nécessairement proportionnelles aux chiffres de la population, surtout si l'on compare des zones vastes mais peu peuplées à de petites agglomérations urbaines denses. Les zones rurales sont généralement les zones les plus visibles sur les cartes, tandis que les centres urbains densément peuplés peuvent sembler insignifiants malgré la forte concentration de population. De même, les cartes n'affichent souvent que des moyennes concernant une unité administrative donnée, par exemple une province ou une région, ignorant ainsi la dispersion ou l'inégalité au sein de cette unité. Pour ces raisons, l'approche spatiale n'est qu'une méthode parmi d'autres permettant d'établir un niveau d'information approprié pour éclairer les décisions politiques.

\subsection{LES ORGANISATIONS DE LA SOCIETE CIVILE TRAVAILLANT DANS LE CADRE DU PROGRAMME V4CP AU BURKINA FASO}

Les OSC qui travaillent dans le cadre du Programme V4CP au Burkina Faso représentent les intérêts de divers groupes et communautés tels que les petits exploitants agricoles, les transformateurs de produits alimentaires, les éleveurs, les entrepreneurs, les partenaires au développement et les groupes environnementaux. En encourageant la collaboration interne et avec les parties prenantes concernées, les OSC influencent l'élaboration des programmes et mettent le gouvernement et le secteur privé devant leurs responsabilités en ce qui concerne leurs promesses et leurs actions. Le programme V4CP au Burkina Faso porte sur trois domaines thématiques : la sécurité alimentaire et nutritionnelle ; la résilience des communautés pastorales et ; les énergies renouvelables. Cet atlas politique fournit des données probantes qui soutiennent principalement le programme de plaidoyer des OSC travaillant sur les deux premiers domaines thématiques. Dans le cadre de la sécurité alimentaire et nutritionnelle, le principal objectif des OSC est de promouvoir la modernisation des exploitations agricoles familiales grâce à un meilleur accès aux intrants agricoles, aux équipements modernes et à la vulgarisation agricole. Les quatre OSC suivantes opèrent dans le cadre de ce domaine thématique : Coopérative de prestation de services agricoles Coobsa (COPSA-C) ; Union nationale des producteurs de riz du Burkina Faso (UNPRB) ; Fédération nationale des groupements Naam (FNGN) et Fédération des professionnels agricoles du Burkina (FEPA-B). Le principal programme de plaidoyer sous le thème de la résilience pastorale implique l'amélioration de la contex- 
tualisation locale des politiques d'élevage tout en répondant aux pressions du changement climatique et de la croissance démographique. Deux OSC se concentrent sur ce domaine particulier : Association pour la promotion de l'élevage dans le Sahel et la savane (APESS) et Plateforme d'actions à la sécurisation des ménages pastoraux (PASMEP).

L'encadré 1.2. fournit une brève description de chaque OSC avec leurs principales zones géographiques cibles. 


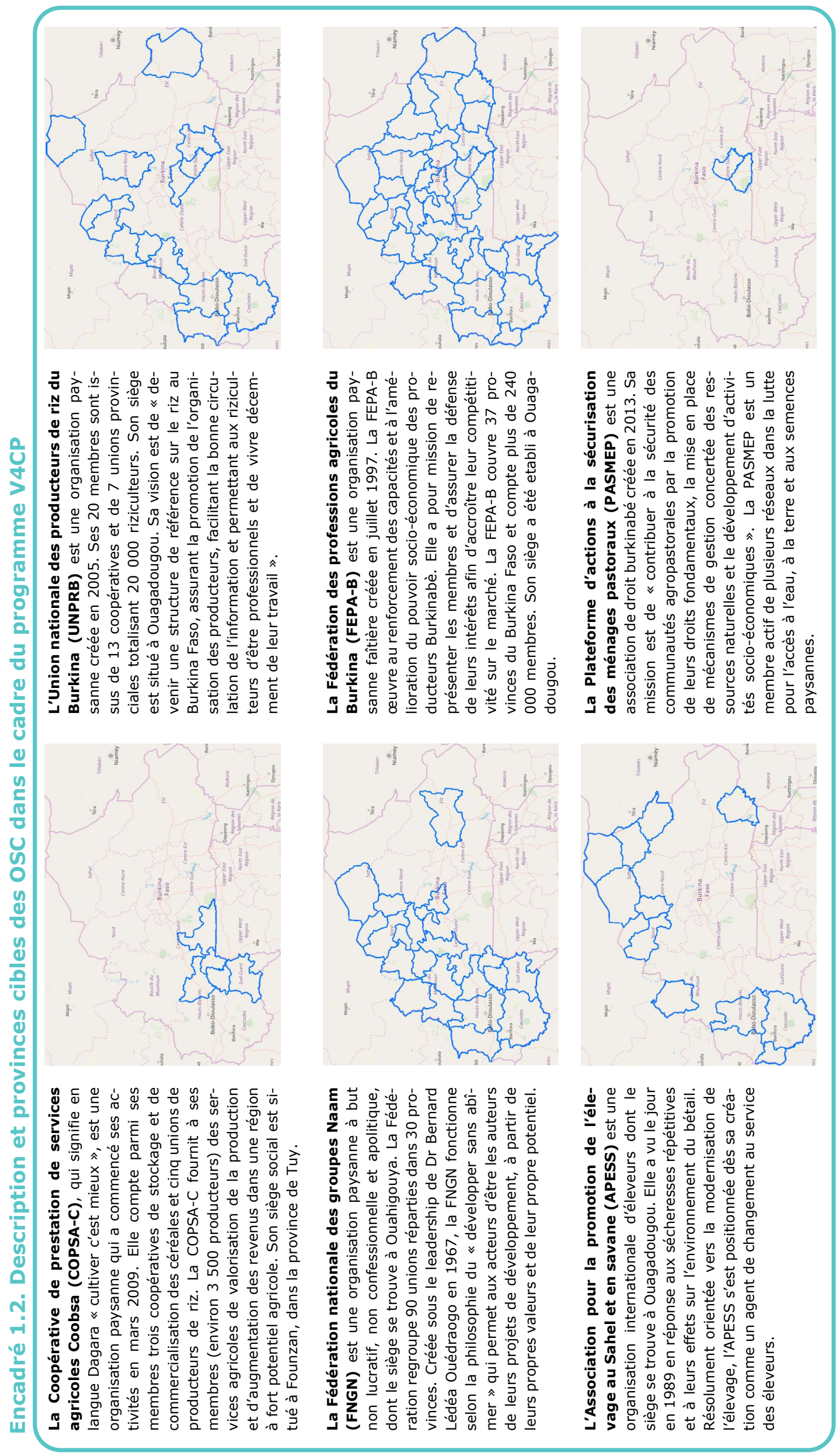




\subsection{OBJECTIF ET VUE D'ENSEMBLE}

Cet atlas politique utilise des cartes pour répondre à la question suivante : comment cibler plus efficacement les interventions en matière de nutrition, d'agriculture et d'élevage au Burkina Faso ? Les futures stratégies de plaidoyer des OSC pourraient être renforcées et enrichies par des informations spatiales indiquant les zones où les efforts politiques seraient les plus efficaces. Ce qui permettrait ainsi de s'attaquer aux obstacles auxquels sont confrontées les communautés les plus démunies, de réduire les disparités ou d'aider les zones à réaliser leur plein potentiel.

Le cadre conceptuel utilisé dans cet atlas politique est basé sur les piliers séquentiels de la sécurité alimentaire et nutritionnelle (SAN), généralement désignés par les termes de disponibilité, accès et utilisation. Pour que les populations disposent « d'un accès physique et économique à une nourriture suffisante, saine et nutritive leur permettant de satisfaire leurs besoins énergétiques et leurs préférences alimentaires pour mener une vie saine et active » (FAO, 1996), les contraintes liées à chaque pilier doivent être éliminées ou traitées de manière adéquate. La Figure 1.1. illustre la nature séquentielle ou hiérarchique de ces contraintes, qui ont toutes un impact sur les résultats nutritionnels finaux. Pour améliorer la nutrition, une population doit d'abord être en mesure de produire suffisamment de nourriture, ce qui dépend du niveau de potentiel agricole combiné aux contraintes de production auxquelles sont confrontés les agriculteurs. Alors que les contraintes biophysiques (telles que les précipitations, la température et les types de sol) déterminent le potentiel agricole, les contraintes de production font référence à toutes sortes d'intrants et de technologies agricoles sous-optimales (telles que des semences de qualité inférieure ou l'absence de vulgarisation agricole). En outre, les ménages devraient pouvoir acquérir des denrées alimentaires diverses, ce qui dépend de la quantité et de la variété des aliments produits ainsi que de diverses contraintes d'accès (telles que des infrastructures de transport de mauvaise qualité) ou des contraintes économiques (comme un pouvoir d'achat limité ou des restrictions commerciales). Une fois que les ménages ont acquis des produits alimentaires, ceux-ci doivent être correctement préparés et distribués à chaque membre de la famille, puis consommés dans des conditions particulières d'hygiène et de santé qui sont autant de contraintes d'utilisation.

Figure 1.1. Cadre général et schéma conceptuell de chaque chapitre

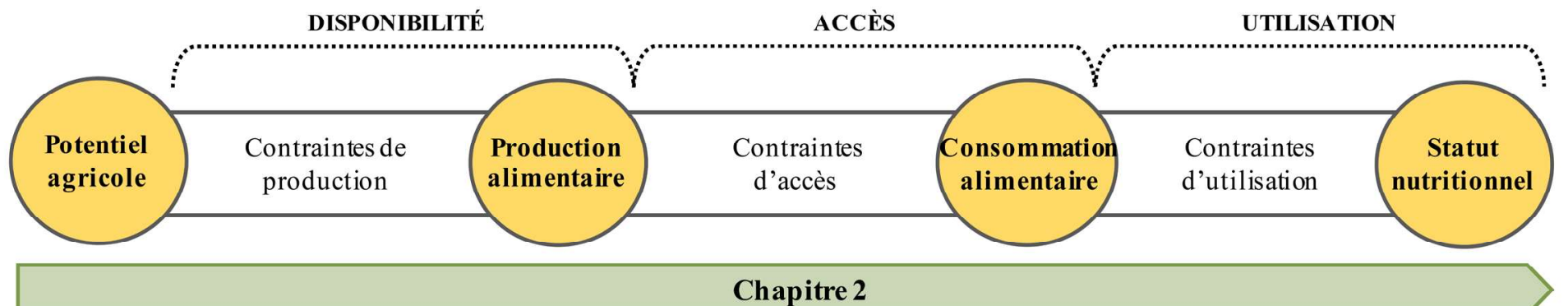

Chapitre 3

\section{Chapitre 4}

Source : Les Auteurs.

Chaque chapitre de cet atlas politique se concentre sur un schéma conceptuel différent à l'intérieur du cadre général. Le chapitre 2 évalue le degré de contrainte le long du chemin complet de la SAN pour chaque province du Burkina Faso en comparant les données réelles sur le potentiel agricole local, la production alimentaire, l'acquisition de denrées alimentaires et le statut nutritionnel. Le chapitre 3 explore les obstacles régionaux qui empêchent les ménages du Burkina Faso d’acquérir des produits alimentaires sains en quantité suffisante. En se concentrant sur les nutriments essentiels à la santé humaine, ce chapitre identifie les régions et les aliments à cibler pour améliorer la production agricole, l'intégration des marchés, la manipulation post-récolte ou l'allocation du budget alimentaire des ménages. Le chapitre 4 se concentre sur le sous-secteur de l'élevage, en donnant la priorité aux provinces qui sont sous-équipées en termes d'infrastructures d'élevage par rapport à la population locale de bétail. Le chapitre 5 présente un résumé des conclusions générales ainsi que des recommandations sur la manière dont ces résultats devraient orienter les décideurs politiques au Burkina Faso.

À la fin de chaque chapitre, nous fournissons une brève note de plaidoyer avec des messages clés indiquant comment mieux orienter les futures politiques grâce aux conclusions présentées. Tout au long de ce document, des encadrés explicatifs, des listes de mots clés et des infographies permettent de contextualiser chaque chapitre. 


\section{Chapitre 2}

\section{TYPOLOGIE COMPLÈTE DES INTERVENTIONS DE SÉCURITÉ ALIMENTAIRE ET NUTRITION-} NELLE

\subsection{INTRODUCTION}

Ce chapitre présente une typologie complète permettant d'identifier les interventions possibles en matière de sécurité alimentaire et nutritionnelle (SAN) dans les zones rurales du Burkina Faso. Nous commençons par identifier les provinces du Burkina Faso où les résultats nutritionnels sont les plus mauvais. Dans les sections suivantes, nous appliquons la typologie de manière à explorer les interventions susceptibles d'améliorer les résultats. Pour ce faire, nous comparons quatre indicateurs dans chaque province : le potentiel agricole, la production alimentaire, l'acquisition de produits alimentaires et les résultats en matière de nutrition. En comparant ces indicateurs, nous identifions les provinces qui bénéficieraient le plus d'une amélioration de l'efficacité en matière de production, d'accès ou d'utilisation. Ce chapitre se termine par des recommandations politiques et explique comment une analyse géographique spécifique peut guider la conception des interventions de SAN.

La Figure 2.1. présente la répartition spatiale de la malnutrition chronique chez les enfants de moins de 5 ans au Burkina Faso en 2010.

\section{Figure 2.1. Prévalence du retard de croissance chez les enfants de} moins de 5 ans dans les zones rurales du Burkina Faso (2010)

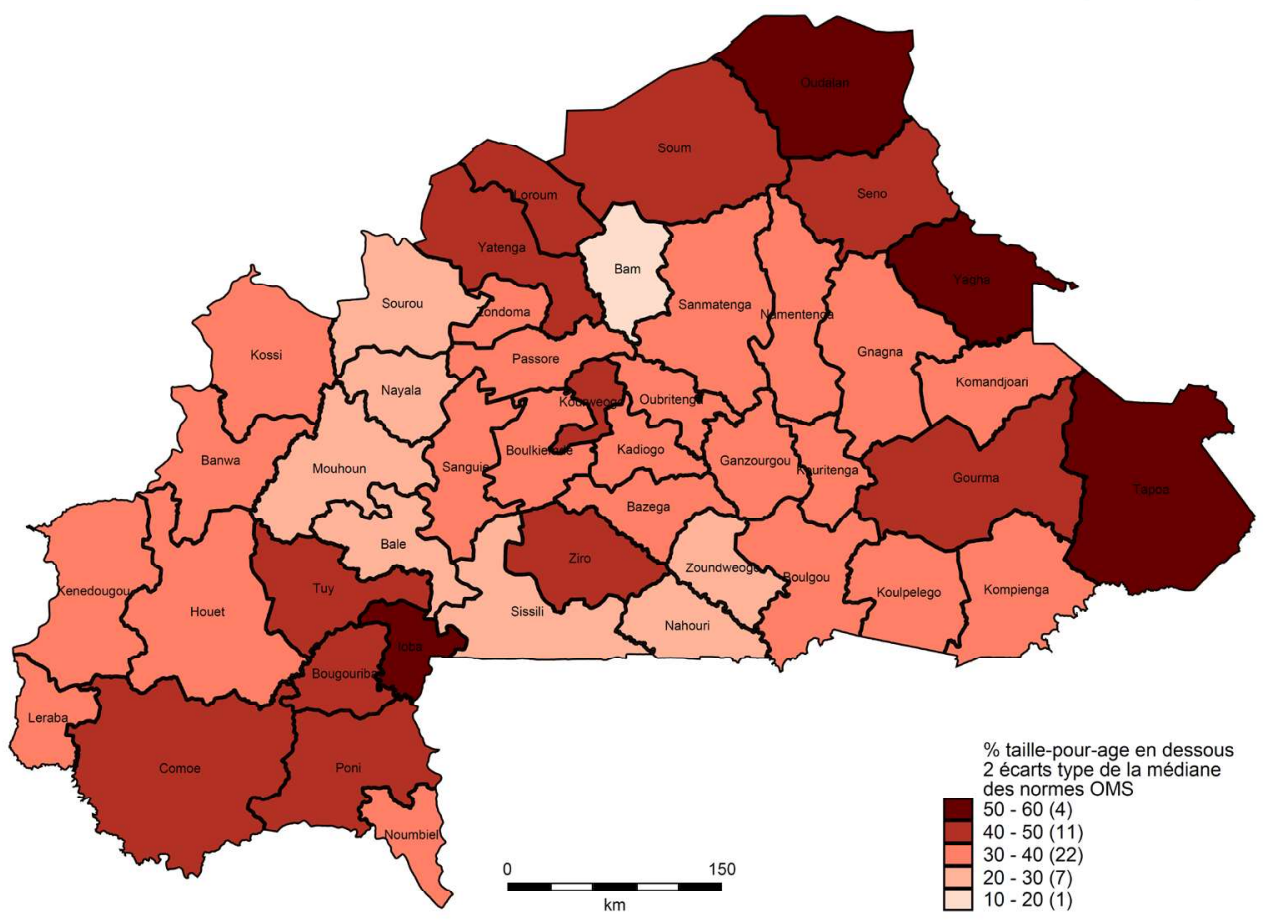

Source : Les Auteurs, à partir des données EDS (2010). ${ }^{1}$

\section{Mots clés}

Typologie : Réduction de la complexité du monde réel à des types ; classification.

Distribution spatiale : Etude des phénomènes et de leur localisation physique ; représentation graphique de ces informations.

Résolution spatiale : Nombre de pixels utilisés pour créer une image. Un nombre élevé de pixels (plus petits) offre une résolution et une définition plus élevées; et vice versa.

Télédetection : Scan de la terre à haute altitude (c.-à-d. par satellite) pour recueillir des informations.

Hétérogenéité spatiale : Répartition inégale d'une caractéristique à travers les régions.

Cadre conceptuel : Façon dont les idées sont organisées ; structure utilisée pour relier différents concepts et montrer leur orientation vers un objectif commun.

Coûts de transaction : Coûts associés à l'échange de biens/services dans le temps, aux lieus et aux marchés, mais distincts des coûts de production réels.

Indicateur : Unité mesurable qui sert de jauge pour indiquer l'état d'une chose.

\section{Diagramme de dispersion : Visualisa-}

tion de données qui montre la relation entre deux variables ; utilise des points pour représenter des valeurs numériques (une variable tracée sur l'axe des $x$ et l'autre sur l'axe des $y$ ).

Repère absolu : Basé sur une cible précise.

Repères relatifs : Comparent les performances des entités les unes par rapport aux autres.

\footnotetext{
${ }^{1}$ Bien que I'enquête SMART mise en œuvre en 2016 (ministère de la Santé du Burkina Faso, 2016) fournisse des taux de retard de croissance plus récents (et toujours plus faibles), sa précision spatiale est faible. Cependant, au niveau plus global, les deux sources de données s'alignent bien, classant de manière similaire les différentes régions en fonction de la prévalence de la malnutrition chronique. Cette comparaison relative entre les zones géographiques est l'objet principal de ce chapitre.
} 
La Figure 2.1. montre que la malnutrition chronique est plus élevée dans les zones les plus reculées du pays, en particulier dans les régions du Nord et de l'Est, et à l'extrême Sud-Ouest, où au moins $40 \%$ des enfants souffrent d'un retard de croissance. Ce n'est que dans la province de Bam que l'on observe des niveaux de retard de croissance inférieurs à $20 \%$. Dans un demi-cercle allant de Zoundweogo au Sud à Sourou au Nord, le retard de croissance se situe entre 20 et 30\%. Pour remédier au problème posé par les différents niveaux de statuts nutritionnels dans le pays, il est donc important de concevoir des interventions de politique qui tiennent compte de l'hétérogénéité spatiale.

\subsection{CADRE CONCEPTUEL}

Pour orienter les politiques et les programmes de lutte contre l'insécurité alimentaire et nutritionnelle dans tout le pays, il convient d'examiner le chemin conceptuel qui va du potentiel agricole au statut nutritionnel. Le cadre conceptuel présenté à la Figure 2.2. montre quatre dimensions de la SAN. Ces dimensions sont présentées de manière chronologique, chaque étape étant soumise à des contraintes. Par exemple, en ce qui concerne la production alimentaire, les agriculteurs doivent avoir accès à la terre, aux connaissances, au crédit, aux semences et aux engrais pour pouvoir exploiter le potentiel agricole de leurs terres. Plus en aval de la chaîne, même si la production alimentaire est suffisante, les familles peuvent être contraintes par toutes sortes de coûts de transaction tels que les barrières commerciales ou des infrastructures de transport défaillantes qui entraînent une hausse des prix locaux. Les résultats nutritionnels peuvent également être affectés par des contraintes d'utilisation telles que la question de la salubrité des aliments, les habitudes culinaires, les allocations intra-ménages ou encore les conditions sanitaires et d'hygiène.

Figure 2.2. Chemin conceptuel du potentiel agricole aux résultats nutritionnels
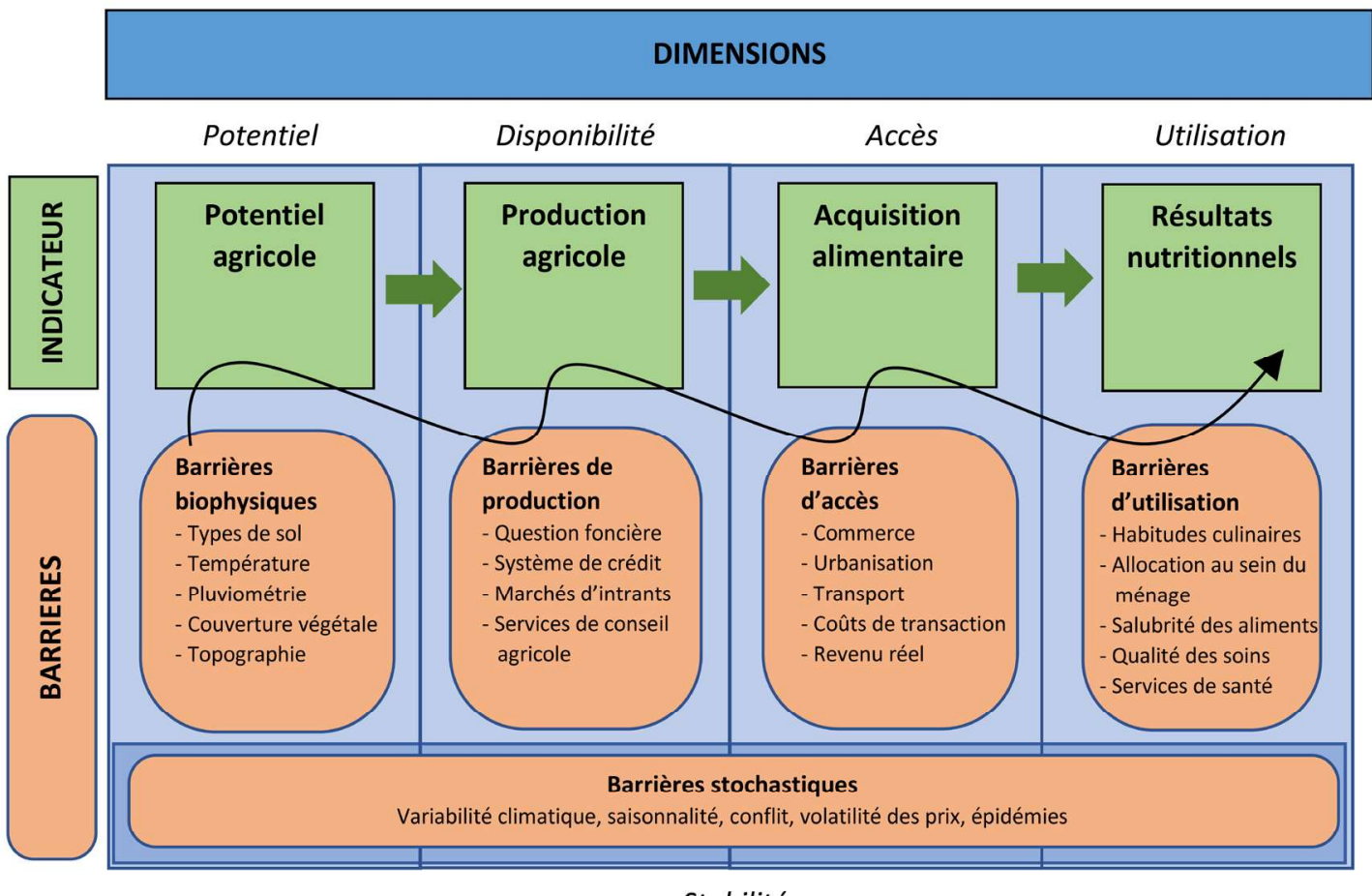

Stabilité

Source : Les Auteurs, adapté à partir de Pangaribowo et al. (2013).

En attribuant un indicateur à chacune des quatre dimensions séquentielles de la SAN (voir les encadrés verts de la Figure 2.2.), la typologie permet de déterminer la zone géographique la plus appropriée ainsi que le type d'intervention le plus efficace pour améliorer le statut nutritionnel de la population du Burkina Faso. La typologie classe chaque province en fonction de sa capacité à transformer :

(i) le potentiel agricole en production alimentaire (efficacité de production)

(ii) la production alimentaire en acquisition des produits alimentaires (efficacité d'accès)

(iii) l'acquisition de produits alimentaires en résultats nutritionnels (efficacité d'utilisation)

Pour estimer ces trois types d'efficacité, nous créons un diagramme qui relie tous les indicateurs. Le diagramme (voir Figure 2.3.) affiche chaque indicateur sur un axe différent, créant ainsi un diagramme en quatre dimensions. En partant de l'axe de mesure du potentiel agricole (axe vertical supérieur), ce diagramme doit être lu dans le sens des aiguilles d'une montre. Le panneau supérieur droit décrit la relation entre le potentiel agricole et la production alimentaire. Dans ce panneau, la ligne diagonale de l'efficacité moyenne indique le niveau de production alimentaire auquel on peut s'attendre en fonction du potentiel agricole de chaque zone. De même, la diagonale de l'efficacité d'accès (panneau inférieur droit) reflète les niveaux d'acquisition de produits alimentaires 
attendus en fonction du niveau de production alimentaire d'une province. Enfin, l'efficacité d'utilisation (panneau inférieur gauche) représente les niveaux attendus du statut nutritionnel pour chaque niveau d'acquisition de produits alimentaires. Ces trois types d'efficacité permettent de comparer les provinces du Burkina Faso entre elles, ce qui signifie que les niveaux attendus sont tous basés sur une comparaison relative avec les autres provinces.

En traçant une « fourchette » autour des lignes de rendement moyen, nous pouvons définir trois niveaux d'efficacité : supérieur à la moyenne, inférieur à la moyenne et moyen. Lorsque les données observées se situent à l'interieur de la «fourchette », l'efficacité est moyenne. Par exemple, pour les provinces (représentées par des points) qui se situent dans la « fourchette » de l'efficacité de production (panneau supérieur droit), le niveau de production alimentaire est à peu près celui auquel on pourrait s'attendre compte tenu de leur potentiel agricole (c'est-à-dire en fonction des principales contraintes de production moyennes qui caractérisent le pays). Cependant, lorsque les données observées se situent en dehors de cette « fourchette », les performances de la production alimentaire sont soit pires soit meilleures que ce qui est (en moyenne) observé dans tout le pays. Ainsi, nous classons les provinces qui obtiennent des résultats supérieurs à $125 \%$ de la moyenne du pays comme étant « au-dessus de la moyenne » (résultats représentés par l'espace blanc dans les quadrants de la Figure 2.3.). Nous classons les provinces qui obtiennent des résultats inférieurs à $75 \%$ de la moyenne nationale comme étant « en-dessous de la moyenne » (espace gris foncé) ; et les provinces « moyennes » se situent dans la « fourchette » 75-125\% (espace gris clair). Le même étalonnage relatif des performances «au-dessus de la moyenne», « en-dessous de la moyenne » et «moyen » s'applique à l'efficacité d'accès (panneau inférieur droit) et à l'efficacité d'utilisation (panneau inférieur gauche).

\section{Figure 2.3. Diagramme combiné des dimensions de la SAN}

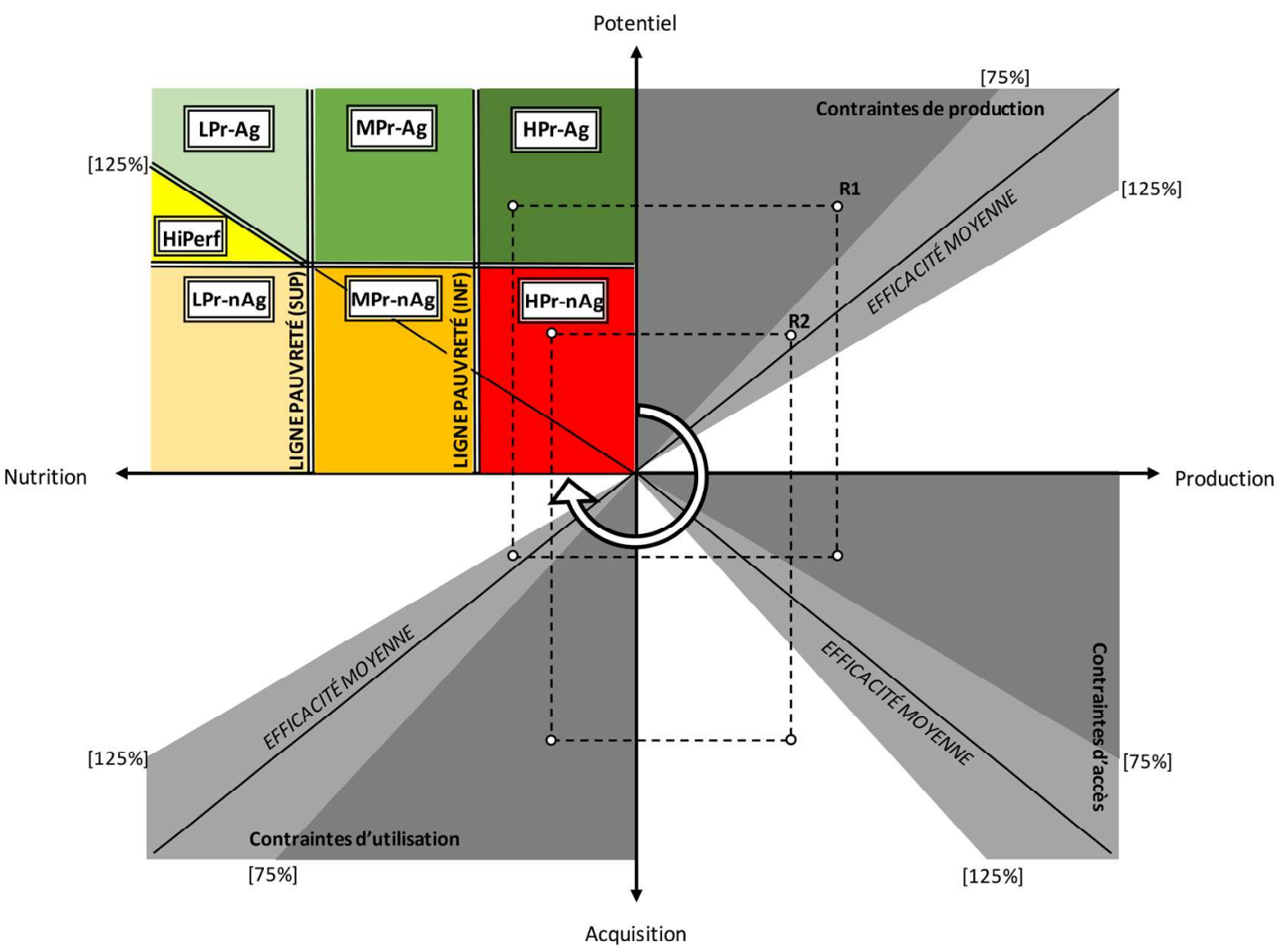

Notes : LPr, MPr, HPr, respectivement pour les provinces à faible, moyenne et haute priorité nutritionnelle ; Ag et nAg désignent un potentiel agricole plus élevé $(\mathrm{Ag})$ et plus faible $(\mathrm{nAg})$; HiPerf désigne les provinces à haute performance. Les lignes de pauvreté « (inf) 》 et «(sup) 》 désignent les seuils de pauvreté inférieur et supérieur.

Source : Les Auteurs.

Le panneau supérieur gauche de la Figure 2.3. montre les seuils de pauvreté pour les zones à faible, moyenne et haute priorité nutritionnelle. En outre, le panneau supérieur gauche fait la distinction entre les zones présentant de grandes opportunités agricoles et les zones présentant de faibles opportunités agricoles. ${ }^{2}$ Sur la base de ces définitions, on distingue sept types d'intervention, qui résultent de la combinaison des trois niveaux de priorité - zone de haute priorité (HPr), zone de moyenne priorité (MPr)

\footnotetext{
${ }^{2}$ Cette distinction est basée sur le niveau du potentiel agricole qui correspond à l'intersection du seuil de pauvreté supérieur et de la ligne représentant $125 \%$ de l'efficacité moyenne entre le potentiel et la nutrition. Cela signifie que les zones dont le potentiel agricole est inférieur à ce seuil n'atteindront pas un statut nutritionnel supérieur au seuil de pauvreté supérieur, à moins qu'elles n'obtiennent des résultats supérieurs à $125 \%$ de ce qui est, en moyenne, observé dans le pays.
} 
et zone de faible priorité ( $\mathrm{LPr}$ ) - et de deux niveaux de potentiel agricole - un potentiel agricole supérieur ( $\mathrm{Ag}$ ) ou un potentiel agricole inférieur $(\mathrm{nAg}) .^{3}$ Dans la catégorie des «zones à faible priorité et à potentiel agricole élevé $(\mathrm{LPr}-\mathrm{Ag})$ 》, on peut également classer les zones comme « hautement performantes (HiPerf) » lorsque leur niveau d'efficacité global est supérieur à $125 \%$ de la moyenne du pays. Ces zones pourraient servir d'exemples pour d'autres régions et ne pas se prêter à une intervention de SAN car elles sont déjà performantes en matière de conversion du potentiel agricole en résultats nutritionnels.

En combinant toutes les informations, le diagramme en quatre dimensions qui se trouve sous cette typologie décrit deux sortes d'étalonnage :

1) Le panneau supérieur gauche applique un repère absolu pour montrer le niveau d'urgence en matière de nutritionpauvreté. Ce panneau permet également de déterminer s'il est justifié de se concentrer sur la production agricole ;

2) Les trois autres panneaux appliquent un repère relatif en comparant la performance de chaque province avec les résultats attendus tout en donnant des détails approximatifs sur l'endroit - sur le chemin qui va du potentiel agricole au statut nutritionnel - où les gains les plus importants peuvent être réalisés en termes de réduction des contraintes de production, d'accès ou d'utilisation.

\section{Encadré 2.1. Exemples de typologie}

Cet encadré présente le profil typologique de deux régions fictives, R1 et R2, en capturant des données observées à partir de diverses dimensions dans la Figure 2.3. Chaque région est représentée par quatre points de données, en fonction de ses valeurs dans chacune des quatre dimensions. L'emplacement précis des points de données à l'intérieur de chacun des panneaux caractérise donc la région. Le panneau supérieur gauche définit le niveau de priorité, les couleurs plus foncées (vert ou rouge) indiquant une plus grande urgence, tandis que les autres panneaux donnent un aperçu du type d'intervention qui serait le plus efficace pour améliorer les résultats en matière de nutrition.

R1

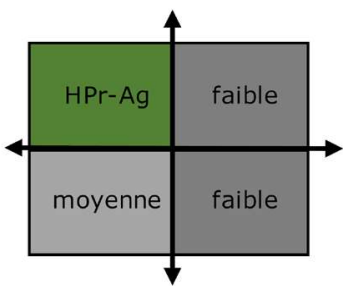

R2

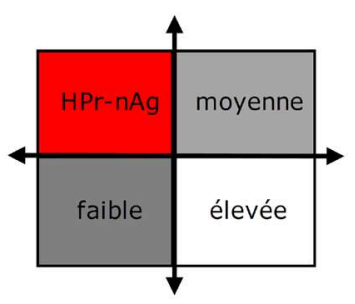

R1 est une « région hautement prioritaire à potentiel agricole élevé ( $\mathrm{HPr}-\mathrm{Ag}$ ) 》 parce que le statut nutritionnel de sa population est faible alors que le potentiel agricole est élevé. Pour améliorer les résultats en matière de nutrition dans cette région, il convient d'accorder plus d'attention à la suppression des contraintes de production et d'accès, car l'efficacité dans ces deux dimensions est inférieure à ce qui est (en moyenne) observé dans l'ensemble du pays. L'efficacité d'utilisation est moyenne et donc considérée comme un obstacle moins critique.

$\mathrm{R} 2$ est une « région hautement prioritaire à faible potentiel agricole ( $\mathrm{HPr}-\mathrm{nAg}$ ) » parce que le statut nutritionnel de sa population ainsi que son potentiel agricole sont faibles. Contrairement à $\mathrm{R} 1$, nous ne pouvons pas nous attendre à des améliorations nutritionnelles majeures résultant de la suppression des contraintes de production parce que le potentiel agricole de la région est faible et son efficacité de production est déjà moyenne. Nous ne pouvons pas non plus nous attendre à des avantages nutritionnels majeurs du fait de l'amélioration de l'accès à la nourriture, parce que l'efficacité d'accès de la région est déjà supérieure à la moyenne du pays. Pour améliorer les résultats nutritionnels, R2 devrait principalement se concentrer sur la réduction des contraintes d'utilisation, étant donné que l'efficacité de cette dimension est faible.

\subsection{LES DONNEES SUR LA SECURITE ALIMENTAIRE ET NUTRITIONNELLE}

Sur la base du cadre conceptuel ci-dessus et en s'appuyant sur diverses sources de données, nous construisons un indicateur clé pour chaque dimension de la SAN. ${ }^{4}$ Pour faciliter la lecture, le Tableau 2.1. résume les indicateurs pour chaque dimension et fournit quelques statistiques descriptives.

Pour estimer le potentiel agricole de chaque province du Burkina Faso, nous considérons le nombre de kilocalories qui pourraient être produites à partir de toutes les terres arables disponibles, ce qui correspond essentiellement à la conversion d'hectares en équivalent kilocalories. Pour l'estimation des terres agricoles, nous nous appuyons sur deux sources de données de télédétection par satellite. La première source identifie toutes les terres utilisées pour l'agriculture en 2015, avec une résolution spatiale de $30 \mathrm{~m}$ pixels (Xiong et al., 2017). La seconde source (Hansen et al., 2013) indique la quantité de forêt défrichée entre 2000 et 2015, en supposant que ces terres ont été défrichées pour l'agriculture (Ouedraogo et al., 2010). Nous utilisons ces données satellites pour créer une carte des terres (immédiatement) arables dans tout le pays. Nous attribuons à chaque pixel de terre arable une

\footnotetext{
${ }^{3}$ Ces types s'inspirent en grande partie du travail de Torero (2014).

${ }^{4}$ Nous utilisons des indicateurs de données de différentes périodes, sur la base de la disponibilité la plus récente ; nous supposons que la structure géographique des données plus anciennes reste constante dans le temps.
} 
culture (maïs, mil, riz, sorgho rouge et sorgho blanc) proportionnelle à sa part dans la consommation nationale globale. Nous avons obtenu les données du «panier alimentaire » national auprès du Ministère de l'Agriculture du Burkina Faso (2010). En utilisant les données sur le rendement optimal (qui suppose une fertilisation adéquate, de bonnes pratiques agronomiques et de bonnes conditions pluviales) de l'Atlas mondial des écarts de rendement pour le Burkina Faso, chaque pixel de terre arable attribué à la culture correspondante est ensuite converti pour estimer la production agricole et les kilocalories correspondantes. ${ }^{5,6}$ Pour chaque province, nous additionnons la production potentielle totale de kilocalories de tous les pixels avant de la diviser par le nombre de personnes vivant dans cette province, ce qui nous donne alors les kilocalories potentiellement disponibles par personne. ${ }^{7}$

Pour calculer un indicateur de la production alimentaire, nous nous appuyons sur les statistiques provinciales de la production agricole réelle en 2015/2016, obtenues auprès du Ministère de l'Agriculture du Burkina Faso (2016). Nous convertissons ces statistiques de production 2015/2016 concernant les céréales, les tubercules et les légumineuses les plus importants en kilocalories en utilisant les mêmes données de composition alimentaire que ci-dessus (Stadlmayr et al., 2012). De nouveau, pour chaque province, nous divisons ensuite la production totale de kilocalories par la population de cette province, ce qui donne le nombre réel de kilocalories disponibles par personne au niveau provincial. En raison des données limitées, nous excluons la production agricole de source animale, telle que la viande, le poisson, le lait et les œufs. Pour représenter l'acquisition de produits alimentaires, nous utilisons le score de consommation alimentaire (SCA) du PAM dérivé de l'analyse globale de la sécurité alimentaire et de la vulnérabilité (CFSVA) de 2012. Basé sur les données de rappel de la consommation des groupes de produits alimentaires au cours des 7 derniers jours, ce score composite prend en compte la diversité alimentaire, la fréquence de consommation et l'importance nutritionnelle relative des différents groupes de produits alimentaires (WFP, 2008). Pour chaque province, nous calculons le pourcentage de ménages ayant un score acceptable (c'est-à-dire supérieur à 35,5, selon les recommandations du Programme alimentaire mondial). Pour la nutrition, nous nous appuyons sur les données de l'enquête démographique et de santé (EDS) de 2010. Nous définissons notre indicateur de nutrition comme le pourcentage d'enfants de moins de cinq ans qui ne présentent pas de retard de croissance (c'est-à-dire dont le rapport taille/âge est supérieur au seuil de malnutrition de la population de référence).

Tableau 2.1. Statistiques descriptives des indicateur's clés de la SAN dans les zones rurales du Burkina Faso (2010-2015)

\begin{tabular}{|c|c|c|c|c|c|}
\hline Indicateur & Composantes de I'indicateur & Obs. & Moyenne & Min & Max \\
\hline \multirow[t]{3}{*}{ Potentiel } & Terres immédiatement arables $\left(\mathrm{km}^{2}\right)$ & 45 & 1490,2 & 13,0 & 4994,0 \\
\hline & Production potentielle quotidienne de kilocalories par personne & 45 & 12030,8 & 86,5 & 33274,4 \\
\hline & $\begin{array}{l}\text { Transformation racine carrée de la production potentielle quotidienne } \\
\text { de kilocalories par personne }\end{array}$ & 45 & 101,2 & 9,3 & 182,4 \\
\hline \multirow[t]{2}{*}{ Production } & Production quotidienne de kilocalories par personne & 45 & 4421,9 & 1745,8 & 11198,3 \\
\hline & $\begin{array}{l}\text { Transformation racine carrée de la production quotidienne de kilocalo- } \\
\text { ries par personne }\end{array}$ & 45 & 64,5 & 41,8 & 105,8 \\
\hline \multirow[t]{2}{*}{ Acquisition } & $\%$ de ménages ayant un SCA inférieur à 35,5 & 45 & 30,8 & 2,8 & 90,3 \\
\hline & $\%$ de ménages ayant un SCA supérieur à 35,5 & 45 & 69,2 & 9,7 & 97,2 \\
\hline \multirow[t]{2}{*}{ Nutrition } & $\begin{array}{l}\text { \% d'enfants souffrant d'un retard de croissance ( }<5 \text { ans, avec un } \\
\text { écart-type inférieur à }-2 \text { par rapport à la médiane de la taille pour âge } \\
\text { de la population de référence) }\end{array}$ & 45 & 37,4 & 12,8 & 51,4 \\
\hline & $\begin{array}{l}\text { \% d'enfants ne souffrant pas d'un retard de croissance ( }<5 \text { ans, avec } \\
\text { un écart-type supérieur à }-2 \text { par rapport à la médiane de la taille pour } \\
\text { âge de la population de référence) }\end{array}$ & 45 & 62,6 & 48,6 & 87,2 \\
\hline
\end{tabular}

Source : Les Auteurs, avec des données provenant de Brown de Colstoun et al. (2017); CFSVA (2012); DHS (2010); Hansen et al. (2013); Ministère de I'Agriculture du Burkina Faso (2010, 2016); Pekel et al. (2016); Stadlmayr et al. (2012); UNEP-WCMC (2018); Xiong et al. (2017).

\subsection{LES RESULTATS DE LA TYPOLOGIE}

Après avoir défini et estimé les indicateurs pour chaque dimension de la SAN dans chacune des 45 provinces rurales du Burkina Faso, la Figure 2.4. présente le diagramme en quatre dimensions chargé de données réelles. Ce diagramme se compose de quatre nuages de points dans lesquels chaque point représente une province et ses deux valeurs correspondant aux deux indicateurs constitutifs. La Figure 2.5. ajoute la dimension spatiale en présentant quatre cartes du pays, chacune couvrant un quadrant des Figures 2.3. et 2.4., en indiquant le niveau d'efficacité de production, d'accès ou d'utilisation et une avec des couleurs représentant

\footnotetext{
${ }^{5}$ Voir http://www.yieldgap.org/burkina-faso.

${ }^{6}$ Basé sur les données de composition des aliments compilées par Stadlmayr et al. (2012).

7 Pour observer plus facilement les différences entre les provinces, nous appliquons une transformation racine carrée sur la production potentielle totale de kilocalories. Pour plus de cohérence, cette procédure est ensuite répétée pour notre mesure de la production réelle de kilocalories.
} 
les types d'intervention. Pour les trois types d'efficacité (panneau b, c et d), la couleur grise la plus claire indique les provinces dont l'efficacité est « au-dessus de la moyenne », tandis que la couleur noire désigne les provinces caractérisées par une efficacité « en-dessous de la moyenne ». Ces dernières provinces seraient des candidates potentielles pour des interventions ciblées visant à améliorer l'efficacité de la production, de l'accès ou de l'utilisation. Le panneau (a) combine les niveaux de priorité nutritionnelle des provinces avec le potentiel agricole relatif de ces provinces, en affichant les sept types d'intervention.

Nous observons 15 provinces hautement prioritaires, cinq à faible potentiel agricole et dix à fort potentiel agricole. Les provinces de Oudalan, Soum, Loroum, Yatenga, dans le Nord du pays exposé à la sécheresse, et la province de Kourweogo, située au centre du pays, ont un potentiel agricole moindre. Les provinces hautement prioritaires à potentiel agricole plus élevé sont dispersées dans tout le pays : Seno, Yagha, Tapoa et Gourma à l'Est, à la frontière du Niger, Ziro au centre, et un groupe de cinq autres provinces (Tuy, Ioba, Bougouriba, Comoe et Poni) dans le Sud-Ouest du pays.

Bien que ces provinces aient toutes besoin d'interventions nutritionnelles urgentes (en fonction des niveaux de retard de croissance chez les enfants), le type d'intervention optimale dépendra de leur emplacement. Les quatre provinces du Nord présentent des combinaisons différentes de contraintes de production et d'accès, mais en termes d'utilisation, leurs résultats sont inférieurs à $75 \%$ de la moyenne attendue dans les zones rurales du Burkina Faso. De même, les contraintes d'utilisation sont tout aussi difficiles à surmonter dans de nombreuses autres provinces hautement prioritaires, souvent combinées à de graves inefficacités de production dans les provinces de l'Est et à Comoé, et des contraintes d'accès à Bougouriba et à Ziro. Kourweogo dans le centre et de nombreuses provinces autour de Ouagadougou présentent des contraintes de production et d'utilisation plutôt modestes mais souffrent de graves problèmes d'accès aux produits alimentaires. Pour les provinces autour de Ouagadougou, cela pourrait être dû au fait que, malgré un bon accès aux intrants de production et aux infrastructures sanitaires dans la capitale, la forte demande de denrées alimentaires de la part de la population croissante de la région se traduit par des prix alimentaires plus élevés et une alimentation moins diversifiée. Nous reviendrons sur le thème du faible accès à une alimentation diversifiée dans le chapitre 3. 
Figure 2.4. Nuage de points combiné avec des données provinciales, zones rurales du Burkina Faso (2010-2015)

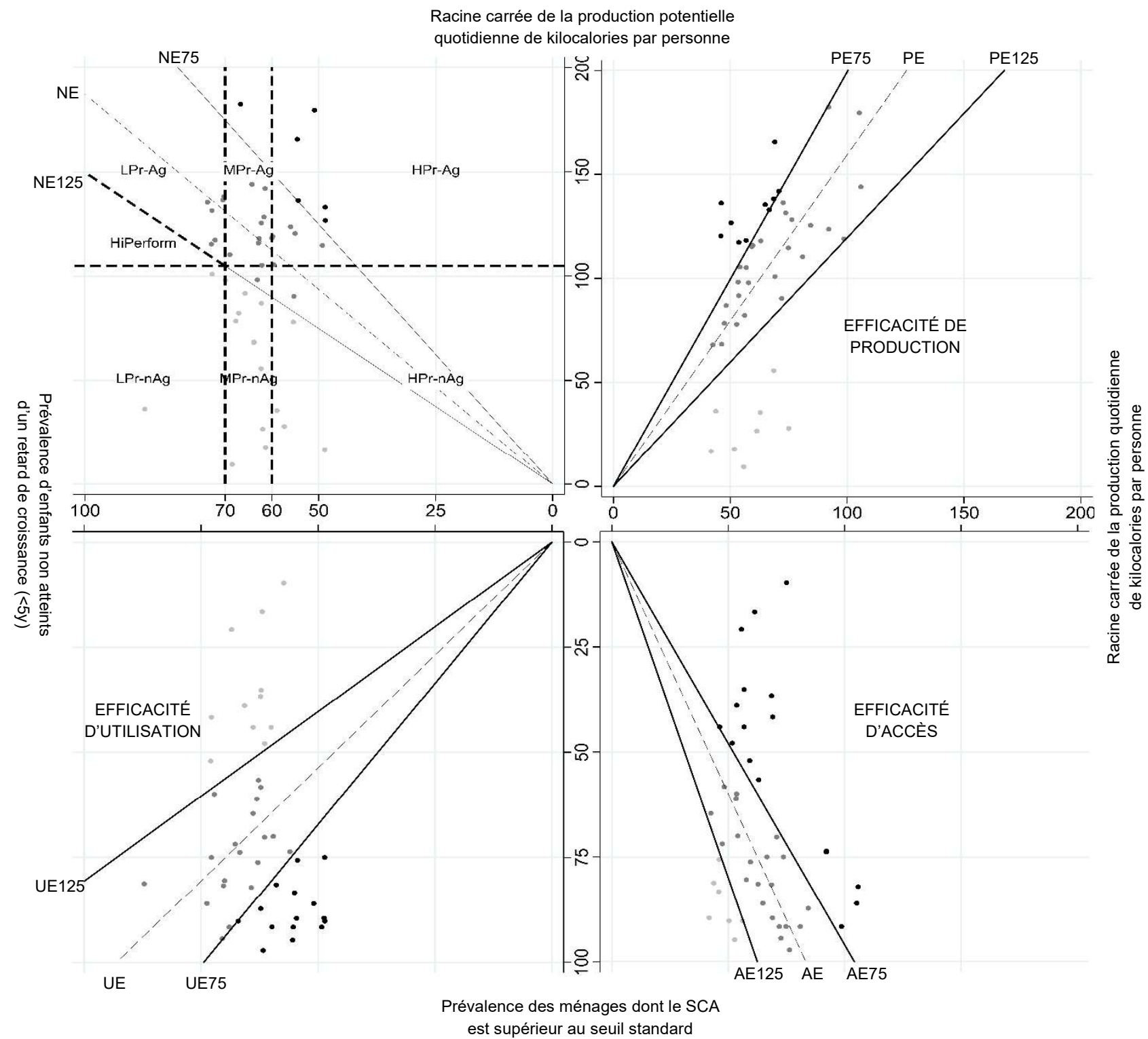

Notes : LPr, MPr, HPr désignent respectivement les provinces à faible, moyenne et haute priorité nutritionnelle ; Ag et nAg désignent un potentiel agricole plus élevé $(\mathrm{Ag})$ et plus faible $(\mathrm{nAg})$; HiPerform désigne les provinces à haute performance. $\mathrm{PE}, \mathrm{AE}$, UE et NE sont des lignes estimées basées sur des régressions MCO pondérées par la population avec interception par l'origine, ayant respectivement une pente de 1,594, 0,884, 0,985 et 0,534. Les lignes E75 et E125 sont dérivées des lignes précédentes avec des pentes de $75 \%$ et $125 \%$ de la taille des pentes estimées.

Source : Les Auteurs, à partir des données de Brown de Colstoun et al. (2017); CFSVA (2012); DHS (2010); Hansen et al. (2013); Ministère de I'Agriculture du Burkina Faso (2010, 2016); Pekel et al. (2016); Stadlmayr et al. (2012); UNEP-WCMC (2018); Xiong et al. (2017). 

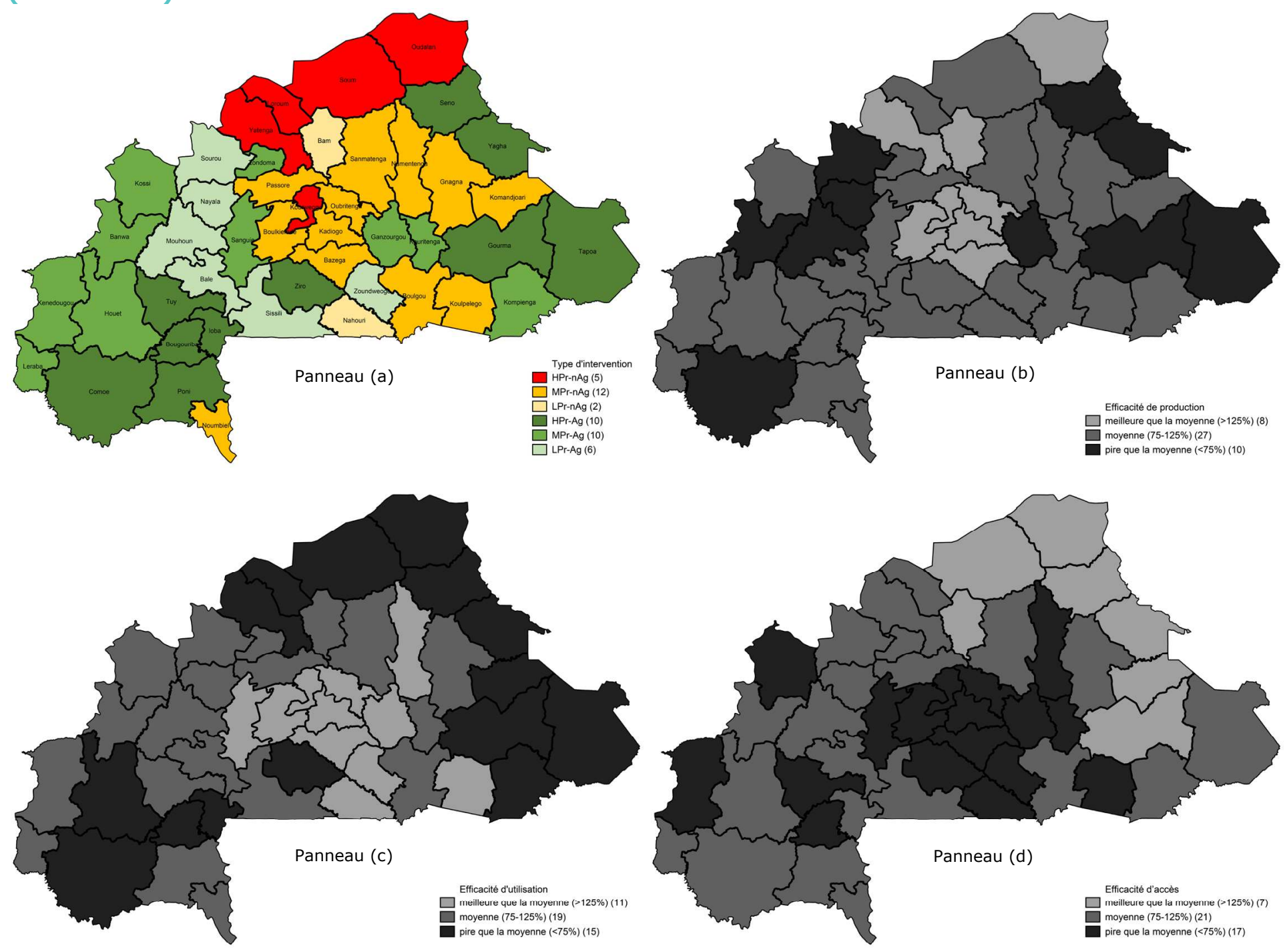

Notes : $\mathrm{LPr}, \mathrm{MPr}$, HPr désignent respectivement les provinces à faible, moyenne et haute priorité ; Ag et nAg désignent un potentiel agricole plus élevé (Ag) et plus faible (nAg).

Source : Les Auteurs, à partir des données de Brown de Colstoun et al. (2017); CFSVA (2012); DHS (2010); Hansen et al. (2013); Ministère de l'Agriculture du Burkina Faso (2010, 2016); Pekel et al. (2016); Stadlmayr et al. (2012); UNEP-WCMC (2018); Xiong et al. (2017). 


\subsection{RELIER LA TYPOLOGIE A LA POLITIQUE}

Le deuxième Programme national du secteur rural (PNSR2) du Burkina Faso (SP/CPSA, 2017) vise à améliorer le bien-être des populations rurales du Burkina Faso en ciblant six objectifs : (1) les systèmes de production inefficaces et les faibles taux de croissance agricole ; (2) la faiblesse des chaînes de valeur et des marchés agricoles ; (3) la fragilité de la sécurité alimentaire et nutritionnelle ; (4) la dégradation des ressources naturelles et la gestion durable ; (5) la mobilisation des ressources en eau et (6) le manque de capital humain et de supervision dans les zones rurales. En alignant les investissements sur les défis et les opportunités propres à chaque province, le pays serait mieux à même d'atteindre les six objectifs. Les interventions stratégiques de la SAN pourraient se concentrer sur les 15 provinces hautement prioritaires et un plan d'action adapté au niveau local pourrait être conçu pour chacune d'entre elles en fonction de la combinaison particulière des contraintes observées, comme le résume le Tableau 2.2 .

Par exemple, divers sous-programmes sous les axes 1 (production alimentaire), 4 (mobilisation de l'eau) et 5 (vulgarisation) visent à améliorer la productivité agricole, mais ne bénéficient pas d'une orientation spatiale. Ces programmes pourraient être plus utilement ciblés sur les provinces hautement prioritaires de Gourma, Seno, Tapoa, Yagha et Comoe. Les investissements liés à I'accès au marché et aux chaînes de valeur proposés sous l'axe 2 pourraient cibler explicitement les provinces de Kourweogo, Tuy, Bougouriba et Ziro, qui souffrent le plus des inefficacités du marché. Dans ces provinces, les relations entre la production, la transformation et les consommateurs pourraient être améliorés. Pour répondre aux problèmes d'utilisation dans les provinces de Gourma, Seno, Tapoa, Yagha, Comoe, Bougouriba, Ziro, Loroum, Yatenga, Oudalan, Soum et Ioba, I'axe 4 (eau et assainissement) du PNSR2 pourrait cibler ces zones géographiques où l'accès à une eau et un assainissement améliorés fait défaut. Bien que la stratégie de développement agricole et les plans d'investissement actuels du Burkina Faso pourraient être améliorés par un ciblage spatial, le Tableau 2.2. présente quelques cas dans lesquels les investissements correspondent bien aux profils d'inefficacité de certaines provinces. Tapoa, par exemple, qui souffre d'une faible efficacité de production, pourrait bénéficier de la construction d'une unité d'engrais dans le cadre du PNSR2. En revanche, Sourou et Houet reçoivent une part disproportionnée du financement global des investissements, alors que le taux de malnutrition de ces provinces n'est pas critique. Toutefois, le développement agricole de Samendeni (Houet) et de Sourou peut se justifier si des opportunités économiques suffisantes se multiplient dans les provinces prioritaires environnantes.

Tableau 2.2. Profill d'efficacité des provinces hautement prioritaires dans les zones rurales du Burkina Faso (2010-2015) par rapport au PNSR2

\begin{tabular}{|c|c|c|c|c|c|}
\hline Province & $\begin{array}{l}\text { Potentiel } \\
\text { agricole }\end{array}$ & Production & $\begin{array}{l}\text { Efficacité } \\
\text { Accès }\end{array}$ & Utilisation & $\begin{array}{l}\text { Second Programme National du Secteur Rural } \\
\text { (PNSR2) }\end{array}$ \\
\hline $\begin{array}{l}\text { Gourma, Seno, } \\
\text { Tapoa, Yagha, } \\
\text { Comoe }\end{array}$ & supérieur & faible & $\begin{array}{l}\text { moyenne/ } \\
\text { élevée }\end{array}$ & faible & $\begin{array}{l}\text { Renforcement des capacités de I'agence de gestion } \\
\text { de l'eau de Gourma et Seno } \\
\text { Construction d'une unité d'engrais mineraux à Tapoa }\end{array}$ \\
\hline Kourweogo, Tuy & $\begin{array}{l}\text { inférieur/ } \\
\text { supérieur }\end{array}$ & $\begin{array}{l}\text { moyenne / } \\
\text { élevée }\end{array}$ & faible & $\begin{array}{l}\text { moyenne / } \\
\text { élevée }\end{array}$ & Non metionné dans le PNSR2 \\
\hline $\begin{array}{l}\text { Bougouriba, } \\
\text { Ziro }\end{array}$ & supérieur & medium & faible & faible & Construction d'un barrage à Bougouriba \\
\hline $\begin{array}{l}\text { Loroum, Ya- } \\
\text { tenga, Oudalan, } \\
\text { Soum, Ioba }\end{array}$ & $\begin{array}{l}\text { inférieur / } \\
\text { supérieur }\end{array}$ & $\begin{array}{l}\text { moyenne / } \\
\text { élevée }\end{array}$ & $\begin{array}{l}\text { moyenne / } \\
\text { élevée }\end{array}$ & faible & $\begin{array}{l}\text { Construction d'un barrage hydro-agricole et élec- } \\
\text { trique à Ouessa et d'un barrage à Bambakari/Tin- } \\
\text { Akoff }\end{array}$ \\
\hline Poni & supérieur & moyenne & moyenne & moyenne & Non metionné dans le PNSR2 \\
\hline
\end{tabular}

Notes : L'ensemble des inefficacités définissant chaque groupe de provinces hautement prioritaires est indiqué en gras.

Source : Les Auteurs, à partir des données Brown de Colstoun et al. (2017); CFSVA (2012); DHS (2010); Hansen et al. (2013); Ministère de I'Agriculture du Burkina Faso (2010, 2016); Pekel et al. (2016); SP/CPSA (2017); Stadlmayr et al. (2012); UNEP-WCMC (2018); Xiong et al. (2017). 


\section{NOTE DE PLAIDOYER DU CHAPITRE 2}

Les résultats de la typologie de ce chapitre peuvent être utiles doublement au travail de plaidoyer des OSC au Burkina Faso. Premièrement, ils facilitent le plaidoyer en faveur de politiques de SAN plus sensibles à la géographie et tenant compte des différences entre les provinces. Deuxièmement, les résultats nous aident à remettre en question ou à contester les investissements qui ne ciblent pas les obstacles les plus critiques à l'amelioration des résultats en matière de nutrition.

Deux questions de base doivent être soulevées lors de l'évaluation des politiques gouvernementales actuelles ou futures en matière de SAN :

1) Quel type d'obstacles cette politique vise-t-elle ? S'agit-il d'un problème ou d'une contrainte de production, d'accès ou d'utilisation?

2) Quelle zone, région ou province du pays cette politique vise-t-elle?

\section{ACTIONS POSSIBLES}

- Si les politiques ne sont pas ciblées sur le plan géographique, les OSC peuvent se référer au Tableau 2.2. qui énumère les provinces hautement prioritaires et leurs obstacles les plus critiques.

- Si la politique mentionne explicitement des zones cibles, alors :

- Les OSC pourraient veiller à ce que les priorités s'alignent sur celles qui sont résumées dans le Tableau 2.2.

- Lorsque les politiques ciblent les provinces à faible ou moyenne priorité, les OSC pourraient réorienter l'attention vers les zones à haute priorité. Les OSC pourraient également demander que ces politiques garantissent que les investissements dans les provinces à faible priorité bénéficient également aux provinces à haute priorité qui les entourent.

- Lorsque les politiques des provinces hautement prioritaires ne donnent pas la priorité aux obstacles les plus critiques, les OSC pourraient demander une réorientation afin que les interventions portent d'abord sur les problèmes les plus urgents. 


\section{Chapitre 3}

\section{CARTES D'ADÉQUATION DES NUTRIMENTS POUR DES INTERVENTIONS POLITIQUES CI- BLÉES}

\subsection{INTRODUCTION}

Ce chapitre présente une série de cartes d'adéquation des nutriments pour aider à comprendre, identifier et localiser les principaux défis qui se cachent derrière l'insuffisance ou le déséquilibre de l'apport alimentaire dans le pays. Les cartes montrent les données de production et de consommation alimentaire converties en quantités correspondantes de calories et de nutriments, en les comparant aux besoins nutritionnels de la population de chaque région. La méthode adoptée s'aligne globalement sur l'approche du système alimentaire actuellement préconisée par de nombreux chercheurs et partenaires de développement (Ericksen, 2008; Gillespie \& van den Bold, 2017; Global Panel on Agriculture and Food Systems for Nutrition, 2016; Jones \& Ejeta, 2015; PinstrupAndersen, 2013; Stephens, Jones, \& Parsons, 2018; Tendall et al., 2015). Dans ce chapitre, nous nous concentrons sur les calories totales consommées (apport énergétique) ainsi que sur un ensemble de micronutriments associés à la « faim cachée » lorsqu'ils sont consommés en quantités insuffisantes. Il s'agit notamment du calcium, du fer, du zinc, du folate, de la vitamine B12 et de la vitamine A. Même en l'absence de signes évidents de malnutrition comme le retard de croissance ou la faim « ressentie », les carences en micronutriments affectent le bien-être et le développement d'une personne et peuvent entraîner des troubles mentaux et cognitifs, une mauvaise santé, une faible productivité, voire la mort. En comparant l'adéquation de la consommation de nutriments avec les produits alimentaires disponibles localement qui contiennent des micronutriments essentiels, nous pouvons concevoir les politiques de SAN de manière stratégique.

La Figure 3.1. présente le défi global de la sous-nutrition au Burkina Faso, exprimé à la fois en termes de quantité et de qualité du régime alimentaire. Alors que la quantité du régime alimentaire se réfère à un apport suffisant en kilocalories, la qualité du régime alimentaire concerne l'apport en micronutriments. Dans ce chapitre, nous définissons l'adéquation des nutriments au «niveau du ménage », ce qui signifie que le nombre requis de kilocalories ou de micronutriments pour chaque ménage dépend de la taille et

\section{Mots clés}

\section{Adéquation des nutriments :}

Apport suffisant de nutriments essentiels en fonction des besoins individuels pour une santé optimale.

Micronutriments : Eléments essentiels requis en petites quantités ; les vitamines et les minéraux sont deux ensembles distincts de micronutriments.

\section{Contraintes de la demande : Des}

limites définies par le caractère abordable des produits pour le consommateur ou la volonté du consommateur de payer pour un certain produit.

\section{Equivalence adulte-homme :}

Concept pour standardiser les besoins à travers des groupes de population.

\section{Poids d'échantillonnage : Valeurs}

associées aux observations de données pour garantir que les statistiques des enquêtes sont représentatives de la population. de la structure démographique de la famille. Pour obtenir des taux moyens d'adéquation pour chaque région, nous plafonnons d'abord les taux d'adéquation énergétique et nutritionnelle des ménages à 100\% (étant entendu que $100 \%$ signifie un apport suffisant). La quantité du régime alimentaire est simple puisqu'elle ne dépend que du total de kilocalories ; pour la qualité du régime alimentaire, nous appliquons la même procédure pour chaque micronutriment individuellement avant de calculer la moyenne arithmétique au niveau du ménage, également connue sous le nom de ratio d'adéquation moyen (MAR).

La carte de gauche de la Figure 3.1. montre que l'apport énergétique alimentaire est légèrement meilleur dans trois régions du Nord-Est - le Sahel, le Centre-Nord et l'Est - qui ont des niveaux d'adéquation supérieurs à $90 \%$. En allant vers I'Ouest, les ménages vivant dans le Centre-Sud, le Centre-Est, le Sud-Ouest et surtout ceux de la région Centre, obtiennent des résultats relativement moins bons. En ce qui concerne l'adéquation des micronutriments (carte de droite de la Figure 3.1.), nous constatons une disparité similaire entre le Nord-Est et le reste du pays ; le Centre, le Centre-Est et les Haut-Bassins étant les plus touchés par la malnutrition de la « faim cachée ». La capitale Ouagadougou et la région Centre qui l'entoure présentent de graves carences, tant en quantité qu'en qualité, et leurs adéquations en kilocalories et en micronutriments sont inférieurs à $70 \%$ et $40 \%$, respectivement. 


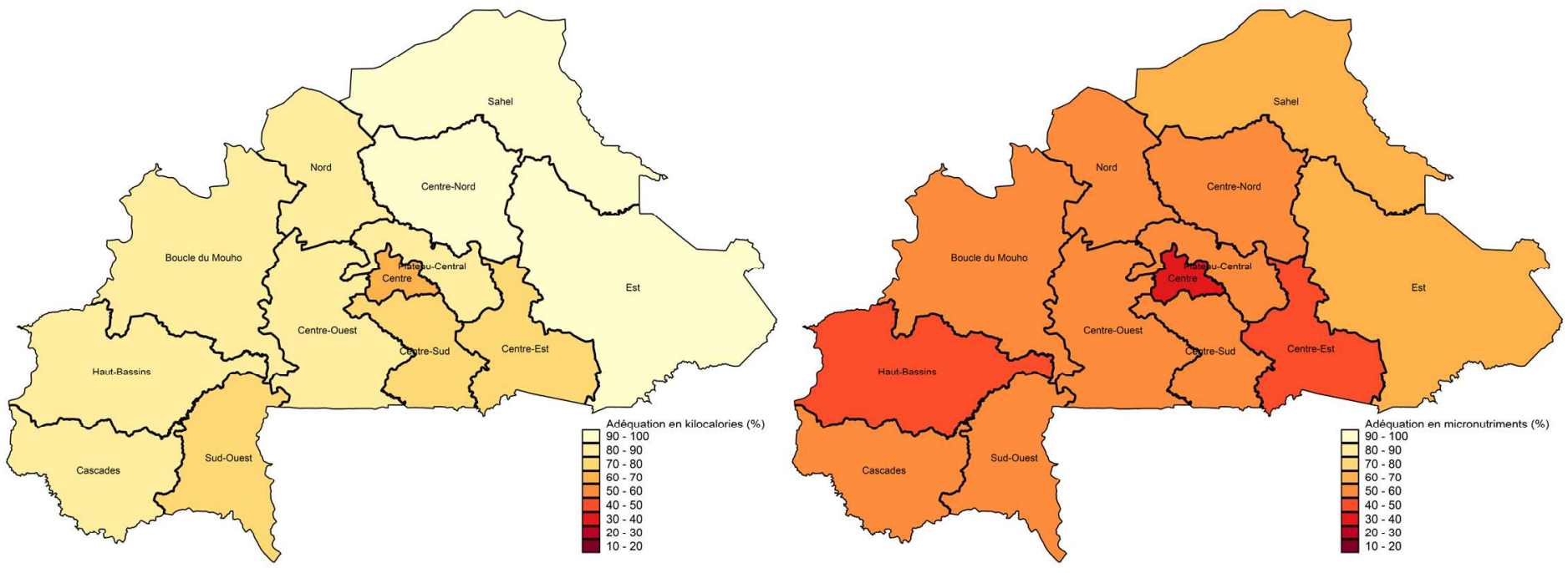

Source : Les Auteurs, à partir des données EMC (2014).

\subsection{CADRE CONCEPTUEL}

Afin de générer des données probantes sur les causes de l'hétérogénéité spatiale de la carence de l'apport alimentaire ainsi que les possibilités connexes d'y remédier, nous cartographions trois mesures distinctes d'adéquation des nutriments pour l'énergie alimentaire et les micronutriments. La première mesure, l'adéquation de la production de nutriments, quantifie la capacité de la production agricole de la région à satisfaire les besoins minimaux en énergie et en nutriments de sa population. La deuxième mesure, l'adéquation du marché des nutriments, indique l'accessibilité agrégée des calories et des nutriments, en fonction des besoins alimentaires de la population de la région. La troisième mesure, l'adéquation des nutriments au niveau des ménages, concerne l'inégalité d'accès parmi les ménages de chaque région. En comparant les trois cartes d'adéquation des nutriments et en les reliant à leurs sources alimentaires correspondantes, nous pouvons concevoir et évaluer stratégiquement les politiques de SAN.

La couleur verte de la Figure 3.2. indique un surplus de nutriments dans une zone, le rouge indique un déficit et le blanc indique I'autosuffisance. Le panneau (a) de la Figure 3.2. montre trois zones avec une production insuffisante de nutriments (zones B, C et $\mathrm{D}$ ). Augmenter la production de nutriments dans ces zones pourrait être une stratégie politique appropriée, mais il est important d'explorer d'abord les défis et les opportunités supplémentaires. Par exemple, regardez la zone D dans les panneaux (a) et (b). Même si la zone $D$ ne produit pas suffisamment de nutriments, les zones voisines $E$ et $F$ présentent des excédents de production - le marché de la zone $D$ n'est pas déficitaire en nutriments, peut-être grâce à une intégration réussie du marché avec les zones $E$ et $F$. Compte tenu de sa situation géographique, la zone $C$ pourrait rechercher des formes similaires d'intégration du marché avec les zones $\mathrm{E}$ et $\mathrm{F}$. 


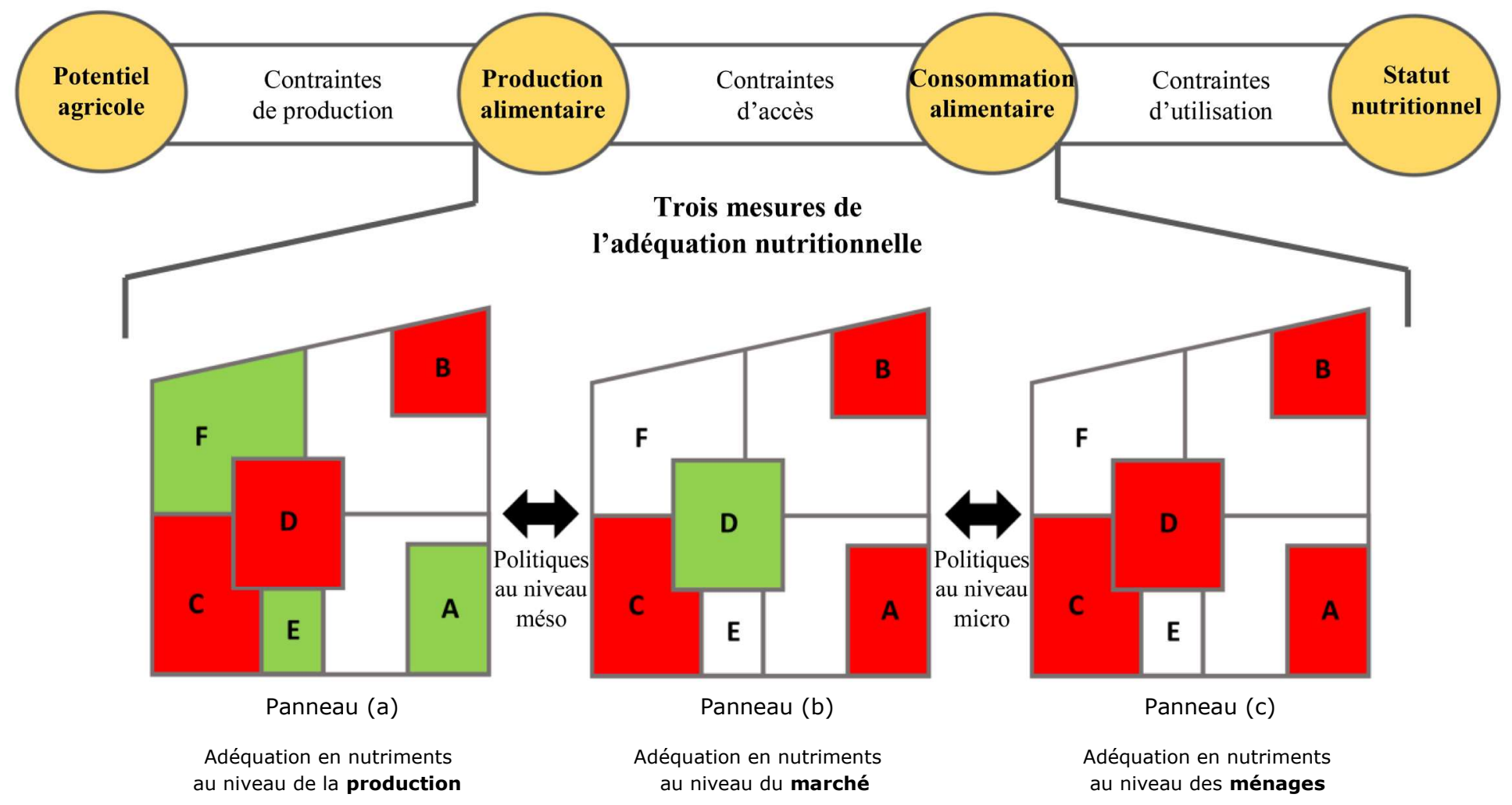

Note : Le blanc indique l'autosuffisance, le vert l'excédent et le rouge l'insuffisance.

Source : Les Auteurs.

Examinez maintenant la zone A dans les panneaux (a) et (b). La zone A produit suffisamment de nutriments, mais les marchés de la zone $A$ ne vendent pas assez pour maintenir la population locale en bonne santé. C'est ce que nous appelons la « perte de nutriments ». Elle se produit lorsque les nutriments produits localement sont commercialisés ou vendus en dehors de la zone, ou lorsque les pertes post-récolte empêchent ces aliments riches en nutriments d'arriver sur les marchés locaux. Pour remédier à la perte de nutriments, il peut être nécessaire de revoir les politiques commerciales ou d'échanges, d'améliorer les infrastructures de transport afin de mieux relier les marchés aux producteurs, ou d'investir dans des capacités de stockage et de transformation.

Enfin, comparons la zone D dans les panneaux (b) et (c). Bien que suffisamment de nutriments parviennent au marché (panneau b), les ménages ne disposent toujours pas d'une alimentation nutritive adéquate (panneau c). Pourquoi en serait-il ainsi ? Peutêtre que les aliments nutritifs sont tout simplement trop chers pour les ménages; ou peut-être que les populations manquent de connaissances en matière de nutrition et ignorent donc les aliments riches en nutriments. Nous appelons ces obstacles des contraintes de la demande car elles ne dépendent pas des fournisseurs mais des consommateurs qui demandent des produits alimentaires. Pour relever ces défis, il peut être nécessaire de mettre en place des mesures de protection sociale tenant compte de la nutrition et contribuant à compléter les revenus, ou de mener des campagnes de sensibilisation qui font la promotion des aliments riches en nutriments ou d'inclure une éducation nutritionnelle.

\subsection{LES DONNEES}

Les données utilisées dans ce chapitre proviennent de différentes sources mais chaque source nous permet de calculer des estimations de l'adéquation des nutriments pour les 13 régions du Burkina Faso. Pour l'adéquation de la production, nous combinons les statistiques officielles de production de 2014 pour cinq céréales principales (maïs, riz, mil, sorgho et fonio), deux tubercules (patate douce et igname), quatre légumineuses (niébé, arachide, sésame et voandzou) et divers produits d'élevage comprenant différents types de viande, de lait et d'œufs (Ministère de l'Agriculture du Burkina Faso, 2015; Ministère des Ressources Animales du Burkina Faso, 2015). En outre, nous estimons la production de fruits, de légumes et de poissons en actualisant les chiffres de 2008 à l'aide d'un ratio constant productivité/population. Ces données couvrent 16 produits horticoles et sept espèces de poissons (Ministère de l'Agriculture et de la Sécurite Alimentaire du Burkina Faso, 2014). En utilisant les données sur la composition des aliments compilées pour l'Afrique de l'Ouest (Stadlmayr et al., 2012), nous convertissons les quantités de production en kilocalories et autres équivalents nutritifs et les agrégeons par région. Nous estimons le ratio d'adéquation de la production en divisant les niveaux de production d'énergie alimentaire et de nutriments par leurs apports respectifs requis en fonction de la composition 
démographique de chaque région. Nous utilisons la même approche pour l'adéquation du marché, en nous basant sur les données de consommation alimentaire obtenues avec I'Enquête Multisectorielle Continue (EMC) menée au Burkina Faso en 2014 (Institut National de la Statistique et de la Démographie du Burkina Faso, 2016). Cette enquête combine différentes sources de consommation alimentaire, telles que les achats, les denrées produites à la maison, les dons en nature et l'épuisement des stocks de nourriture, enregistrés lors de quatre visites au cours de l'année. Au total, plus de 50 produits alimentaires sont couverts. Les quantités de produits alimentaires achetés sont estimées en imputant les prix alimentaires régionaux. En utilisant les mêmes données sur la composition des aliments (Stadlmayr et al., 2012), nous dérivons la consommation agrégée de nutriments par région et la divisons par les niveaux d'apport requis pour la région.

Nous dérivons l'adéquation au niveau des ménages de la même enquête sur la consommation des ménages, estimée au niveau des ménages en considérant l'apport nécessaire correspondant à la famille (en plafonnant toutes les valeurs supérieures à $100 \%$ ) puis en faisant la moyenne des ratios des ménages par région. Les niveaux d'apport requis pour chaque ménage et chaque région sont déterminés en utilisant l'approche d'équivalence adulte-homme combinée à des poids d'échantillonnage de la population. Chacune des trois mesures est un ratio, où $100 \%$ reflète l'adéquation, et les valeurs inférieures (supérieures) à $100 \%$ indiquent une carence (excédent). La couleur utilisée dans ce chapitre est le jaune pâle pour l'adéquation, le vert pour l'excédent et le rouge pour la carence.

\subsection{ADEQUATION DES NUTRIMENTS}

Le Tableau 3.1. donne un aperçu des écarts absolus en matière de production et de consommation de nutriments, par rapport aux niveaux d'apport recommandés ainsi que les trois mesures d'adéquation des nutriments. Le Burkina Faso présente de bons résultats en matière de consommation de kilocalories et de protéines : les ménages consomment en moyenne au moins $80 \%$ de I'apport recommandé. Malheureusement, les carences en micronutriments sont alarmantes, en particulier pour la vitamine B12 $(28 \%)$, le calcium (33\%), la vitamine A (35\%) et le fer $(63 \%)$. La faible adéquation de la production - seulement $6 \%$ pour la vitamine B12 et 35\% pour la vitamine A - semble être à I'origine de ces carences. Le Burkina Faso semble importer de nombreux aliments riches en vitamine B12, ce qui compense quelque peu la pénurie de production, mais les importations ne touchent peutêtre qu'une population limitée.

Le Burkina Faso ne produit pas non plus suffisamment d'aliments riches en calcium, mais la principale préoccupation concerne les « pertes » survenant entre la production et les marchés. Le pays produit $85 \%$ de l'apport requis en calcium, mais l'adéquation au niveau du marché n'est que de 33\%, ce qui indique soit des pertes alimentaires soit des exportations de produits alimentaires riches en calcium. Les pertes de nutriments affectent tous les autres nutriments, mais avec des conséquences moindres sur la santé grâce aux excédents de production de fer, de zinc, de folate, ainsi que de kilocalories et de protéines. Pour le zinc, le folate et les protéines, l'inégalité d'accès aux nutriments disponibles localement exacerbe les carences en nutriments, comme on peut le déduire des baisses importantes entre l'adéquation du marché et l'adéquation au niveau des ménages.

Table 3.1. Niveaux nationaux d'énergie alimentaire et d'adéquation en nutriments basés sur la production, la consommation et les apports recommandés, Burkina Faso (2014)

\begin{tabular}{|c|c|c|c|c|c|c|}
\hline & $\begin{array}{l}\text { Production } \\
\text { Par jour, AME }\end{array}$ & $\begin{array}{l}\text { Consommation } \\
\text { Par jour, AME }\end{array}$ & $\begin{array}{c}\text { Apport recom- } \\
\text { mandé } \\
\text { Par jour, AME }\end{array}$ & $\begin{array}{c}\text { Adéquation au } \\
\text { niveau de la } \\
\text { production } \\
(\%)\end{array}$ & $\begin{array}{c}\text { Adéquation au } \\
\text { niveau du } \\
\text { marché } \\
(\%)\end{array}$ & $\begin{array}{c}\text { Adéquation au } \\
\text { niveau des } \\
\text { ménages } \\
(\%)\end{array}$ \\
\hline $\begin{array}{l}\text { Kilocalories } \\
\text { (kcal) }\end{array}$ & 4690,1 & 2523,1 & 2750,0 & 170,5 & 91,7 & 81,4 \\
\hline $\begin{array}{l}\text { Protéines } \\
\text { (g.) }\end{array}$ & 158,9 & 76,7 & 50,0 & 317,7 & 153,4 & 95,7 \\
\hline $\begin{array}{l}\text { Calcium } \\
\text { (mg.) }\end{array}$ & 848,0 & 329,4 & 1000,0 & 84,8 & 32,9 & 33,3 \\
\hline $\begin{array}{l}\text { Iron } \\
\text { (mg.) }\end{array}$ & 39,4 & 18,2 & 27,4 & 144,0 & 66,4 & 62,5 \\
\hline $\begin{array}{l}\text { Zinc } \\
(\mathrm{mg} .)\end{array}$ & 28,7 & 12,4 & 14,0 & 205,3 & 88,9 & 77,3 \\
\hline $\begin{array}{l}\text { Folate } \\
\text { (mcg.) }\end{array}$ & 844,9 & 378,4 & 400,0 & 211,2 & 94,6 & 79,1 \\
\hline $\begin{array}{l}\text { Vitamine B12 } \\
\text { (mcg.) }\end{array}$ & 0,1 & 0,7 & 2,4 & 5,9 & 28,8 & 27,9 \\
\hline $\begin{array}{l}\text { Vitamine A } \\
\text { (mcg.) }\end{array}$ & 209,7 & 213,9 & 600,0 & 34,9 & 35,6 & 35,4 \\
\hline
\end{tabular}

Source : Les Auteurs, avec les données EMC (2014); Ministère de l'Agriculture (2015); Ministère de l'Agriculture et de la Securite Alimentaire (2014); Ministère des Ressources Animales (2015).

La Figure 3.3. présente les trois mesures d'adéquation pour chacun des six micronutriments couverts dans ce chapitre, illustrés géographiquement par région. 
Notez dans le panneau (e) que la production insuffisante de vitamine $B 12$ affecte les 13 régions du pays : elles présentent toutes une adéquation de la production bien inférieure à 30\% (carte de gauche). En raison des importations de produits alimentaires riches en vitamine B12, la situation est moins dramatique au niveau du marché (carte du milieu), en particulier pour les régions occidentales du pays et le Centre-Sud. Ce schéma spatial est similaire au niveau des ménages (carte de droite), Cascades et le Sud-Ouest présentant des ratios d'adéquation des nutriments légèrement supérieurs à $40 \%$, alors que les ménages de la plupart des régions consomment (en moyenne) moins de $30 \%$ de ce qui est recommandé. Pour résoudre le problème de la grave carence en vitamine B12, le Burkina Faso pourrait mettre en œuvre des programmes de protection sensibles à la nutrition, tels que la supplémentation en vitamine B12 grâce à des aliments fortifiés, l'augmentation de la production de produits d'origine animale ou la mise en place de politiques commerciales qui encouragent les importations de produits alimentaires d'origine animale.

Les carences de la production de vitamine $A$ sont plus hétérogènes à travers le pays (voir le panneau f). C'est dans le Sud-Ouest que l'on observe les plus fortes adéquations de production, les Haut-Bassins étant la seule région à produire un excédent. Dans le Nord-Est, les adéquations en vitamine A sont inférieures à 30\%. Ce schéma spatial n'est pas surprenant étant donné les pluies abondantes dans le Sud-Ouest, où poussent des fruits tropicaux comme la mangue et la papaye (toutes deux riches en vitamine A et selon les statistiques officielles, principalement produites dans les Haut-Bassins). Si l'on compare la production de vitamine A et l'adéquation des marchés, on constate des pertes de nutriments dans le Sud-Ouest, qui pourraient être interprétées comme des pertes de produits alimentaires entre les sites de production et les marchés ou comme des exportations vers les régions voisines. Comme l'adéquation en vitamine A dans I'Est (en particulier dans la région Est) augmente effectivement entre l'etape de la production et celle du marché, il semble que cette région bénéficie d'aliments importés riches en vitamine $A$. L'adéquation au niveau des ménages est plus élevée à Cascades et à l'Est avec des ratios supérieurs à 50\%, et plus faible dans la région Nord. En termes de recommandations politiques, la production d'aliments riches en vitamine A doit augmenter dans les zones agricoles appropriées, les pertes doivent diminuer et les aliments doivent être distribués dans les régions où les conditions agroécologiques sont moins favorables.

La carence en calcium (voir panneau a) constitue une menace majeure, l'adéquation au niveau des ménages n'approchant pas les $50 \%$ et étant d'une faiblesse alarmante dans le Nord et le Centre (carte de droite). La carte de l'adéquation au niveau des ménages imite la faible adéquation du marché (carte du milieu). Pourtant, quatre régions (Boucle du Mouhoun, Est, Cascades et CentreOuest) produisent suffisamment de calcium pour nourrir leurs populations respectives. Cela indique des niveaux importants de perte de nutriments, soit à travers des pertes alimentaires, soit à travers le commerce. La culture du sésame pour les marchés d'exportation internationaux explique probablement cette perte. Après le coton, le sésame est la plus importante culture d'exportation du Burkina Faso (Glin, Mol, \& Oosterveer, 2013), et il est extrêmement riche en calcium (soit 983 mg par $100 \mathrm{~g}$ de portion comestible). Dans toutes les autres régions, notamment dans le Nord, la production de calcium est inférieure aux besoins (suivie par l'inadéquation des marchés et au niveau des ménages). En plus de stimuler la production d'autres produits alimentaires riches en calcium, les agriculteurs du Burkina Faso pourraient être encouragés à produire davantage de sésame pour leur propre marché intérieur.

Pour le fer (panneau b), le zinc (panneau c) et le folate (panneau d), la configuration spatiale des obstacles est en grande partie la même : on observe une perte substantielle de nutriments combinée à des contraintes de demande. La production de ces trois micronutriments semble suffisante, avec des adéquations supérieures à $100 \%$ dans toutes les régions (sauf au Centre). Cependant, en comparant l'adéquation de la production et l'adéquation du marché, nous constatons d'énormes réductions de la disponibilité des nutriments dans tout le pays (moins au Sahel, dans le Centre-Nord et dans l'Est). De même, les adéquations en nutriments des ménages sont généralement inférieures à celles du marché. La baisse est moins grave pour le fer, mais elle suggère néanmoins que les ménages rencontrent des obstacles (économiques, comportementaux, éducatifs) à l'achat d'aliments riches en fer, en zinc et en folate.

Pour terminer cette section, nous attirons l'attention sur la situation d'insécurité alimentaire grave de Ouagadougou, située dans la région Centre. En plus d'une très faible adéquation de la production de nutriments due à la proportion limitée de terres arables et à la forte population, la capitale semble mal reliée à ses propres marchés alimentaires nationaux (ou internationaux). Pour tous les nutriments couverts dans ce chapitre, la région Centre figure toujours parmi les régions où les marchés des nutriments sont les moins adéquats, ce qui compromet sérieusement l'accès des ménages à une alimentation saine et diversifiée. 

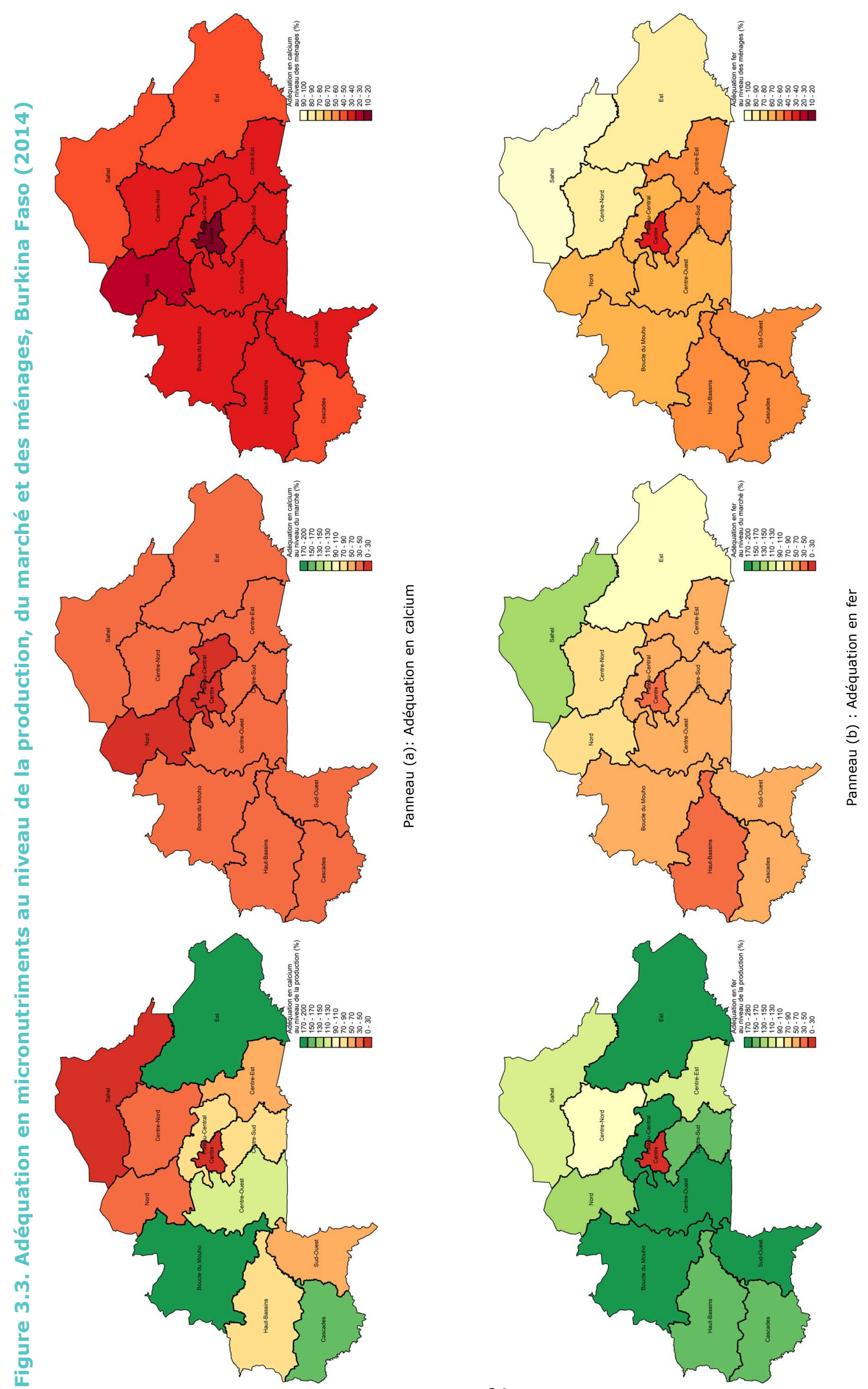

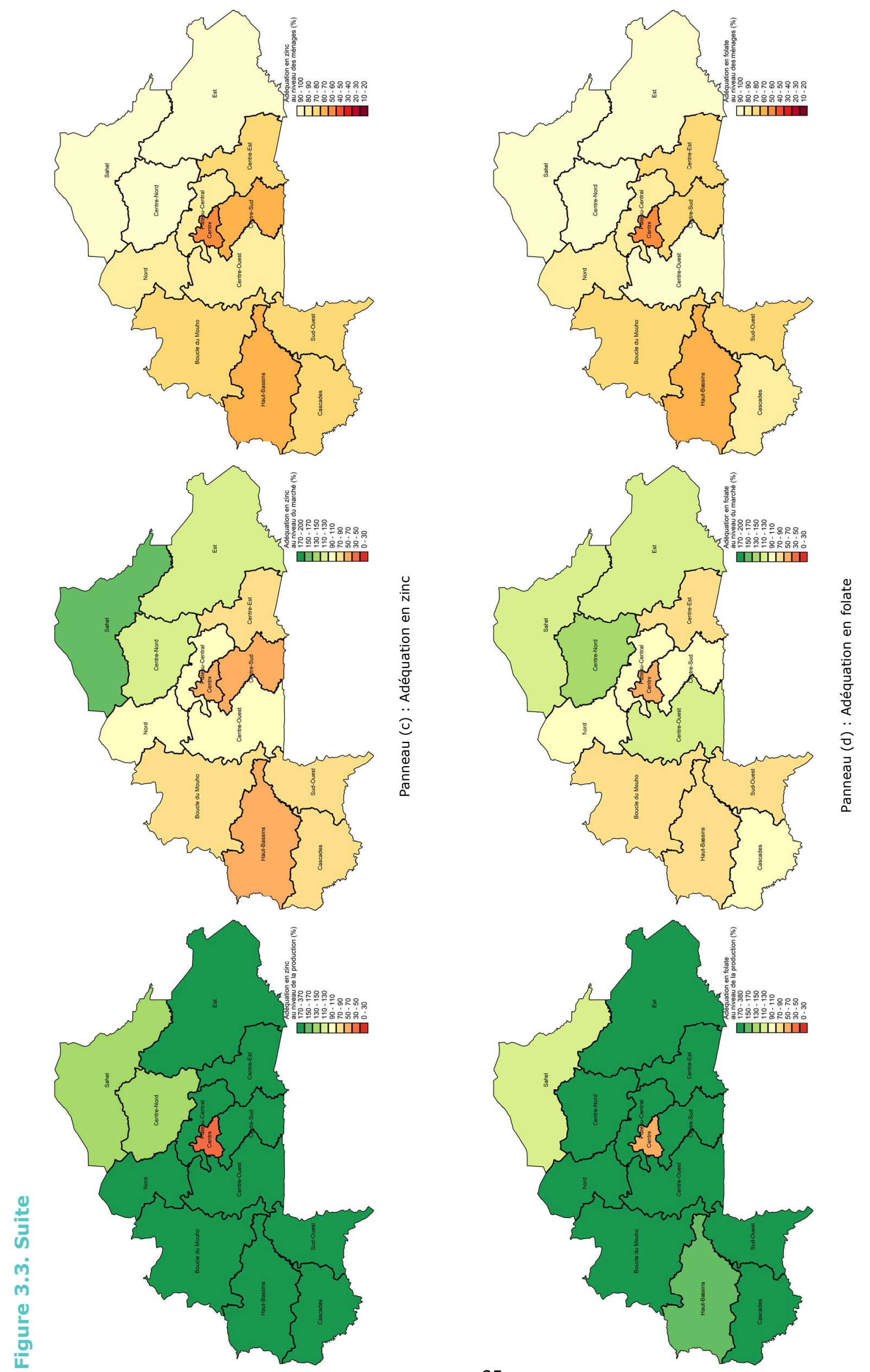

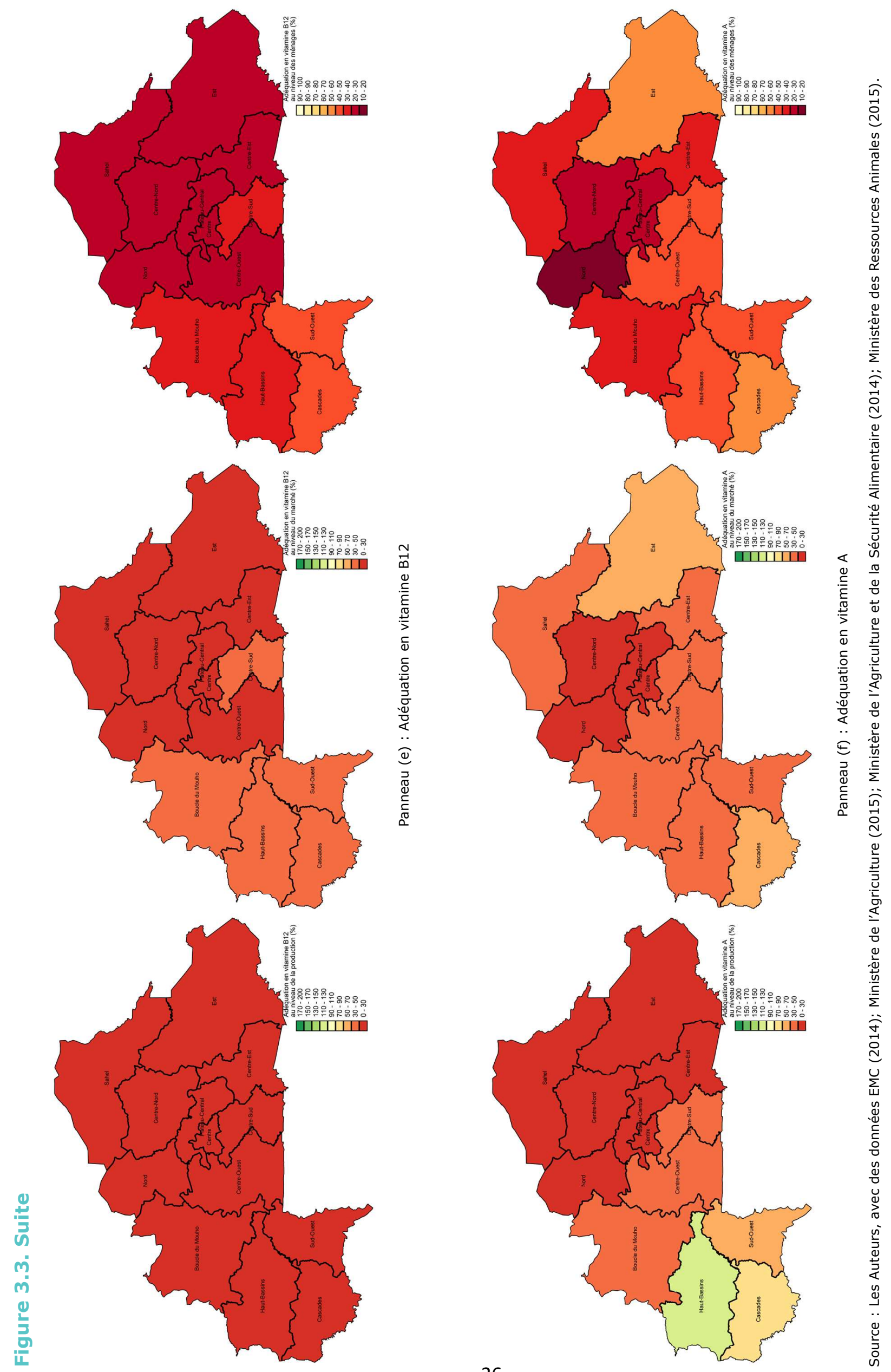


\subsection{RETOUR AUX PRODUITS ALIMENTAIRES ET POLITIQUES CIBLEES A PARTIR DES CARENCES NUTRITIONNELLES}

Dans cette section, nous nous concentrons sur les produits alimentaires réels qui fournissent des micronutriments, en considérant les recommandations politiques qui pourraient aider à augmenter la production, à réduire la perte de nutriments et à améliorer I'accès des consommateurs à des aliments sains. Dans la vie réelle, les populations cultivent et achètent des aliments réels - et non des micronutriments. Pour chaque nutriment couvert dans ce chapitre, le Tableau 3.2. présente les cinq denrées alimentaires nationales les plus importantes selon deux critères :

1) part actuelle dans l'apport global de nutriments (\%) (colonne de gauche)

2) les prix les moins chers par nutriment (exprimés en francs CFA) (colonne de droite)

Le Tableau 3.2. indique également le contenu nutritionnel par portion comestible de 100 grammes de chaque produit alimentaire (Stadlmayr et al., 2012) et sa part dans le budget alimentaire.

L'augmentation de la production ou de l'importation d'aliments d'origine animale pourrait contribuer à remédier à la carence en vitamine B12 au Burkina Faso. Une plus grande disponibilité d'aliments d'origine animale contribuera à faire baisser les prix et à augmenter la consommation des ménages. Actuellement, la viande est au moins cinq fois plus chère que le poisson séché, ce qui signifie que l'augmentation de la production animale pourrait avoir un impact plus dynamique. La fortification des aliments en vitamine B12 pourrait également améliorer l'apport en vitamine B12. À court terme, les ménages pourraient consommer des suppléments en vitamine B12, ou réaffecter une partie de leur budget alimentaire de manière à dépenser davantage en poisson (séché).

De même, la stimulation de la production d'aliments riches en vitamine A dans les zones où les conditions biophysiques sont favorables pourrait augmenter l'apport. Les légumes à feuilles (comme les feuilles d'oseille et de baobab) sont très riches en vitamine $A$; la patate douce est légèrement plus rentable (mais moins consommée à travers le pays), avec un prix des nutriments de 0,09 CFA par mcg de vitamine A (contre 0,11 CFA par mcg pour les légumes à feuilles). Étant donné que ces produits alimentaires sont comparables en termes de prix, et qu'ils figurent tous deux dans les cinq premiers sur les listes du Tableau 3.2., nous suggérons que le plus grand impact nutritionnel viendra de l'augmentation de la production plutôt que de la modification des préférences alimentaires.

De même, la production d'aliments riches en calcium devrait augmenter l'apport en calcium. Comme le montre le Tableau 3.2., le poisson séché pourrait à nouveau être un produit potentiel viable, étant donné son prix des nutriments relativement bas et sa forte densité en calcium (c'est-à-dire $904 \mathrm{mg}$ par $100 \mathrm{~g}$ de portion comestible). Cette stratégie pourrait être combinée avec des politiques visant à promouvoir la production de sésame pour la consommation intérieure. Actuellement, le sésame est pratiquement absent du panier alimentaire quotidien car la majeure partie de la production est exportée vers les marchés internationaux. Ajoutant ainsi à la plus grande disponibilité de matières premières sur le marché intérieur, les graines de sésame pourraient être transformées en produits tels que les barres de sésame afin de répondre plus facilement aux besoins en calcium dans les régions éloignées.

Les cinq sources alimentaires les plus importantes (colonne de gauche) pour le fer, le zinc et le folate, ainsi que pour les kilocalories et les protéines, sont remarquablement similaires aux alternatives alimentaires rentables (colonne de droite). Pour les trois micronutriments, chaque liste des cinq principaux produits contient les mêmes céréales et légumineuses : le mil, le sorgho, le maïs et les haricots. En l'absence d'alternatives alimentaires rentables pour augmenter l'apport en fer, en zinc et en folate, ce régime alimentaire peu diversifié est également un signe de la pauvreté et du caractère peu abordable des produits. L'augmentation de la disponibilité des céréales et des légumineuses sur le marché devrait rendre ces produits plus abordables pour la population burkinabé. Comme le pays semble être autosuffisant pour la production de chacun de ces micronutriments, la réduction des pertes entre la production de céréales et de légumineuses et leur distribution sur le marché pourrait contribuer à remédier aux carences en fer, en zinc et en folate. L'exportation de sésame riche en fer et en zinc offre également une explication partielle. Le Burkina Faso devra avant tout déterminer la raison des pertes alimentaires, d'autant plus que le pays est un importateur net de céréales, qui sont des denrées alimentaires sèches (et donc vraissemblablement résistantes à la détérioration).

Tout en s'efforçant d'augmenter la production agricole de certaines cultures et de réduire les pertes en nutriments pour d'autres, les régimes de protection sociale pourraient contribuer à assurer des apports en nutriments essentiels aux familles les plus sousalimentées du Burkina Faso. 
Tableau 3.2. Les 5 principaux produits alimentaires en fonction de la part et du prix des nutriments, Burkina Faso (2014)

\begin{tabular}{|c|c|c|c|c|c|c|c|c|c|c|}
\hline & Produit alimentaire & $\begin{array}{l}\text { Part des } \\
\text { apports } \\
\text { en nutri- } \\
\text { ments }\end{array}$ & $\begin{array}{c}\text { Prix par } \\
\text { nutri- } \\
\text { ment } \\
\text { (FCFA) }\end{array}$ & $\begin{array}{c}\text { Teneur } \\
\text { en nutri- } \\
\text { ments } \\
100 \mathrm{gr}\end{array}$ & $\begin{array}{l}\text { Part du } \\
\text { budget } \\
\text { alimen- } \\
\text { taire }\end{array}$ & Produit alimentaire & $\begin{array}{c}\text { Part des } \\
\text { apports } \\
\text { en nutri- } \\
\text { ments }\end{array}$ & $\begin{array}{c}\text { Prix par } \\
\text { nutri- } \\
\text { ment } \\
\text { (FCFA) }\end{array}$ & $\begin{array}{c}\text { Teneur } \\
\text { en nutri- } \\
\text { ments } \\
\text { 100gr }\end{array}$ & $\begin{array}{l}\text { Part du } \\
\text { budget } \\
\text { alimen- } \\
\text { taire }\end{array}$ \\
\hline \multirow{5}{*}{$\begin{array}{l}y \\
\frac{0}{2} \\
\frac{0}{\pi}\end{array}$} & Sorgho & $29,0 \%$ & 0,05 & 352,5 & $14,0 \%$ & Maïs & $25,3 \%$ & 0,04 & 351,0 & $10,7 \%$ \\
\hline & Maïs & $25,3 \%$ & 0,04 & 351,0 & $10,7 \%$ & Sorgho & $29,0 \%$ & 0,05 & 352,5 & $14,0 \%$ \\
\hline & Mil & $15,5 \%$ & 0,05 & 382,0 & $8,2 \%$ & Mil & $15,5 \%$ & 0,05 & 382,0 & $8,2 \%$ \\
\hline & Riz & $10,9 \%$ & 0,11 & 349,0 & $12,6 \%$ & Karité & $1,2 \%$ & 0,08 & 900,0 & $1,0 \%$ \\
\hline & Haricots & $5,1 \%$ & 0,08 & 335,0 & $4,5 \%$ & Haricots & $5,1 \%$ & 0,08 & 335,0 & $4,5 \%$ \\
\hline \multirow{5}{*}{ 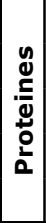 } & Sorgho & $27,9 \%$ & 1,70 & 9,6 & $14,0 \%$ & Haricots & $12,0 \%$ & 1,27 & 22,1 & $4,5 \%$ \\
\hline & Maïs & $23,1 \%$ & 1,57 & 9,1 & $10,7 \%$ & Maïs & $23,1 \%$ & 1,57 & 9,1 & $10,7 \%$ \\
\hline & Mil & $15,9 \%$ & 1,76 & 11,1 & $8,2 \%$ & Sorgho & $27,9 \%$ & 1,70 & 9,6 & $14,0 \%$ \\
\hline & Haricots & $12,0 \%$ & 1,27 & 22,1 & $4,5 \%$ & Mil & $15,9 \%$ & 1,76 & 11,1 & $8,2 \%$ \\
\hline & Riz & $7,5 \%$ & 5,70 & 6,9 & $12,6 \%$ & Arachides & $1,4 \%$ & 2,20 & 18,8 & $0,9 \%$ \\
\hline \multirow{5}{*}{$\frac{\xi}{\frac{5}{3}}$} & Legumes feuilles & $32,4 \%$ & 0,09 & 262,8 & $3,6 \%$ & Légumes feuilles & $32,4 \%$ & 0,09 & 262,8 & $3,6 \%$ \\
\hline & Sorgho & $16,4 \%$ & 0,68 & 24,0 & $14,0 \%$ & Poisson séché & $13,5 \%$ & 0,21 & 903,9 & $3,6 \%$ \\
\hline & Poisson séché & $13,5 \%$ & 0,21 & 903,9 & $3,6 \%$ & Patate douce & $0,7 \%$ & 0,34 & 35,6 & $0,3 \%$ \\
\hline & Haricots & $9,4 \%$ & 0,38 & 74,0 & $4,5 \%$ & Haricots & $9,4 \%$ & 0,38 & 74,0 & $4,5 \%$ \\
\hline & Maïs & $9,3 \%$ & 0,92 & 15,6 & $10,7 \%$ & Arachides & $1,2 \%$ & 0,62 & 67,0 & $0,9 \%$ \\
\hline \multirow{5}{*}{$\begin{array}{l}\frac{1}{凶} \\
\stackrel{L}{L}\end{array}$} & Mil & $28,6 \%$ & 2,51 & 7,8 & $8,2 \%$ & Mil & $28,6 \%$ & 2,51 & 7,8 & $8,2 \%$ \\
\hline & Sorgho & $28,2 \%$ & 4,32 & 3,8 & $14,0 \%$ & Sorgho & $28,2 \%$ & 4,32 & 3,8 & $14,0 \%$ \\
\hline & Maïs & $21,5 \%$ & 4,33 & 3,3 & $10,7 \%$ & Maïs & $21,5 \%$ & 4,33 & 3,3 & $10,7 \%$ \\
\hline & Haricots & $7,9 \%$ & 4,93 & 5,7 & $4,5 \%$ & Haricots & $7,9 \%$ & 4,93 & 5,7 & $4,5 \%$ \\
\hline & Légumes feuilles & $5,4 \%$ & 5,83 & 4,0 & $3,6 \%$ & Légumes feuilles & $5,4 \%$ & 5,83 & 4,0 & $3,6 \%$ \\
\hline \multirow{5}{*}{ 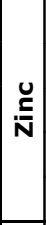 } & Sorgho & $30,6 \%$ & 7,96 & 2,1 & $14,0 \%$ & Mil & $21,4 \%$ & 6,70 & 2,9 & $8,2 \%$ \\
\hline & Mil & $21,4 \%$ & 6,70 & 2,9 & $8,2 \%$ & Haricots & $10,5 \%$ & 7,47 & 3,8 & $4,5 \%$ \\
\hline & Maïs & $21,1 \%$ & 8,81 & 1,6 & $10,7 \%$ & Sorgho & $30,6 \%$ & 7,96 & 2,1 & $14,0 \%$ \\
\hline & Haricots & $10,5 \%$ & 7,47 & 3,8 & $4,5 \%$ & Maïs & $21,1 \%$ & 8,81 & 1,6 & $10,7 \%$ \\
\hline & Riz & $6,5 \%$ & 33,66 & 1,2 & $12,6 \%$ & Arachides & $0,9 \%$ & 17,24 & 2,4 & $0,9 \%$ \\
\hline \multirow{5}{*}{$\begin{array}{l}0 \\
\frac{\pi}{0} \\
0\end{array}$} & Haricots & $40,4 \%$ & 0,07 & 395,0 & $4,5 \%$ & Haricots & $40,4 \%$ & 0,07 & 395,0 & $4,5 \%$ \\
\hline & Sorgho & $16,3 \%$ & 0,55 & 29,7 & $14,0 \%$ & Légumes feuilles & $11,6 \%$ & 0,20 & 117,5 & $3,6 \%$ \\
\hline & Maïs & $12,5 \%$ & 0,55 & 26,0 & $10,7 \%$ & Patate douce & $0,8 \%$ & 0,23 & 52,0 & $0,3 \%$ \\
\hline & Légumes feuilles & $11,6 \%$ & 0,20 & 117,5 & $3,6 \%$ & Arachides & $1,3 \%$ & 0,45 & 92,0 & $0,9 \%$ \\
\hline & Mil & $8,2 \%$ & 0,65 & 30,3 & $8,2 \%$ & Sorgho & $16,3 \%$ & 0,55 & 29,7 & $14,0 \%$ \\
\hline \multirow{5}{*}{ 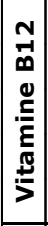 } & Poisson seche & $60,3 \%$ & 26,66 & 7,1 & $3,6 \%$ & Poisson séché & $60,3 \%$ & 26,66 & 7,1 & $3,6 \%$ \\
\hline & Viande de mouton/chèvre & $14,3 \%$ & 119,99 & 2,0 & $3,8 \%$ & Viande de mouton/chèvre & $14,3 \%$ & 119,99 & 2,0 & $3,8 \%$ \\
\hline & Lait & $8,1 \%$ & 218,83 & 0,6 & $3,9 \%$ & Poisson frais & $8,0 \%$ & 128,89 & 1,9 & $2,3 \%$ \\
\hline & Poisson frais & $8,0 \%$ & 128,89 & 1,9 & $2,3 \%$ & Poisson fume & $2,0 \%$ & 153,90 & 1,8 & $0,7 \%$ \\
\hline & Viande de bœuf & $6,7 \%$ & 177,99 & 1,1 & $2,6 \%$ & Viande de bœuf & $6,7 \%$ & 177,99 & 1,1 & $2,6 \%$ \\
\hline \multirow{5}{*}{ 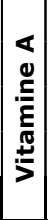 } & Légumes feuilles & $51,3 \%$ & 0,11 & 206,2 & $3,6 \%$ & Patate douce & $5,5 \%$ & 0,09 & 135,2 & $0,3 \%$ \\
\hline & Maïs & $30,2 \%$ & 0,57 & 25,0 & $10,7 \%$ & Légumes feuilles & $51,3 \%$ & 0,11 & 206,2 & $3,6 \%$ \\
\hline & Patate douce & $5,5 \%$ & 0,09 & 135,2 & $0,3 \%$ & Tomates fraiches & $4,5 \%$ & 0,55 & 52,0 & $1,5 \%$ \\
\hline & Tomates fraiches & $4,5 \%$ & 0,55 & 52,0 & $1,5 \%$ & Maïs & $30,2 \%$ & 0,57 & 25,0 & $10,7 \%$ \\
\hline & Haricots & $3,8 \%$ & 1,89 & 14,9 & $4,5 \%$ & Haricots & $3,8 \%$ & 1,89 & 14,9 & $4,5 \%$ \\
\hline
\end{tabular}

Note Note : Les colonnes grisées représentent les deux variables de classement utilisées pour établir les cinq principaux produits alimentaires pour chaque nutriment.

Source : Les Auteurs, avec les données EMC (2014). 


\section{NOTE DE PLAIDOYER DU CHAPITRE 3}

Examinons quelques messages clés de cette analyse :

1. La population du Burkina Faso souffre d'une carence en micronutriments, également connue sous le nom de «faim cachée », qui peut entraîner de graves problèmes de santé cognitive et physique et de développement humain.

2. Les causes de la faim cachée au Burkina Faso dépendent à la fois de la situation géographique, du type de micronutriment et des sources alimentaires correspondantes qui fournissent ce mi-

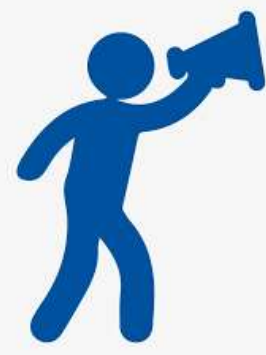
cronutriment.

3. Pour lutter contre la faim cachée, les interventions de politiques devraient être sensibles à la géographie et adaptées à des aliments spécifiques.

4. Le tableau ci-dessous résume les principaux résultats par micronutriment, en commençant par la vitamine B12, dont I'adéquation est la plus faible au niveau national. Les actions prioritaires proposées sont en gras et le texte souligné indique grossièrement les sites prioritaires.

\begin{tabular}{|c|c|c|}
\hline Nutriment & $\begin{array}{l}\text { Adéquation } \\
\text { des ménages } \\
\text { au niveau } \\
\text { national }\end{array}$ & Interventions de politiques ciblées \\
\hline $\begin{array}{l}\text { Vitamine } \\
\text { B12 }\end{array}$ & $27,9 \%$ & $\begin{array}{l}\text { - A long terme, augmenter la production (ou les importations) de produits alimen- } \\
\text { taires d'origine animale dans tout le pays. } \\
\text { À court terme, enrichir les aliments en vitamine B12, fournir des suppléments de } \\
\text { vitamine B12 ou mettre en œuvre des programmes de politiques visant à réaffecter les } \\
\text { budgets alimentaires des ménages pour augmenter les dépenses en poisson (séché); } \\
\text { tout cela s'applique à l'ensemble du pays. }\end{array}$ \\
\hline Calcium & $33,3 \%$ & $\begin{array}{l}\text { - Promouvoir la production et la transformation nationales du sésame pour approvi- } \\
\text { sionner les marchés intérieurs, en commençant par les régions actuellement productrices } \\
\text { de sésame. Le sésame est très riche en calcium, mais il est principalement exporté vers les } \\
\text { pays étrangers. }\end{array}$ \\
\hline Vitamine A & $35,4 \%$ & $\begin{array}{l}\text { - Augmenter la production horticole (riche en vitamine A) dans les régions présentant } \\
\text { des conditions biophysiques appropriées (en particulier dans le Sud-Ouest du pays). } \\
\text { - Distribuer des produits horticoles aux régions ne présentant pas des conditions agroé- } \\
\text { cologiques appropriées (surtout dans le Nord). }\end{array}$ \\
\hline $\begin{array}{l}\text { Fer, Zinc, } \\
\text { Folate }\end{array}$ & $62,5-79,1 \%$ & $\begin{array}{l}\text { - Réduire la « perte en nutriments » des céréales et des légumineuses qui se produit } \\
\text { entre les sites de production et les marchés. } \\
\text { - Les obstacles en matière de nutrition pour ces nutriments dessinent un schéma spatial si- } \\
\text { milaire dans tout le pays (voir les cartes pour référence). }\end{array}$ \\
\hline $\begin{array}{l}\text { Tous les } \\
\text { nutriments }\end{array}$ & --- & $\begin{array}{l}\text { - Mettre en œuvre des programmes de protection sociale à court terme pour garantir } \\
\text { un apport minimal en nutriments essentiels aux ménages les plus sous-alimentés, dont une } \\
\text { grande partie vit à Ouagadougou (Centre). }\end{array}$ \\
\hline
\end{tabular}




\section{Chapitre 4}

\section{CARTOGRAPHIE DE L'ADÉQUATION RELATIVE DES INFRASTRUCTURES D'ÉLEVAGE POUR UNE MEILLEURE ORIENTATION DES POLITIQUES D'INVESTISSEMENT}

\subsection{INTRODUCTION}

L'élevage offre toute une série d'avantages aux habitants des pays en développement. Tout d'abord, il permet aux populations de conserver une alimentation plus nutritive et plus diversifiée (notamment pour éviter les carences en vitamine B12 et en calcium, voir le chapitre 3). L'élevage est également une source importante de revenus, grâce à la vente de produits d'origine animale tels que le lait, les œufs ou la laine. Enfin, il fournit du fumier (engrais organique), des services de tracteur et de labourage, des fonctions d'assurance et un statut social. Pour ces raisons, la plupart des ménages ruraux en Afrique essaient de combiner l'agriculture avec l'élevage de petits ou de grands animaux.

Le Tableau 4.1. présente les indicateurs du secteur de l'élevage au Burkina Faso, dans les pays voisins et dans l'ensemble de I'Afrique de l'Ouest pour l'année 2016. Le cheptel du Burkina Faso est l'un des plus importants d'Afrique de I'Ouest, avec environ 10 millions de bovins, 15 millions de caprins et 10 millions d'ovins. Le Niger enregistre des chiffres similaires pour ces trois types de bétail, tout comme le Mali pour les bovins. La densité du bétail est plus élevée au Burkina Faso que dans les pays voisins $(0,70$ unité de bétail par hectare de terre agricole contre seulement 0,27 dans l'ensemble de l'Afrique de l'Ouest). Toutefois, la contribution du bétail au PIB agricole du Burkina Faso est nettement inférieure à celle du Mali et du Niger ( $22 \%$ contre $28 \%$ et $27 \%$ respectivement). Le sous-secteur de l'élevage au Burkina Faso souffre d'une faible productivité.

\section{Mots clés}

\section{Unités de bétail tropicales}

(UBT) : Unités de mesure utilisées pour exprimer les nombres agrégés de différents types d'animaux.

Inter- et extrapolation : Technique permettant de déduire une valeur à partir d'observations réelles adjacentes.

\section{Indice d'adéquation relative :}

Un indice utilisé pour comparer l'adéquation d'une chose (telle que l'infrastructure du bétail) avec une autre chose connexe (telle que les animaux d'élevage).

Tableau 4.1. Indicateur's clés surr le secteur' de l'élevage, Afrique de l'Ouest (2016)

\begin{tabular}{|c|c|c|c|c|c|}
\hline Pays & $\begin{array}{l}\text { Bovins } \\
\text { (unité) }\end{array}$ & $\begin{array}{l}\text { Chèvres } \\
\text { (unité) }\end{array}$ & $\begin{array}{l}\text { Moutons } \\
\text { (unité) }\end{array}$ & $\begin{array}{c}\text { Densité du bétail } \\
\text { (UBT par hectare } \\
\text { agr. ) }\end{array}$ & $\begin{array}{c}\text { Part de I'élevage } \\
\text { (\% du PIB agr.) }\end{array}$ \\
\hline Burkina Faso & 9.457 .934 & 14.737.393 & 9.842 .712 & 0,70 & $22,2 \%$ \\
\hline Mali & 10.941 .300 & 22.141 .497 & 15.900 .300 & 0,27 & $28,4 \%$ \\
\hline Niger & 12.783 .548 & 16.098 .058 & 11.899 .263 & 0,25 & $27,1 \%$ \\
\hline Bénin & 2.339 .000 & 1.836 .000 & 1.836 .000 & 0,49 & $7,9 \%$ \\
\hline Togo & 441.662 & 3.000 .000 & 2.347 .424 & 0,30 & $16,1 \%$ \\
\hline Ghana & 1.734 .000 & 6.352 .000 & 4.522 .000 & 0,18 & $6,3 \%$ \\
\hline Côte d'Ivoire & 1.674 .511 & 1.441 .506 & 1.815 .495 & 0,09 & $8,3 \%$ \\
\hline Afrique de l'Ouest & 74.110 .240 & 158.209 .139 & 111.802 .731 & 0,27 & $12,6 \%$ \\
\hline
\end{tabular}

Notes : L'UBT « Unité de bétail tropical » équivaut à $250 \mathrm{~kg}$ de poids vif et facilite l'agrégation entre les différents types d'animaux.

Source : Les Auteurs, à partir de FAOSTAT (Version du 10 septembre 2019).

La Figure 4.1. montre que la part des dépenses d'élevage dans les dépenses agricoles totales est inférieure à $10 \%$ sur la période 2004-2016. Elle montre également que la contribution de l'élevage au PIB agricole a diminué au fil des ans (de $36 \%$ en 2004 à $22 \%$ en 2016). Toutefois, la contribution au PIB reste nettement supérieure à ce que le sous-secteur de l'élevage reçoit proportionnellement en termes de dépenses agricoles totales.

Outre son importante contribution à la richesse du pays, l'augmentation des investissements dans l'élevage est essentielle pour résoudre le problème de la faible productivité et pour préparer l'avenir. Alors que la demande de produits de l'élevage a commencé à stagner dans plusieurs pays industrialisés (en raison de problèmes d'environnement, de santé et de bien-être animal), la révolution de l'élevage n'a pas encore eu lieu dans la plupart des pays d'Afrique subsaharienne (Gerber et al. 2010:xi). Au Burkina Faso, la population devrait exploser, passant de 18 millions d'habitants en 2016 à 45 millions en 2050, la moitié de la population vivant dans les zones urbaines. Les économistes estiment que le PIB triplera d'ici 2050, pour atteindre près de 2000 USD par 
habitant. Ces prévisions pourraient entraîner une croissance de près de 300\% de la demande de produits d'origine animale (FAO, 2019).

Figure 4.1. Évolution économique et budgétaire de l'élevage dans le secteur agricole, Burkina Faso (2004-2016)

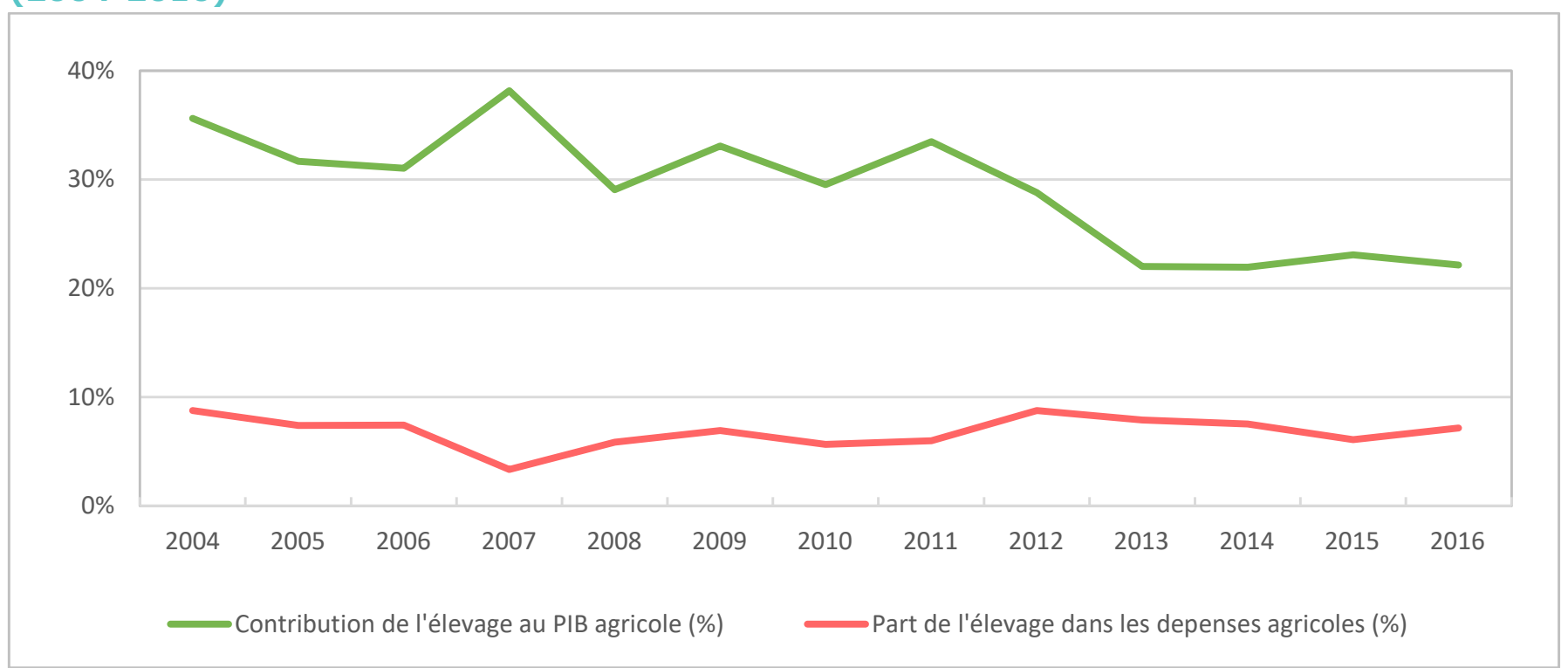

Source : Les Auteurs, à partir des données FAOSTAT (version du 10 septembre 2019) et revue des dépenses publiques /SPCPSA.

Il est évident qu'une telle révolution ne se fera pas sans heurts et entraînera de nombreux défis. Tout d'abord, les systèmes de production animale devront devenir plus efficaces, tant sur le plan économique que sur le plan environnemental. Deuxièmement, à mesure que les chaînes de valeur des produits de l'élevage deviennent plus complexes, elles exigeront de porter une plus grande attention aux normes et réglementations en matière de sécurité alimentaire. Enfin, la production et la transformation des produits de l'élevage se concentreront probablement autour des principaux centres de consommation, au détriment des zones rurales ou éloignées. Ces facteurs pourraient conduire à une répartition inégale des avantages et des opportunités offerts par la croissance du sous-secteur de l'élevage au sein des différentes couches de la société burkinabé. (FAO, 2019; Gerber et al., 2010).

Ce chapitre vise à identifier et à localiser l'adéquation relative des infrastructures d'élevage au Burkina Faso afin de mieux orienter les investissements. L'objectif final est de contribuer à élaborer des politiques mieux ciblées en proposant des options pour réduire les disparités spatiales dans le pays. Dans la première partie, nous discutons de la qualité des données et des informations disponibles, lesquelles seront utilisées pour mieux comprendre la méthodologie adoptée pour déterminer l'adéquation des infrastructures d'élevage. Ensuite, nous comparons les profils obtenus pour six types d'infrastructures d'élevage avec les politiques correspondantes dans toutes les provinces.

\subsection{DONNEES SUR L'ELEVAGE ET LES INFRASTRUCTURES D'ELEVAGE}

En Afrique subsaharienne et au Burkina Faso en particulier, les données fiables sur le nombre de têtes de bétail sont rares. Le nombre de têtes de bétail est souvent obtenu par des procédures d'estimation (c'est-à-dire par inter- ou extrapolation), comme le montrent les taux de croissance constants entre les recensements. Par exemple, nous constatons des taux de croissance fixes de 4,7\% pour le bétail au cours de la période 1990-1999 et de 2,0\% pour la période 2003-2011. Pour les ovins et les caprins, la stabilité de la croissance du bétail est encore plus frappante : environ 3,0\% pour toute la période 1990-2011 (Pica-Ciamarra et al. 2016:95). Étant donné l'insécurité accrue dans le Nord du Burkina Faso et son impact probable sur la propriété des animaux (Ollo, Ouedraogo, Degueurse, Ouattara, \& Hitayezu, 2013), de profonds écarts entre les statistiques et la réalité peuvent exister.

Au Burkina Faso, le dernier recensement agricole général remonte à 2006-2010, avec des données sur le bétail obtenues entre janvier 2008 et janvier 2009 auprès de 7500 ménages (Pica-Ciamarra et al., 2016). En 2015, avec les perturbations politiques liées au changement de pouvoir, un nouveau recensement initialement prévu en 2017 a été reporté. ${ }^{8}$ En dehors des recensements assez irréguliers, le pays mène chaque année une enquête permanente agricole (EPA). Malgré la qualité douteuse de certaines statistiques, I'EPA est une source importante d'informations sur l'agriculture et l'élevage.

Compte tenu de l'objectif de cette analyse, nous sommes principalement intéressés par les données relatives au nombre de têtes de bétail et aux infrastructures. Pour le cheptel total, nous nous appuyons sur les estimations produites par le ministère des

${ }^{8}$ Voir le Programme mondial de recensement de l'agriculture (http://www.fao.org/world-census-agriculture/wcarounds/results/en). 
Ressources animales et de la Pêche (MRAH) pour les 45 provinces du pays. Ces données couvrent neuf types d'animaux (ânes, bovins, chameaux, chèvres, chevaux, moutons, pintades, porcs et volailles) sur la période 2013-2019. Tout comme les observations ci-dessus, ces chiffres récents sont basés sur des extrapolations utilisant les taux de reproduction et d'exploitation pour chaque type d'animal ; ils ne sont pas dérivés des données primaires.

Le Tableau 4.2. donne un aperçu non exhaustif des différents types d'infrastructures. Selon le type et la source des données, l'année de référence et la couverture spatiale varient (là encore, les différentes sources sont de qualité douteuse). Par exemple, la Direction Générale des Etudes et des Statistiques Sectorielles (DGESS) du MRAH fournit des informations sur les points d'eau en 2019, mais seulement pour 10 provinces. La Direction Générale des Espaces et des Aménagements Pastoraux (DGEAP) assure une couverture plus large des zones pastorales, couvrant 33 provinces, mais elle n'est pas récente (2009). Pour les services vétérinaires, les quais et parcs de vaccination, les pistes, les abattoirs, les boucheries, les séchoirs, les marchés et les magasins de produits d'élevage, la couverture spatiale est également faible. La dernière ligne du Tableau 4.2. est un exercice d'harmonisation (considéré comme un recensement) mené par le MRAH qui comprend 30 types d'infrastructures pour le bétail. Ce recensement couvre les 45 provinces du pays et inclut les coordonnées GPS pour l'année 2016. Toutefois, il ne contient pas d'informations sur les zones pastorales et ne fournit pas de détails sur les capacités ou les fonctionnalités de chaque infrastructure. En comparant ce recensement à d'autres sources, nous constatons plusieurs dissemblances. Compte tenu de sa couverture spatiale complète et précise, et après validation par les experts du pays lors d'un atelier tenu du 10 au 12 septembre 2019 à Bobo Dioulasso, notre analyse s'appuie principalement sur ce recensement de 2016 et le relie aux effectifs du bétail de 2016.

Tableau 4.2. Aperçu des données existantes sur les infrastructures d'élevage, Burkina Faso (200919)

\begin{tabular}{|l|c|c|}
\hline Types d'infrastructure & Source & $\begin{array}{c}\text { Année } \\
\text { provinces }\end{array}$ \\
\hline Points d'eau & DGESS/MRAH & 2019 \\
\hline Zones pastorales & DGEAP/MRAH & $2009 *$ \\
\hline Services vétérinaires & CEFCOD & 2013 \\
\hline Quais de vaccination et parcs a bétail & DGESS/MRAH & 2018 provinces \\
\hline Pistes & DGESS/MRAH & 2017 \\
\hline $\begin{array}{l}\text { Abattoirs, boucheries, séchoirs, marchés et magasins de bé- } \\
\text { tail }\end{array}$ & DGESS/MRAH & 2018 \\
\hline « Recensement 》 de 30 types d'infrastructure d'élevage & MRAH & 2016 \\
\hline
\end{tabular}

Note : * Années des dernières specifications.

Source : Les Auteurs, en utilisant les différentes sources de données mentionnées dans le tableau.

La Figure 4.2. montre la répartition spatiale des infrastructures d'élevage selon le recensement du MRAH en 2016. Les parcs de vaccination (plus de 400) sont les plus courants et sont concentrés dans les provinces de Zoundwéogo et de Sourou, respectivement dans le Centre-Sud et le Centre-Nord du pays. Nous identifions plus de 300 forages au niveau national, principalement dans la partie Sud-Ouest du pays. A l'exception des zones d'abattage, toutes les autres infrastructures recensées en 2016 sont nettement moins nombreuses et ne dépassent pas 50 unités au total. 


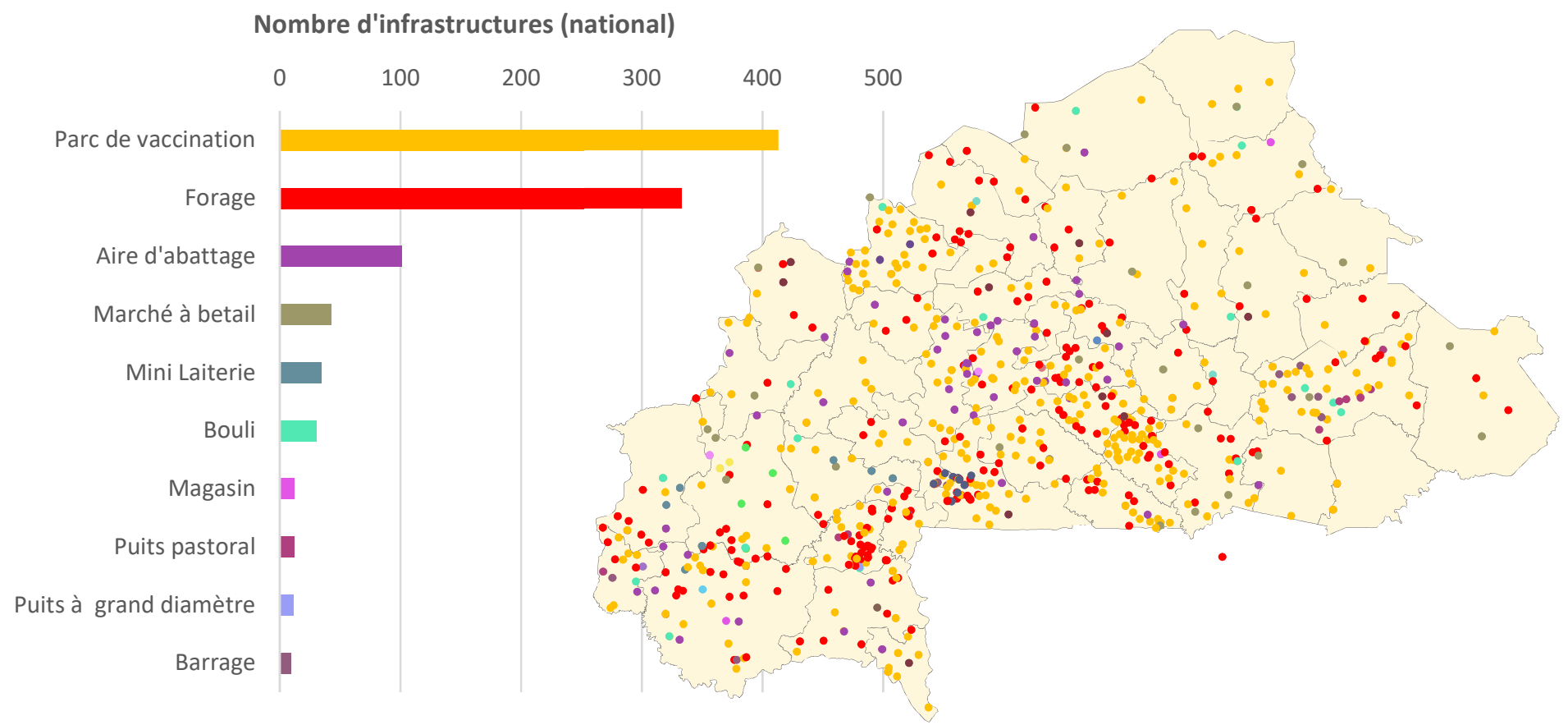

Note : Une inspection approfondie a montré que la plupart des forages de la province de Houet étaient mal référencés et situés par erreur dans la province de Bougouriba.

Source : Les Auteurs, sur la base du resencement du MRAH en 2016.

\subsection{ADEQUATION DES INFRASTRUCTURES D"ELEVAGE}

Afin de mesurer l'adéquation des infrastructures d'élevage, nous utilisons un indice d'adéquation relative (IAR) que nous appliquons aux 45 provinces du pays. La relativité de cet indice est triple. Premièrement, I'IAR présente l'adéquation des infrastructures par rapport à la taille du cheptel ; la capacité des infrastructures d'élevage doit généralement refléter la taille du cheptel. Ensuite, nous normalisons I'IAR en utilisant la relation moyenne entre les infrastructures d'élevage et la population telle qu'elle est observée ailleurs au Burkina Faso. En tant que tel, I'IAR ne garantit pas une adéquation absolue. Pour mieux cibler et réduire les éventuelles disparités géographiques, il est cependant essentiel de pouvoir distinguer les différents niveaux d'adéquation. Troisièmement, en l'absence d'informations sur la capacité de chaque infrastructure, nous supposons simplement que la capacité totale est plus élevée là où le nombre d'infrastructures est plus élevé, sans connaître les différences exactes en termes de capacité. Pour les infrastructures de pâturage, nous exprimons la capacité en termes de superficie disponible, l'adéquation relative étant basée sur la superficie totale (fonctionnelle et potentielle), au lieu de classer les infrastructures. La Figure 4.3. fournit une illustration conceptuelle qui soutend I'IAR. La logique ici est que I'adéquation relative des infrastructures d'élevage existe si les classements de la taille du cheptel et de la dotation en infrastructures des provinces s'alignent. Si la dotation en infrastructures correspond à la moyenne observée dans d'autres provinces ayant un nombre d'animaux similaire, I'IAR s'approchera de $100 \%$. Cependant, si une province est moins dotée en infrastructures alors qu'elle possede un cheptel important, nous la caractérisons comme relativement sousdotée en infrastructures et I'IAR approchera $0 \%$. Un nombre relativement faible d'animaux et un nombre relativement élevé d'infrastructures indiquent une surdotation relative en infrastructures d'élevage, et I'IAR tendra vers $200 \%$ (voire plus). ${ }^{9}$

\footnotetext{
${ }^{9}$ En effet, l'indice d'adéquation relatif peut dépasser 200\%, ce qui est lié au choix d'avoir une normalisation basée sur la moyenne (voir formule correspondante). En outre, étant donné que plusieurs provinces peuvent occuper le même rang, la ligne des $100 \%$ n'est pas nécessairement égale à la ligne des 45 degrés.
} 


\section{Figure 4.3. Illustration conceptuelle de l'indice d'adéquation relative (IAR)}

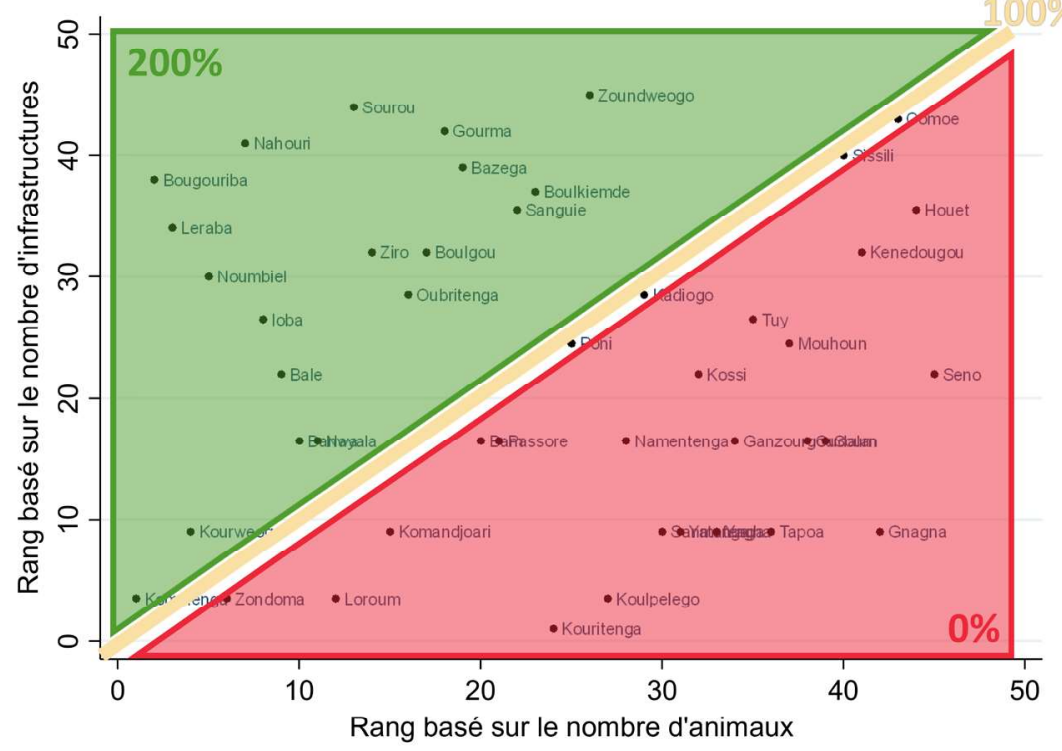

\begin{tabular}{|l|l|}
\hline IAR & Interprétation \\
\hline$<100 \%$ & Relativement sous-dotée en infrastructures \\
\hline$\approx 100 \%$ & $\begin{array}{l}\text { Des infrastructures en ligne avec la moyenne } \\
\text { observée dans les provinces similaires }\end{array}$ \\
\hline$>100 \%$ & Relativement sur-dotée en infrastructures \\
\hline
\end{tabular}

Source : Les Auteurs.

Nous présentons ci-dessous la formule exacte pour calculer I'IAR. Nous utilisons la procédure de normalisation habituelle, de sorte que la différence moyenne entre les deux classements soit égale à 100\%. Pour les infrastructures de pâturage, nous remplaçons les différences par des ratios, qui indiquent le nombre d'hectares de pâturages par animal. Hormis cette modification, la logique et l'interprétation restent inchangées.

$$
\text { Indice d'Adequation Relative }(I A R)=\frac{\operatorname{diff}(\operatorname{rang})-\min (\operatorname{diff}(\operatorname{rang}))}{\text { moyenne }(\operatorname{diff}(\operatorname{rang}))-\min (\operatorname{diff}(\mathrm{rang}))} \times 100
$$

Pour calculer ces indices d'adéquation relative, nous faisons les choix méthodologiques suivants. Pour les données compilées dans le cadre du recensement de 2016, nous regroupons (en additionnant les chiffres observés) 18 types d'infrastructures en cinq catégories en fonction du besoin auquel elles répondent (voir Tableau 4.3.). Nous distinguons les infrastructures d'abreuvement, de santé, de vente, de production de viande et de production de lait. Les autres types d'infrastructures disponibles dans le cadre du recensement sont considérés comme moins appropriés ou moins fiables. Afin de s'aligner sur les données relatives au nombre de têtes de bétail, qui ne sont disponibles qu'au niveau provincial, nous additionnons le nombre total d'infrastructures (dans les cinq catégories) dans chacune des 45 provinces, en ignorant leur emplacement précis. Pour les infrastructures de pâturage, nous considérons la superficie totale des zones pastorales fonctionnelles ajoutée à la superficie totale des terres pastorales potentielles. L'indice qui en résulte montre l'adéquation relative des pâturages, y compris les zones de pâturage potentielles.

Tableau 4.3. Catégories d'infrastructures, Burkina Faso (2009/2016)

\begin{tabular}{|c|c|c|c|}
\hline & Catégories & Sous-catégories & Source \\
\hline 1 & Infrastructures d'abreuvement & $\begin{array}{l}\text { Abreuvoirs, barrages, forages, puits, puits pastoraux, puits de grand dia- } \\
\text { mètre, réservoirs d'eau, boulis }\end{array}$ & \multirow[t]{5}{*}{$\begin{array}{l}\text { MRAH recense- } \\
\text { ment (2016) }\end{array}$} \\
\hline 2 & Infrastructures de santé & Parcs de quarantaine, parcs de vaccination, stations vétérinaires & \\
\hline 3 & Infrastructures de vente & Marchés aux bestiaux, quai de chargement & \\
\hline 4 & $\begin{array}{l}\text { Infrastructures de production } \\
\text { de viande }\end{array}$ & Abattoirs, zones d'abattage, boucheries & \\
\hline 5 & $\begin{array}{l}\text { Infrastructures de production } \\
\text { de lait }\end{array}$ & Laiteries, mini-laiteries & \\
\hline 6 & Infrastructures de pâturage & Zones pastorales fonctionnelles, zones pastorales potentielles & $\begin{array}{l}\text { DGEAP/MRAH } \\
(2009)\end{array}$ \\
\hline
\end{tabular}

Source : Les Auteurs, sur la base des données DGEAP/MRAH (2009) et du recensement du MRAH (2016).

Cette analyse englobe tous les grands et petits ruminants, tels que les bovins, les ânes, les chevaux, les chameaux, les chèvres et les moutons. Étant donné l'année du recensement des infrastructures d'élevage, nous nous limitons à 2016, en supposant que l'étendue des zones pastorales fonctionnelles et potentielles est restée stable depuis 2009. Les unités de bétail tropicales (UBT) sont utilisées pour agréger les nombres de différents types d'animaux ; une UBT correspond aux besoins en fourrage d'une 
vache laitière adulte (FAO, 2011). Les coefficients UBT pour chaque type de bétail diffèrent d'une région à I'autre. Pour I'Afrique subsaharienne, les chèvres et les moutons représentent 10\% de I'UBT, 50\% pour les bovins et les chevaux, 30\% pour les ânes et $70 \%$ pour les chameaux (FAO 2011:37). En appliquant ces coefficients aux chiffres réels (tels que résumés dans le tableau cidessous), la Figure 4.4. montre la répartition spatiale des grands et des petits ruminants exprimée en UBT au Burkina Faso en 2016. L'analyse révèle un nombre important de ruminants dans tout le pays. Cependant, certaines provinces sont particulièrement concentrées, notamment Seno et Gnagna dans le Nord-Est du pays et les provinces de Houet, Comoé et Sissili dans le Sud-Ouest. Ensemble, ces cinq provinces représentent près d'un tiers des ruminants en équivalent UBT. Toutefois, les provinces qui comptent un nombre de ruminants nettement inférieur sont dispersées dans tout le pays.

\section{Figure 4.4. Distribution spatiale des grands et des petits ruminants en UBT, Burkina Faso (2016)}

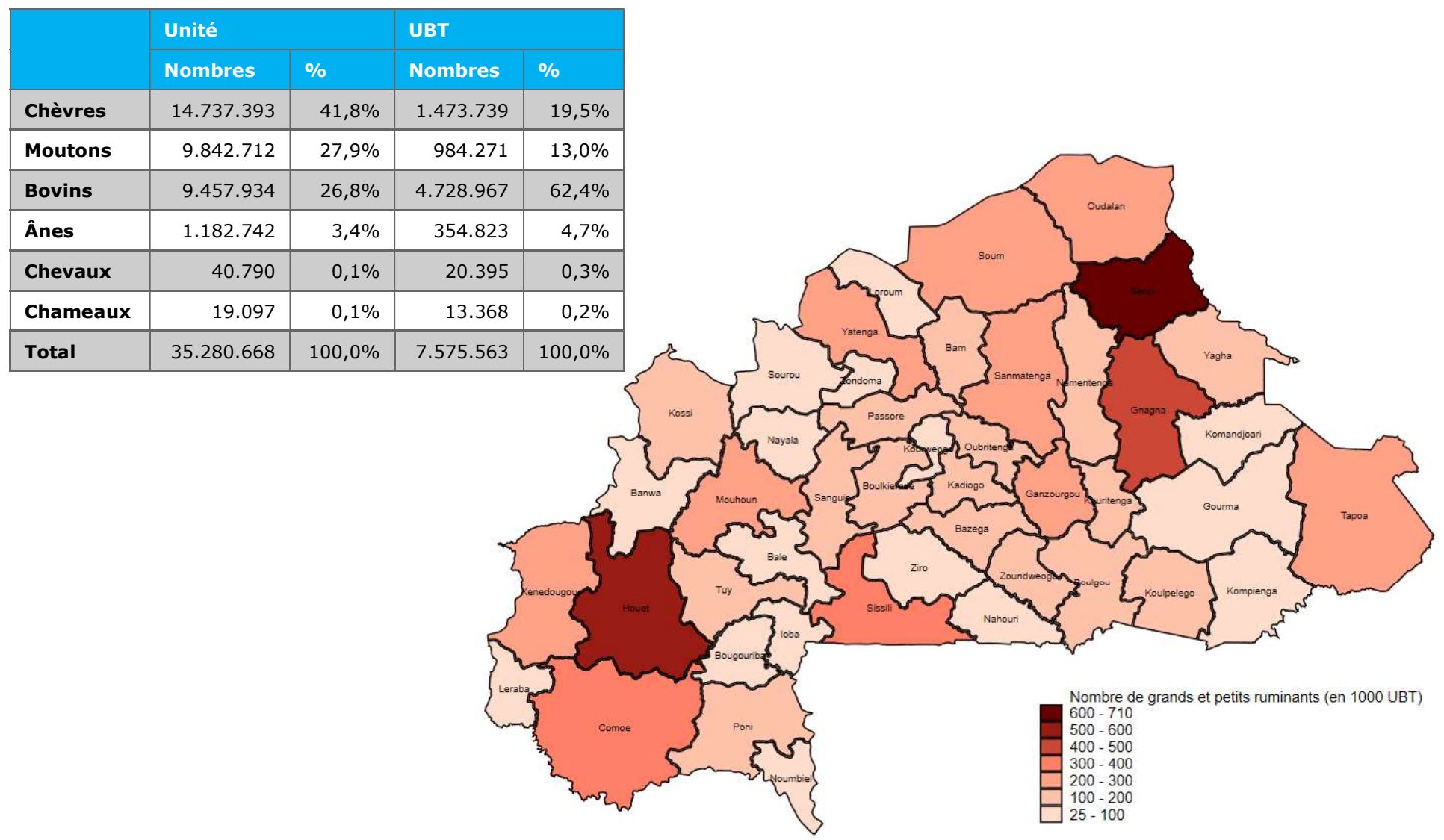

Source : Les Auteurs, sur la base des données du MRAH (2016) et de la FAO (2011). 


\subsection{RESULTATS}

Quel est le rapport entre la répartition des ruminants et la densité géographique des infrastructures d'élevage dans le pays ? Pour répondre à cette question clé, nous présentons deux cartes pour chaque catégorie d'infrastructure. La première carte montre la répartition des infrastructures en chiffres absolus et la seconde montre l'adéquation relative en utilisant I'IAR. La seconde carte reflète l'interaction entre la première carte et la carte qui décrit la distribution des ruminants en UBT (c'est-à-dire la Figure 4.4.).

\section{Infrastructures d'abreuvement}

La Figure 4.5. montre la répartition spatiale des infrastructures d'abreuvement en chiffres absolus et en adéquation relative. Ce qui ressort immédiatement, c'est la forte concentration de ce type d'infrastructures dans quelques provinces du Sud-Ouest du pays, à savoir Houet, Comoé et Kénédougou. Ces provinces abritent également un grand nombre de ruminants. Dans ce cas, la fourniture d'infrastructures est dite « en ligne », avec un indice d'adéquation relatif d'environ $100 \%$. Cependant, le Nord-Est autour de la province de Seno, qui se caractérise également par une forte prévalence de ruminants, manque clairement d'infrastructures d'abreuvement. Ceci est confirmé par I'IAR, qui se situe à moins de $60 \%$ pour la plupart des provinces du Nord-Est. A l'inverse, trois provinces - Léraba, Bougouriba et Nahouri - présentent une offre relativement excédentaire, principalement en raison de la faible concentration de ruminants.

\section{Figure 4.5. Nombre et adéquation relative des infrastructures d'abreuvement, Burkina Faso (2016)}
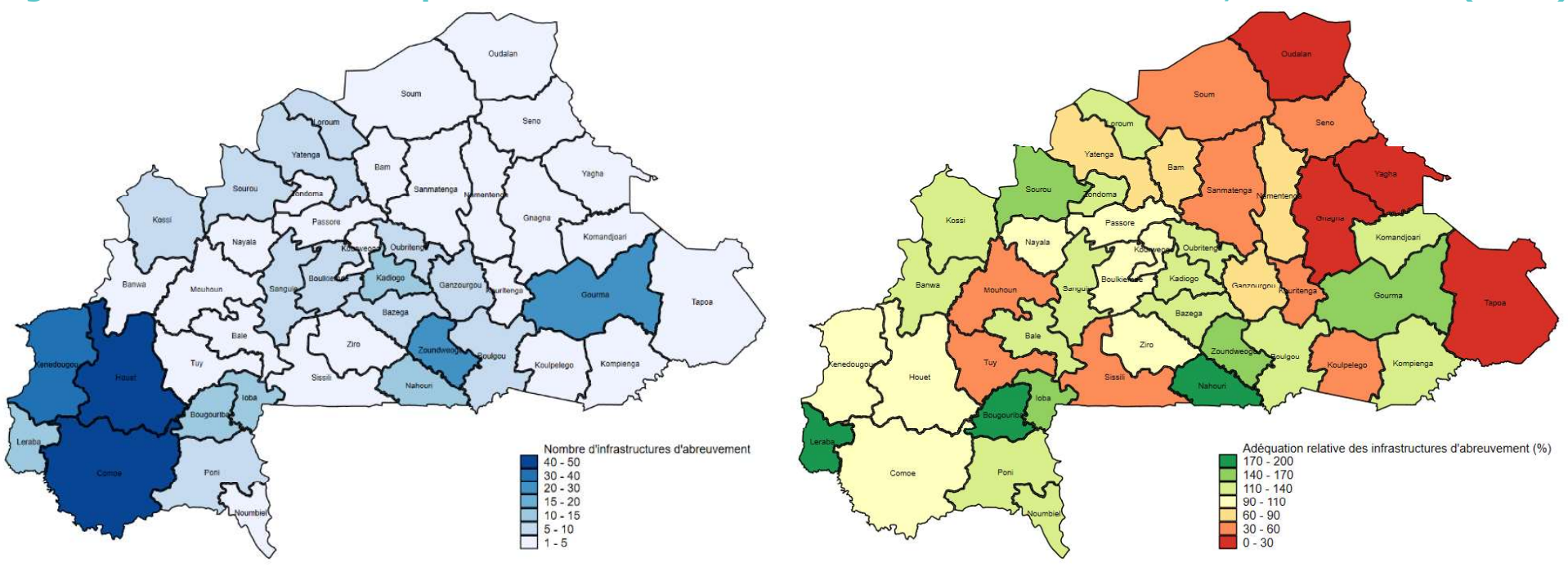

Source : Les Auteurs, sur la base des données du MRAH (2016).

\section{Infrastructures de santé}

La Figure 4.6. montre les infrastructures sanitaires du bétail telles que les services vétérinaires. Le Sud-Ouest du Burkina Faso est mieux doté que le Nord-Est du pays, où I'IAR se situe en dessous de $60 \%$ dans plusieurs provinces. Dans le Sud-Ouest, la dotation en infrastructures correspond mieux à la concentration de ruminants à Comoé, Sissili et Tuy, où l'IAR est proche de $100 \%$ dans chaque province. Dans cette même région du pays, on constate même une forte surdotation relative pour les provinces de Léraba, Bougouriba et Noumbiel. Plus au centre, on observe également que la forte concentration en infrastructures sanitaires à Zoundwéogo, Gourma et Sourou dépasse relativement la faible concentration en ruminants. 

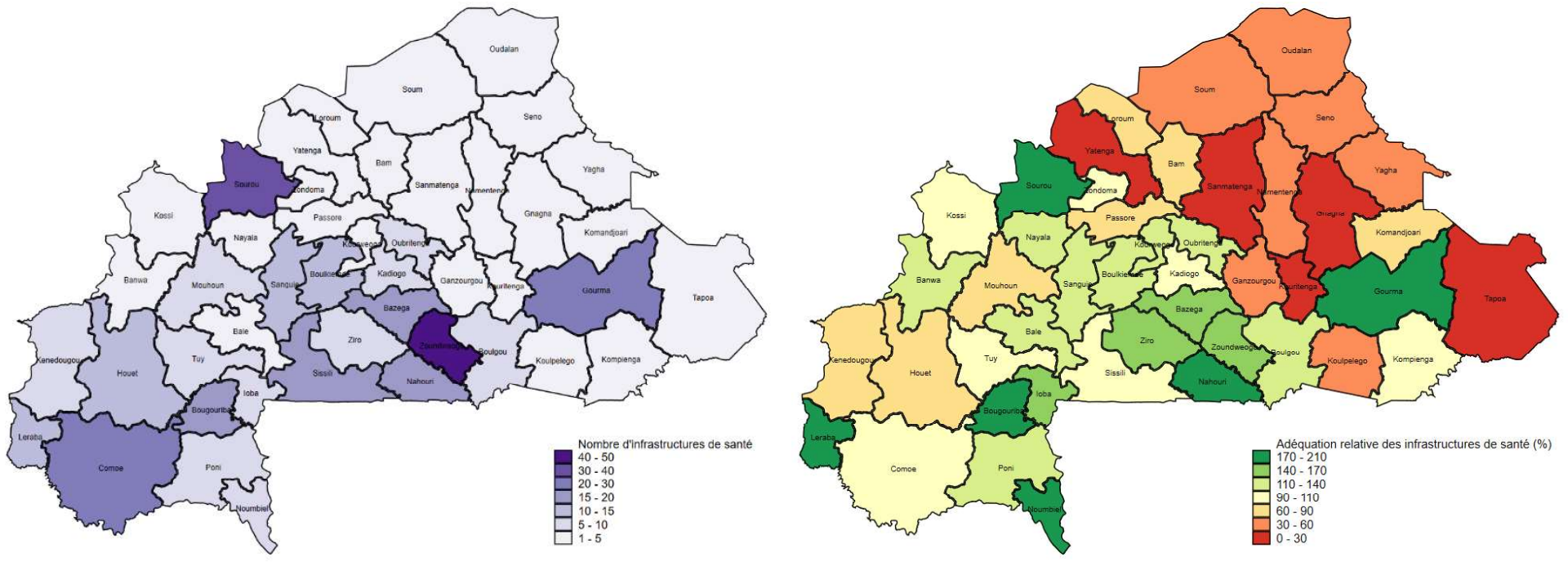

Source : Les Auteurs, sur la base des données du MRAH (2016).

\section{Infrastructures de vente}

Les infrastructures de vente présentent une distribution moins contrastée dans l'espace que les infrastructures d'abreuvement et de santé (voir Figure 4.7.). Plusieurs provinces du Nord-Est, telles que Gnagna et Soum, sont relativement mieux dotées en infrastructures de vente. Dans le centre du pays, Mouhoun et Boulkiemdé sont relativement sous-dotées, avec un IAR inférieur à $30 \%$. Les infrastructures de vente correspondent à la forte concentration de ruminants à Houet et Comoé, tandis que Séno, dans le Nord-Est, est relativement moins bien dotée, avec un indice inférieur à $30 \%$.

\section{Figure 4.7. Nombre et adéquation relative des infrastructures de vente, Burkina Faso (2016)}
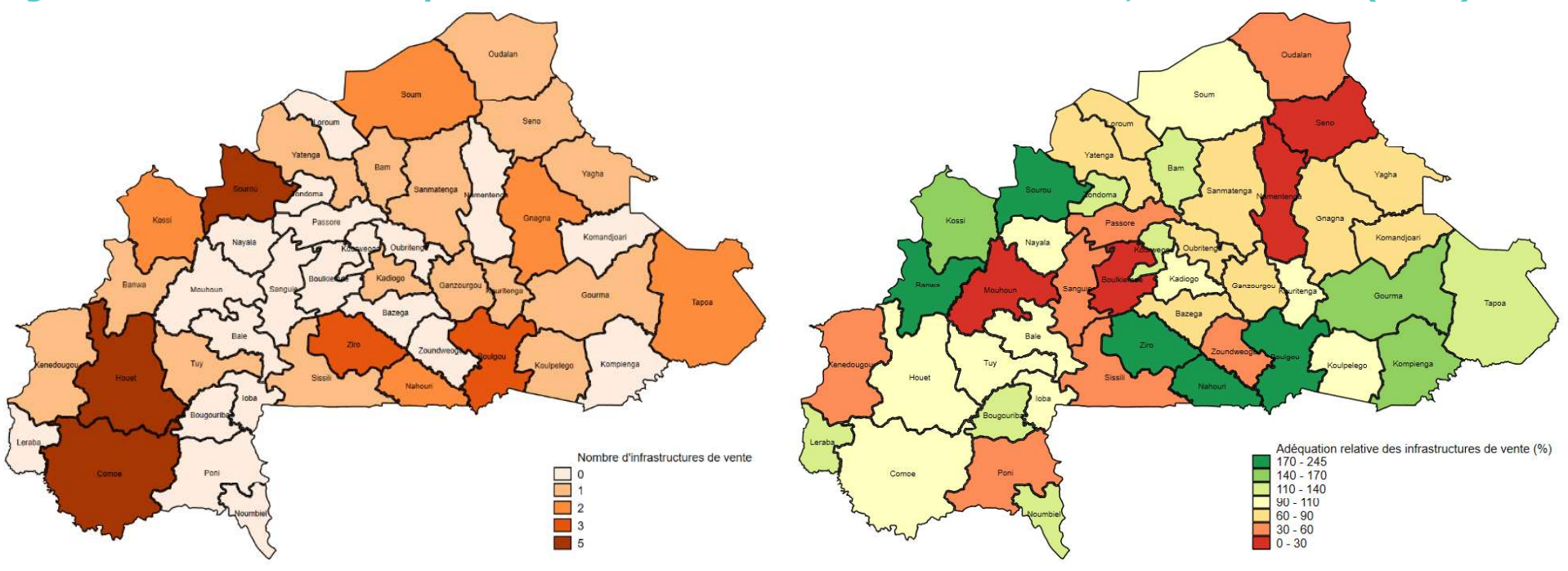

Source : Les Auteurs, sur la base des données du MRAH (2016).

\section{Infrastructures de production de viande}

La Figure 4.8. reprend les schémas précédents entre les régions du Nord-Est et du Sud-Ouest du Burkina Faso. La plupart des abattoirs, des zones d'abattage et des boucheries sont situés dans le Sud-Ouest, avec de fortes concentrations à Sanguié et à Comoé, tandis que la plupart des provinces du Nord-Est ne comptent qu'une infrastructure au maximum. Nous résumons l'adéquation relative de la même manière, en superposant les informations sur la distribution des ruminants avec les infrastructures de production de viande.

Nous pouvons contraster les fortes concentrations de ruminants dans les provinces de Houet et de Comoé (qui disposent d'infrastructures suffisantes) avec Seno et Gnagna où la densité élevée de ruminants n'est pas soutenue par des infrastructures comme le reflète I'IAR en dessous de $30 \%$. On observe également une relative sous-dotation dans toutes les provinces du Nord-Est et à Tapoa. 

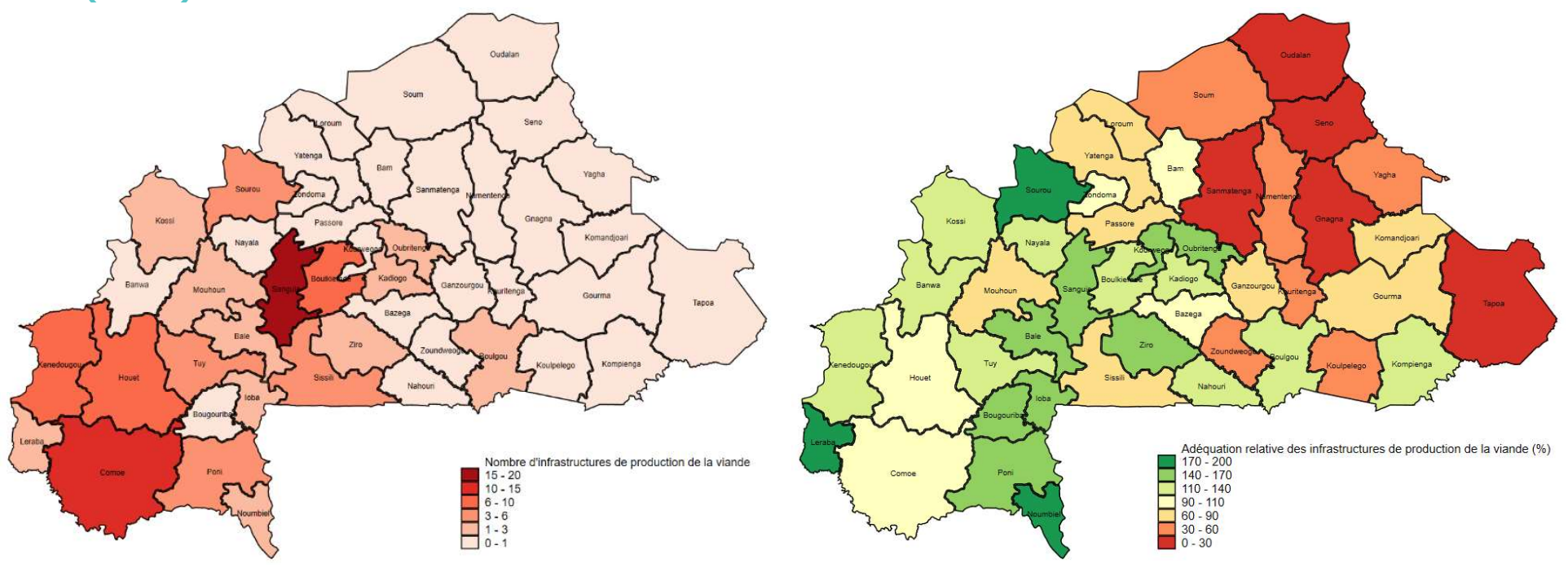

Source : Les Auteurs, sur la base des données du MRAH (2016).

\section{Infrastructures de production de lait}

La Figure 4.9. montre la répartition des petites et grandes laiteries en nombre absolu et en adéquation relative. Là encore, une tendance similaire se dégage, avec une meilleure dotation dans le Sud-Ouest, en particulier dans les provinces de Kénédougou, Houet, Comoé et Tuy, par rapport au reste du pays. La carte de I'IAR montre une répartition des infrastructures bien adaptée à Houet et Comoé et même un excédent à Kénédougou et Tuy, en fonction du nombre relatif de têtes de bétail. L'indice est bien inférieur à $60 \%$ pour la majorité des provinces du Nord-Est qui ont une forte présence de ruminants. 

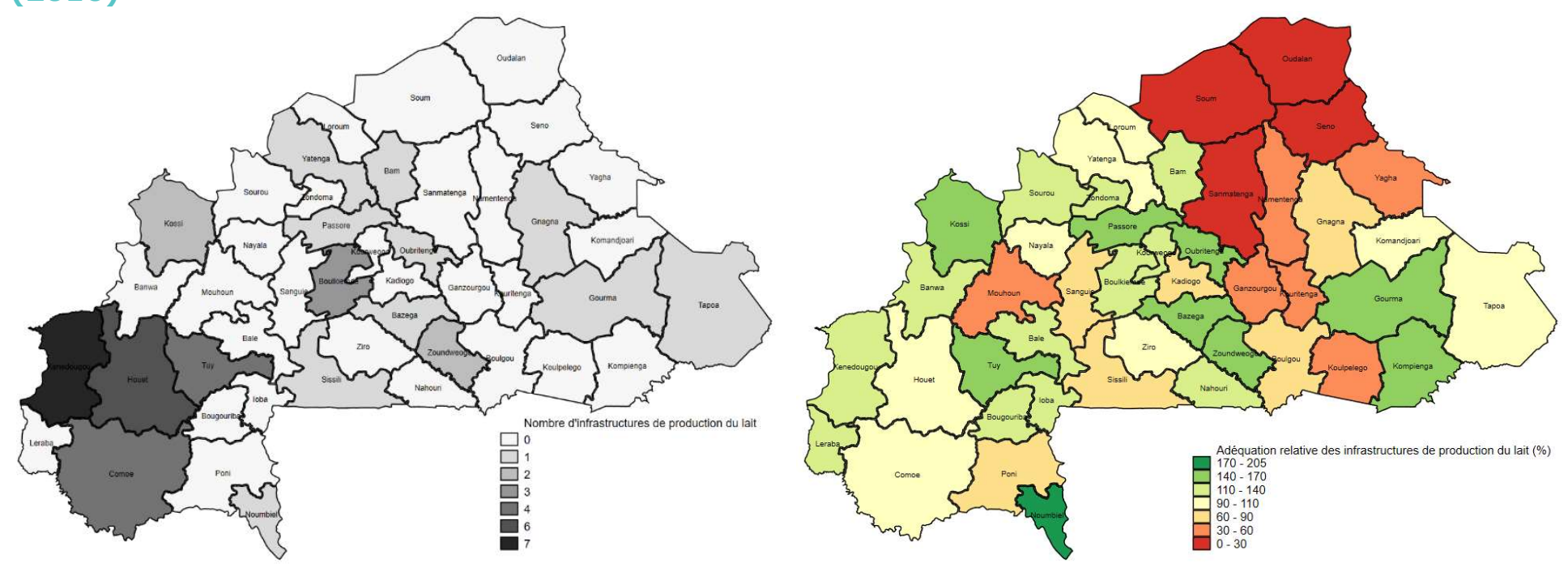

Source : Les Auteurs, sur la base des données du MRAH (2016).

\section{Infrastructures de pâturage existantes et potentielles}

La Figure 4.10. montre la répartition des zones de pâturage en hectares et leur adéquation relative, y compris tous les pâturages potentiels identifiés précédemment. Contrairement aux cartes précédentes, les provinces de l'Est et du Nord-Est comme Gourma, Oudalan et Gnagna, qui enregistrent plus de 240000 hectares de zones pastorales, obtiennent de meilleurs résultats, ce qui se reflète également dans leur IAR élevé. Les provinces les plus sous-dotées sont situées dans le Sud et le Sud-Ouest du pays.

Figure 4:10. Nombre et adéquation relative des înfrastructures de pâturage existantes et potentielles, Burkina Faso (2016)
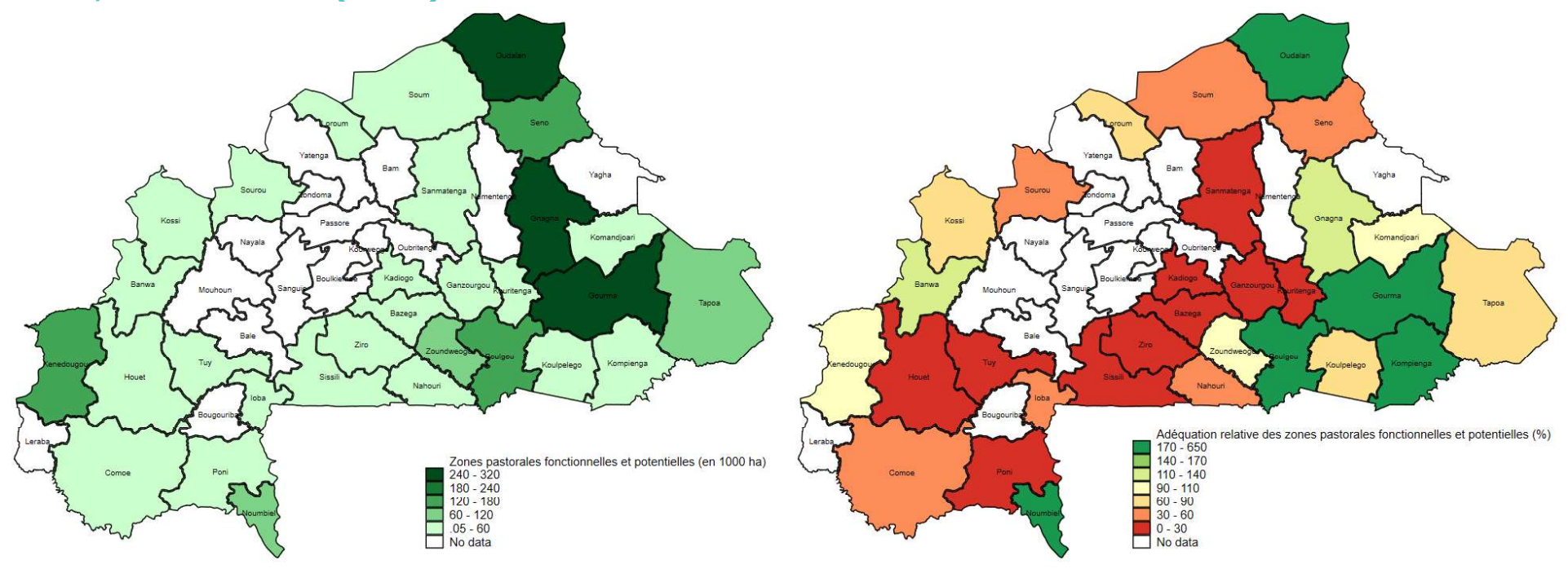

Source : Les Auteurs, sur la base des données du MRAH (2016).

\subsection{VERS UNE POLITIQUE D'INVESTISSEMENT PLUS CIBLEE SUR LE PLAN GEO- GRAPHIQUE}

Cette section utilise les résultats de l'adéquation relative pour suggérer des domaines d'intervention prioritaires pour les infrastructures d'élevage. La Politique nationale de développement durable de l'élevage (PNDEL) adoptée en 2010 sert de cadre de référence pour le secteur de l'élevage au Burkina Faso ; son Plan d'action et son Programme d'investissement pour le sous-secteur de l'élevage (PAPISE) décrivent les opérations et ont à leur tour alimenté les programmes axés sur l'élevage du Programme national du secteur rural (PNSR).

\section{PNSR (I et II) : Programmes et évaluation des infrastructures d'élevage}


Dans la première phase du PNSR couvrant la période 2011-2015, plusieurs programmes se sont concentrés sur le secteur de I'élevage, dont trois concernent les infrastructures (voir Tableau 4.4.) :

- Le sous-programme 2 de l'axe 1 visait à améliorer la productivité et la compétitivité de la production animale par la construction ou la restauration de zones d'abattage, de marchés aux bestiaux et d'abattoirs. Ce sous-programme n'a pas ciblé de régions prioritaires.

Toutefois, malgré les investissements, la production animale a diminué en 2011-2015, les exportations de bovins et de petits ruminants ayant baissé de $3 \%$ et $4 \%$ respectivement (MRAH, 2018).

- Le sous-programme 3 de l'axe 3 sur la sécurisation et la gestion des ressources pastorales (dans les actions 2 et 3 ) prévoyait de faire passer le nombre de zones pastorales de 25 à 40 en 2015 et d'assurer leur fonctionnalité grâce à des infrastructures adaptées et opérationnelles (parcs de vaccination, magasins d'intrants, bureaux et logements). L'action 4 sur la promotion de l'hydraulique pastorale a ciblé les zones les plus défavorisées (un barrage, deux boulis, 15 forages et 10 puits pastoraux par an), réhabilitant les ouvrages existants et créant une base de données sur l'hydraulique pastorale. L'évaluation des infrastructures d'élevage a montré que la couverture des besoins en eau du bétail pendant la saison sèche est passée de $50 \%$ en 2011 à $61 \%$ en 2015, pour un objectif fixé à 70\%. Ceci a été rendu possible par l'exploitation de 529 forages pastoraux, 136 boulis, 130 puits de grand diamètre et 23 barrages pastoraux. En outre, de nombreux efforts ont permis d'assurer la sécurité foncière et la gestion des ressources naturelles. L'évaluation montre les réalisations suivantes : (i) la délimitation de 27 zones de pâturage et l'établissement de $1000 \mathrm{~km}$ de pistes pour le bétail ; (ii) la sécurisation de 200000 ha de pâturages ; (iii) la restauration de 10314 ha de terres dégradées ; (iv) l'amélioration de 26 zones pastorales couvrant 775000 ha (MRAH, 2018).

- Le sous-programme 3 de l'axe 1 sur l'amélioration de la santé publique animale et vétérinaire vise à renforcer le cadre juridique, la capacité de diagnostic et le système d'inspection aux frontières, dans les abattoirs, les laiteries et les lieux de vente. Toutefois, le sous-programme ne prévoyait pas la construction d'infrastructures sanitaires pour le bétail, ni ne ciblait des régions spécifiques.

Dans l'ensemble, le PNSR I a eu des effets multiples sur le secteur de l'élevage, mais n'a guère prêté attention aux emplacements possibles pour la distribution des infrastructures sur l'ensemble du territoire.

\section{Tableau 4.4. Programmes d'infrastructures et d'évaluation du bétail PNSR I, Burkina Faso}

\begin{tabular}{l|l} 
Catégories & PNSR I (2011-2015)
\end{tabular}

d'infrastructures

1 Infrastructures de production et de vente de viande
Axe 1: Sous-programme 2 - Améliorer la productivité et la compétitivité de la production animale Objectif : améliorer la compétitivité de l'élevage grâce a une intensification progressive et un meilleur accès aux marchés pour les éleveurs.
Actions : (i) construire des infrastructures (une zone d'abattage par commune, des marchés à bétail, cinq abattoirs, etc.) ; (ii) réhabiliter les infrastructures existantes ; (iii) équiper les infrastructures ; (iv) mettre en place des organes de gestion et d'exploitation ; et ( $v$ ) former les acteurs de ces organes.
$\rightarrow$ Pas de détails sur la dimension spatiale

2 Infrastructures d'abreuvement et de pâturage

\section{Axe 3: Sous-programme 3 - Sécuriser et gérer les ressources pastorales}

- Objectifs : (i) renforcer le cadre juridique et réglementaire pour sécuriser les activités d'élevage ; (ii) sécuriser et rendre fonctionnelles les zones pastorales ; (iii) assurer un accès durable à l'eau pour les animaux et les activités de production animale ; et (iv) mettre en place des mécanismes de prévention et de gestion des crises du bétail et des vulnérabilités alimentaires.

\section{$\rightarrow$ Pas de détails sur le choix des zones prioritaires}

3 Infrastructures de santé

\section{Axe 1: Sous-programme 3 - Améliorer la santé publique animale et vétérinaire}

- Objectif : «Prévention, contrôle et éradication efficaces des maladies animales prioritaires ».

Aucune infrastructure de santé et aucune considération de la dimension spatiale

Source : Les Auteurs, sur la base des données du MRAH (2011).

La deuxième phase du PNSR (PNSR II) s'aligne sur les programmes nationaux tels que la stratégie de développement rural (SDR) et le plan national de développement économique et social (PNDES), en fournissant des infrastructures supplémentaires pour le bétail, telles que des infrastructures sanitaires, avec la création d'une centrale d'achat de médicaments vétérinaires (CAMVET) et d'unités de production de vaccins. Toutefois, le programme a une portée générale et ne cible pas des zones spécifiques. Les objectifs de productivité et de compétitivité du PNSR II concernent les abattoirs réfrigérés de Ouagadougou et Bobo-Dioulasso, qui faciliteront les exportations de viande, mais nécessitent des mesures supplémentaires pour répondre aux normes internationales requises (MRAH, 2010). Le sous-programme de la phase II sur la sécurisation et la gestion des ressources pastorales ne prévoit aucun ciblage géographique. 


\section{Répartition optimale des investissements sur le plan géographique}

Le PNSR II accorde peu d'attention à la hiérarchisation des zones géographiques. Les cartes sur l'adéquation relative des infrastructures d'élevage et sur la distribution des ruminants au Burkina Faso fournissent des informations clés pour mieux orienter la politique d'investissement et réduire les disparités spatiales. Le Tableau 4.5. présente chaque catégorie d'infrastructure, en indiquant les provinces les plus sous-dotées (c'est-à-dire avec un indice d'adéquation relative inférieur à $30 \%$ ). La dernière colonne résume les priorités correspondantes dans le PNSR II, tout en reliant cette analyse aux priorités d'investissement du gouvernement pour le secteur de l'élevage.

\begin{tabular}{|c|c|c|c|}
\hline & $\begin{array}{l}\text { Catégories d'in- } \\
\text { frastructure }\end{array}$ & $\begin{array}{l}\text { Provinces les plus } \\
\text { sous-dotées } \\
(\text { IAR }<=30 \%)\end{array}$ & PNSR II (2016-2020) \\
\hline 1 & $\begin{array}{l}\text { Infrastructures } \\
\text { de pâturage }\end{array}$ & $\begin{array}{l}\text { Kouritenga, Sanma- } \\
\text { tenga, Sissili, Ziro, } \\
\text { Bazega, Kadiogo, } \\
\text { Houet, Tuy, Ganzour- } \\
\text { gou, Poni }\end{array}$ & \multirow[t]{2}{*}{$\begin{array}{l}\text { Axe 3: Sous-programme « Sécurité et gestion durable des ressources pastorales » } \\
\text { - Promotion et gestion de l'hydraulique pastorale à travers la création de points d'eau } \\
\text { (forages, boulis, barrages et puits pastoraux) et d'une base de données. } \\
\text { - Rendre les zones pastorales fonctionnelles en les dotant d'infrastructures adaptées } \\
\text { (points et plans d'eau, magasins d'intrants, parcs de vaccination, etc.) } \\
\quad \rightarrow \text { Pas de détails sur la dimension spatiale }\end{array}$} \\
\hline 2 & $\begin{array}{l}\text { Infrastructures } \\
\text { d'abreuvement }\end{array}$ & $\begin{array}{l}\text { Gnagna, Tapoa, Ou- } \\
\text { dalan, Yagha }\end{array}$ & \\
\hline 3 & $\begin{array}{l}\text { Infrastructures } \\
\text { de santé }\end{array}$ & $\begin{array}{l}\text { Kouritenga, } \\
\text { Sanmatenga, } \\
\text { Gnagna, Tapoa, } \\
\text { Yatenga }\end{array}$ & $\begin{array}{l}\text { Axe 1: Sous-programme « santé publique animale et vétérinaire » } \\
\text { - Objectif du programme : «Prévention, contrôle et éradication efficaces des maladies } \\
\text { animales prioritaires et des maladies transfrontalières ». } \\
\text { - Des constructions centralisées : (i) Office central d'achat de médicaments vétéri- } \\
\text { naires (CAMVET) ; (ii) unité de production de vaccins. } \\
\quad \rightarrow \text { Précision spatiale incertaine }\end{array}$ \\
\hline 4 & $\begin{array}{l}\text { Infrastructures } \\
\text { de vente }\end{array}$ & $\begin{array}{l}\text { Mouhoun, Namen- } \\
\text { tenga, Boulkiemde, } \\
\text { Seno }\end{array}$ & \multirow{3}{*}{$\begin{array}{l}\text { Axe 2: Sous-programme «Productivité et compétitivité de l'élevage » (principale- } \\
\text { ment l'action 5) } \\
\text { - Développement d'une stratégie nationale pour les fournitures d'intrants et les équi- } \\
\text { pements pour le bétail. } \\
\text { - Construction : (i) abattoirs réfrigérés à Bobo-Dioulasso (Houet) et dans cinq autres } \\
\text { localités; (ii) laiteries dans chaque bassin laitier à Bobo-Dioulasso (Houet) et à à } \\
\text { Ouagadougou (Kadiogo) } \\
\rightarrow \text { Objectifs moins prioritaires }\end{array}$} \\
\hline 5 & $\begin{array}{l}\text { Infrastructures } \\
\text { de production de } \\
\text { viande }\end{array}$ & $\begin{array}{l}\text { Sanmatenga, } \\
\text { Gnagna, Tapoa, } \\
\text { Oudalan, Seno }\end{array}$ & \\
\hline 6 & $\begin{array}{l}\text { Infrastructures } \\
\text { de production de } \\
\text { lait }\end{array}$ & $\begin{array}{l}\text { Sanmatenga, Ouda- } \\
\text { Ian, Seno, Soum }\end{array}$ & \\
\hline
\end{tabular}

Source : Les Auteurs, sur la base des données du MRAH $(2016,2018)$, DGEAP/MRAH (2009). 


\section{NOTE DE PLAIDOYER DU CHAPITRE 4}

Messages clés tirés de ce chapitre :

1. Le sous-secteur de l'élevage présente de multiples avantages, tels qu'un régime alimentaire plus nutritif et plus diversifié, des services de labourage et d'assurance, et la production de fumier.

2. Afin d'accroître la productivité et de répondre à la demande croissante de produits alimentaires d'origine animale, il est important d'investir dans le sous-secteur de l'élevage.

3. L'attention portée à la dimension spatiale (la répartition géographique du bétail et des infrastructures d'élevage) permettra de mieux cibler les investissements et de mieux exploiter le potentiel réel de production animale du pays.

4. En utilisant un indice d'adéquation relative basé sur le nombre de têtes de bétail et d'infrastructures d'élevage, on observe une disparité entre le Nord-Est et le Sud-Ouest du Burkina Faso. Les provinces du Nord-Est, bien que abondamment peuplées de ruminants, sont relativement dépourvues d'infrastructures d'abreuvement, de santé, de production de viande et de lait. Les cartes d'adéquation relative des infrastructures de pâturage et de vente décrivent une autre situation, avec des résultats plus mitigés.

5. Les zones les plus déficientes, par type d'infrastructure, sont résumées dans le tableau ci-dessous.

\section{\begin{tabular}{l|l} 
Types d'infrastructure & Provinces les plus sous-dotées
\end{tabular}}

\begin{tabular}{|l|l|}
\hline Pâturage & $\begin{array}{l}\text { Kouritenga, Sanmatenga, Sissili, Ziro, Bazega, Kadiogo, Houet, Tuy, } \\
\text { Ganzourgou, Poni }\end{array}$ \\
\hline Abreuvement & Gnagna, Tapoa, Oudalan, Yagha \\
\hline Santé & Kouritenga, Sanmatenga, Gnagna, Tapoa, Yatenga \\
\hline Vente & Mouhoun, Namentenga, Boulkiemde, Seno \\
\hline Production de viande & Sanmatenga, Gnagna, Tapoa, Oudalan, Seno \\
\hline Production de lait & Sanmatenga, Oudalan, Seno, Soum \\
\hline
\end{tabular}

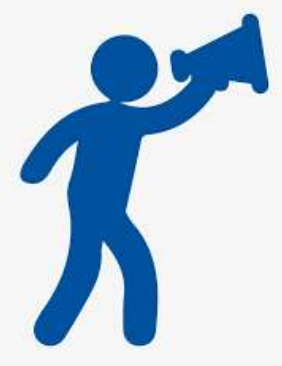




\section{Chapitre 5}

\section{LES OBSTACLES ET LES SOLUTIONS DE PLAIDOYER EN FONCTION DES ZONES GÉOGRA- PHIQUES}

Le principe de base de cet atlas politique est que la transformation agricole se fait dans un contexte hétérogène. En étant sensible à la géographie et en tenant compte des variations biophysiques, économiques et sociales à travers le pays, la conception et la mise en œuvre des politiques pourraient être plus efficaces. Si ce point de vue est devenu partie intégrante du discours dominant sur le développement depuis plusieurs décennies, les outils capables de gérer l'hétérogénéité sont restés rares. Dans cet atlas politique, nous nous appuyons sur des cartes pour mettre en évidence et décrire l'hétérogénéité, identifier les obstacles et suggérer des solutions de plaidoyer spécifiques à chaque zone.

Les cartes sont des outils puissants pour saisir I'hétérogénéité, établir des priorités et développer une stratégie de plaidoyer. En désagrégeant les données par unités administratives d'un pays (c'est-à-dire les régions et les provinces), des données de différentes natures telles que la production alimentaire, la consommation ou les données climatiques peuvent être combinées dans une même analyse - même si elles ont été mesurées de manière très différente. Les cartes permettent de résumer les données et les résultats dans une image visuelle concise, contrairement à un tableau qui nécessiterait de nombreuses lignes (une pour chaque région ou province) et qui serait peu attrayant et inutile pour afficher des relations spatiales. Enfin, les cartes peuvent raconter une histoire convaincante instantanément, ce qui est une caractéristique importante lorsque l'on a affaire à des décideurs politiques pressés par le temps.

Mais quelle est I'histoire racontée par cet atlas politique?

La faim cachée est un problème sérieux dans la plupart des régions du pays. Même si les ménages couvrent (en moyenne) $80 \%$ de leur apport énergétique et protéique recommandé, les adéquations des micronutriments sont nettement inférieures. La vitamine B12, le calcium et la vitamine A sont les plus problématiques, avec des taux d'adéquation nationaux allant de $27,9 \%$ à $35,4 \%$. Les apports en fer, en zinc et en folate sont plus élevés et se situent entre $62,5 \%$ et $79,1 \%$. Étant donné qu'une consommation adéquate de micronutriments permet d'obtenir de meilleurs résultats en matière de nutrition, la lutte contre la faim cachée pourrait être un sujet de plaidoyer important pour les OSC. Comme de nombreux donateurs et agences de développement exigent aujourd'hui que les interventions soient « sensibles à la nutrition », les OSC pourraient contribuer à harmoniser les efforts des gouvernements tout au long du système alimentaire, de la production à la consommation finale.

Comme cela a été mentionné dans l'introduction, les OSC travaillant sur la SAN dans le cadre du programme V4CP s'attachent à supprimer les contraintes de production grâce à la modernisation des exploitations familiales. Bien que les inefficacités de la production agricole soient omniprésentes dans le pays, les résultats du chapitre 2 montrent que Comoe, Tapoa, Gourma, Seno et Yagha souffrent à la fois d'une efficacité de production inférieure à la moyenne et de taux de retard de croissance plus élevés. Si d'autres provinces peuvent également souffrir d'une faible efficacité de production, l'effet sur les résultats nutritionnels finaux est plus faible. Dans le même ordre d'idées, d'autres provinces prioritaires pourraient également bénéficier d'une plus grande efficacité de production, mais d'autres obstacles semblent plus critiques.

Selon l'analyse de l'adéquation des nutriments présentée au chapitre 3, la production de vitamine B12 et de vitamine A est très faible au Burkina Faso, ce qui se traduit par des taux d'apport faibles. La réduction des contraintes de production d'aliments contenant ces deux micronutriments pourrait contribuer à augmenter l'apport en micronutriments. Pour la vitamine A, que I'on trouve dans divers aliments horticoles, des conditions agroécologiques appropriées sont essentielles - comme les précipitations plus régulières et plus abondantes dans les provinces du Sud.

Comme la vitamine B12 se trouve exclusivement dans les aliments d'origine animale, I'augmentation de la production de viande et de produits laitiers se fait en fonction des infrastructures d'élevage disponibles. L'analyse spatiale du chapitre 4 a révélé la sous-dotation relative des différents types d'infrastructures d'élevage pour faire face aux concentrations importantes de grands et petits ruminants dans le Nord-Est. A l'exception du Gourma, cette observation vaut pour Seno, Yagha et Tapoa et concerne principalement les infrastructures d'abreuvement, de santé et de production de viande.

Les contraintes d'accès semblent plus critiques pour les provinces prioritaires de Kourweogo, Tuy, Bougouriba et Ziro. La distribution de céréales et de légumineuses - actuellement les sources les plus importantes de fer, de zinc et de folate - souffre de « pertes de nutriments », ce qui entraîne des carences en micronutriments. À l'exception de la région Centre, la production de ces trois minéraux dépasse la quantité disponible sur les marchés locaux. En raison des différences agroécologiques entre les 
provinces du Nord et du Sud du Burkina Faso, les sites de production d'aliments riches en vitamine A et les centres de consommation devraient être bien reliés à travers le pays avec des infrastructures de transport, de stockage et de traitement appropriées. En ce qui concerne le calcium, la consommation des ménages pourrait augmenter considérablement si une plus grande partie de la production de sésame du Burkina Faso était transformée pour les marchés intérieurs. Actuellement, la plupart de ces graines riches en calcium sont exportées vers des pays étrangers, ce qui en fait la deuxième culture d'exportation du Burkina Faso.

Les contraintes d'utilisation semblent critiques pour la plupart des provinces souffrant d'un taux élevé de retard de croissance chez les enfants, en particulier Gourma, Seno, Tapoa, Yagha, Comoe, Bougouriba, Ziro, Loroum, Yatenga, Oudalan, Soum et Ioba. Dans ces provinces, l'accès à une gamme variée de produits alimentaires est moins important que la manière dont les aliments sont utilisés pour obtenir des résultats nutritionnels. Outre les pratiques culinaires et la répartition intra-ménage, cette conversion dépend des infrastructures et des pratiques en matière de santé, d'eau et d'assainissement.

Tous les ciblages ne devraient pas être déterminés par la situation géographique. Un grand nombre de personnes défavorisées et mal nourries peuvent vivre dans des zones administratives dont les performances moyennes semblent moins critiques, en raison d'une inégalité interne prononcée au sein de ces zones. Les obstacles de la SAN devront être surmontés avec le temps. À court terme, les programmes de protection sociale pourraient garantir un apport minimal de nutriments essentiels aux ménages les plus sous-alimentés. Ces programmes peuvent prendre la forme de transferts de nourriture ou d'argent, d'enrichissement des aliments ou de supplémentation en nutriments. La capitale (et la région du centre qui l'entoure) présente de faibles scores de consommation alimentaire et de faibles taux d'adéquation au niveau des ménages pour presque tous les nutriments considérés dans cette analyse. Compte tenu de sa forte population, l'introduction réussie d'une protection sociale tenant compte de la nutrition à Ouagadougou pourrait améliorer considérablement la SAN dans le pays.

Diverses politiques et programmes clés sur le developpement de l'agriculture, de la nutrition et du secteur de l'élevage pourraient être enrichis avec un ciblage géographique. Les cartes de cet atlas politique pourraient ainsi aider les OSC à donner la priorité à certains sujets de plaidoyer ainsi que leurs stratégies correspondantes pour orienter les politiques sur la meilleure façon de traiter les obstacles spécifiques à la géographie du pays. 
Brown de Colstoun, E. C., Huang, C., Wang, P., Tilton, J. C., Tan, B., Phillips, J., ... Wolfe., R. E. (2017). Global Man-made Impervious Surface (GMIS) Dataset From Landsat. Palisades, NY: NASA Socioeconomic Data and Applications Center (SEDAC). https://doi.org/https://doi.org/10.7927/H4P55KKF

Ericksen, P. J. (2008). Conceptualizing food systems for global environmental change research. Global Environmental Change, 18, 234245. https://doi.org/10.1016/j.gloenvcha.2007.09.002

FAO. (2011). Guidelines for the preparation of livestock sector reviews. Animal Production and Health Guidelines. No. 5. Rome: Food and Agriculture Organization of the United Nations.

FAO. (2019). Le devenir de l'élevage au Burkina Faso. Défis et opportunités face aux incertitudes. Rome: Organisation des Nations unies pour l'alimentation et I'agriculture. https://doi.org/10.1007/978-94-007-2512-6_8

Gerber, P., Mooney, H. A., Dijkman, J., Tarawali, S., \& de Haan, C. (2010). Livestock in a Changing Landscape: Experiences and Regional Perspectives. Washington/Covelo/London: Island Press.

Gillespie, S., \& van den Bold, M. (2017). Agriculture, Food Systems, and Nutrition: Meeting the Challenge. Global Challenges, 1(3), 112. https://doi.org/10.1002/gch2.201600002

Glin, L. C., Mol, A. P. J., \& Oosterveer, P. (2013). Conventionalization of the organic sesame network from Burkina Faso: shrinking into mainstream. Agriculture and Human Values, 30(4), 539-554. https://doi.org/10.1007/s10460-013-9435-9

Global Panel on Agriculture and Food Systems for Nutrition. (2016). Food systems and diets: Facing the challenges of the 21st century. London. https://doi.org/http://glopan.org/sites/default/files/ForesightReport.pdf

Hansen, M. C., Potapov, P. V., Moore, R., Hancher, M., Turubanova, S. A., Tyukavina, A., ... Townshend, J. R. G. (2013). HighResolution Global Maps of 21st-Century Forest Cover Change. Science, 342(November), 850-853. https://doi.org/10.1126/science. 1244693

Institut National de la Statistique et de la Démographie du Burkina Faso. (2016). Burkina Faso - Enquête Multisectorielle Continue 2014. Ouagadougou: Institut National de la Statistique et de la Démographie; Ministère de l'Economie et des Finances.

Jones, A. D., \& Ejeta, G. (2015). A new global agenda for nutrition and health: the importance of agriculture and food systems. Bulletin World Health Organization, 94(December), 228-229. https://doi.org/10.2471/BLT.15.164509

Ministère de l'Agriculture du Burkina Faso. (2010). Etude sur la dynamique de la consommation alimentaire au Burkina Faso. Ouagadougou: Direction Générale de la Promotion de L'Economie Rurale (DGPER), Direction de la Prospective et des Statistiques Agricoles et Alimentaires (DPSAA).

Ministère de l'Agriculture du Burkina Faso. (2015). Résultats Définitifs de la Campagne Agricole 2014/2015 et Perspectives de la Situation Alimentaire et Nutritionnelle. Ouagadougou: Secrétariat General, Direction Générale des Etudes et des Statistiques Sectorielles.

Ministère de l'Agriculture du Burkina Faso. (2016). Résultats Définitifs de la Campagne Agricole 2015/2016 et Perspectives de la Situation Alimentaire et Nutritionnelle. Ouagadougou: Secrétariat General, Direction Générale des Etudes et des Statistiques Sectorielles.

Ministère de l'Agriculture et de la Securite Alimentaire du Burkina Faso. (2014). Annuaire des Statistiques Agricoles 2012. Ouagadougou: Secrétariat General, Direction Générale des Etudes et des Statistiques Sectorielles.

Ministère de la Sante du Burkina Faso. (2016). Enquête Nutritionnelle Nationale 2016, Rapport Finale. Ouagadougou: Direction de la Nutrition.

Ministère des Ressources Animales du Burkina Faso. (2015). Annuaires des Statistiques de I'Elevage 2014. Ouagadougou: Secrétariat General, Direction Générale des Etudes et des Statistiques Sectorielles.

MRAH. (2011). Programme National du Secteur Rural (2011-2015) du Burkina Faso. Ouagadougou: Ministère des Ressources Animales et Halieutiques.

MRAH. (2018). Deuxième Programme National du Secteur Rural 2016-2020. Ouagadougou: Ministère des Ressources Animales et Halieutiques.

Ollo, S., Ouedraogo, T., Degueurse, S., Ouattara, P., \& Hitayezu, J.-D. (2013). Burkina Faso: Evaluation Approfondie sur la Sécurité Alimentaire en Situation d'Urgence dans les Camps de Refugiés Maliens et Villages Hôtes. Rome: Programme Alimentaire Mondial, Service de I'Analyse de la Sécurité Alimentaire (VAM).

Ouedraogo, I., Tigabu, M., Savadogo, P., Compaore, H., Oden, P. C., \& Ouadba, J. M. (2010). Land Cover Change and Its Relation With Population Dynamics in Burkina Faso, West Africa. Land Degradation and Development, 21(5), 453-462.

Pangaribowo, E. H., Gerber, N., \& Torero, M. (2013). Food and nutrition security indicators: A review (ZEF Working Paper No. 108). Bonn. https://doi.org/ISSN 1864-6638

Pekel, J.-F., Cottam, A., Gorelick, N., \& Belward, A. S. (2016). High-resolution mapping of global surface water and its long-term changes. Nature, 540(418-422). https://doi.org/https://doi.org/10.1038/nature20584

Pica-Ciamarra, U., Baker, D., Morgan, N., Zezza, A., Azzarri, C., Ly, C., ... Sserugga, J. (2016). Investir dans le secteur de l'élevage; l'importance de chiffres précis. Washington, DC: Banque internationale pour la reconstruction et le développement / Banque mondiale.

Pinstrup-Andersen, P. (2013). Nutrition-sensitive food systems: From rhetoric to action. The Lancet, 382, $375-376$. https://doi.org/10.1016/S0140-6736(13)61053-3

SP/CPSA. (2017). Deuxième Programme National du Secteur Rural (PNSR) 2016-2020. Ouagadougou: Secrétariat Permanent de la Coordination des Politiques Sectorielles Agricoles.

Stadlmayr, B., Charrondiere, R. U., Enujiugha, V. N., Bayili, R. G., Fagbohoun, E. G., Samb, B., ... Burlingame, B. (2012). West African Food Composition Table. Rome: Food and Agricultural Organization of the United Nations. Retrieved from http://www.fao.org/docrep/015/i2698b/i2698b00.pdf

Stephens, E. C., Jones, A. D., \& Parsons, D. (2018). Agricultural systems research and global food security in the 21st century: An overview and roadmap for future opportunities. Agricultural Systems, 163, 1-6. https://doi.org/10.1016/j.agsy.2017.01.011

Tendall, D. M., Joerin, J., Kopainsky, B., Edwards, P., Shreck, A., Le, Q. B., ... Six, J. (2015). Food system resilience: Defining the concept. Global Food Security, 6(October), 17-23. https://doi.org/10.1016/j.gfs.2015.08.001

Torero, M. (2014). Targeting investments to link farmers to markets: a framework for capturing the heterogeneity of smallholder 
farmers. In P. B. R. Hazell \& A. Rahman (Eds.), New Directions for Smallholder Agriculture. Oxford: Oxford University Press. https://doi.org/10.1093/acprof

UNEP-WCMC. (2018). Protected Area Profile for Burkina Faso from the World Database of Protected Areas. Retrieved from www.protectedplanet.net

WFP. (2008). Food Consumption Analysis, Calculation and use of the food consumption score in food security analysis. Rome: World Food Programme, Vulnerability Analysis and Mapping Branch (ODAV).

Xiong, J., Thenkabail, P. S., Tilton, J. C., Gumma, M. K., Teluguntla, P., Oliphant, A., ... Gorelick, N. (2017). Nominal 30-m cropland extent map of continental Africa by integrating pixel-based and object-based algorithms using Sentinel-2 and Landsat-8 data on Google Earth Engine. Remote Sensing, 9(10), 1-27. https://doi.org/10.3390/rs9101065 

Le système régional d'analyse stratégique et de gestion des connaissances (ReSAKSS) contribue à réussite de la mise en œuvre du Programme Détaillé de Développement de l'Agriculture en Afrique (PDDAA) en fournissant des données pertinentes pour l'élaboration des politiques, en facilitant le dialogue entre les parties prenantes, en suivant les progrès accomplis dans la revue des objectifs et en renforçant les processus de responsabilisation mutuelle aux niveaux continental, régional et national.

Les atlas nationaux du ReSAKSS (RCeA) sont des outils de cartographie basés sur les SIG et conçus pour aider les analystes et les décideurs politiques à accéder à des données de grande qualité hautement désagrégées sur les indicateurs agricoles, socio-économiques et bio-physiques et à les utiliser pour orienter les décisions en matière de politique agricole et d'investissement.
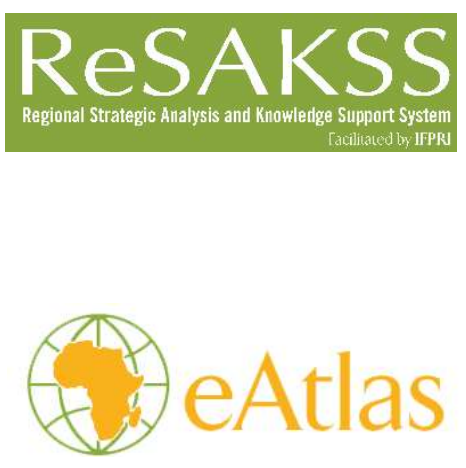

\section{A propos des auteurs}

Wim Marivoet, Chercheur, John M. Ulimwengu, Chercheur senior, et Leysa Maty Sall, Analyste de recherche au Bureau Régional pour l'Afrique de I'Ouest et du Centre de l'Institut international de recherche sur les politiques alimentaires (IFPRI). Cet atlas politique a été préparé en étroite collaboration avec I'équipe technique des atlas nationaux du ReSAKSS dirigée par Mohamed Abd Salam El Vilaly et Racine Ly, et avec Maurice Taondyande (ReSAKSS-AO). Les auteurs tiennent à remercier les nombreux participants qui ont contribué à la discussion lors de divers ateliers nationaux (2018-2020). Ils sont également reconnaissants à Fernando Sedano, Mariam Katile, Jean Hubert Zongo, Desiré Nanema, Issaka Tingri, Yassia Kindo et Abdelaziz Ouedraogo pour leur aide dans l'analyse des données et leurs commentaires sur les différents chapitres, ainsi qu'à Julie Kurtz, Vanessa Ndong et Fleur Wouterse pour la révision, la traduction et la révision finale.

\section{INSTITUT INTERNATIONAL DE RECHERCHE SUR LES POLITIQUES ALIMENTAIRES \\ Membre du Consortium CGIAR | Un monde sans faim ni malnutrition \\ Bureau Afrique de l'Ouest et du Centre | Lot \#2 Titre 3396 - BP 24063, Dakar-Almadies, Sénégal \\ Tél. : +221338699800 | Fax : +221338699841 \\ Email : ifpri-dakar@cgiar.org | wca.ifpri.info}

